\title{
Exploring deep brain stimulation as a treatment for tinnitus
}

Citation for published version (APA):

Smit, J. V. (2018). Exploring deep brain stimulation as a treatment for tinnitus. [Doctoral Thesis, Maastricht University]. Maastricht University. https://doi.org/10.26481/dis.20180921js

Document status and date:

Published: 01/01/2018

DOI:

10.26481/dis.20180921js

Document Version:

Publisher's PDF, also known as Version of record

\section{Please check the document version of this publication:}

- A submitted manuscript is the version of the article upon submission and before peer-review. There can be important differences between the submitted version and the official published version of record.

People interested in the research are advised to contact the author for the final version of the publication, or visit the DOI to the publisher's website.

- The final author version and the galley proof are versions of the publication after peer review.

- The final published version features the final layout of the paper including the volume, issue and page numbers.

Link to publication

\footnotetext{
General rights rights.

- You may freely distribute the URL identifying the publication in the public portal. please follow below link for the End User Agreement:

www.umlib.nl/taverne-license

Take down policy

If you believe that this document breaches copyright please contact us at:

repository@maastrichtuniversity.nl

providing details and we will investigate your claim.
}

Copyright and moral rights for the publications made accessible in the public portal are retained by the authors and/or other copyright owners and it is a condition of accessing publications that users recognise and abide by the legal requirements associated with these

- Users may download and print one copy of any publication from the public portal for the purpose of private study or research.

- You may not further distribute the material or use it for any profit-making activity or commercial gain

If the publication is distributed under the terms of Article $25 \mathrm{fa}$ of the Dutch Copyright Act, indicated by the "Taverne" license above, 




\section{Exploring deep brain stimulation as a treatment for tinnitus}




\section{Colofon}

Author: Jasper V. Smit

Cover: Jasper V. Smit (design), Geert Jan Zonneveld (DBS illustration)

Printing: Gildeprint - The Netherlands

ISBN: 978-94-9301-429-9

Printing of this thesis was financially supported by: Beter Horen, Cochlear, Daleco Pharma B.V., Dos Medical B.V. / kno-winkel.nl, EmiD audiologische apparatuur, Laservision, Meda Pharma B.V., MED-EL, MediTop, Oticon, Phonak, Schoonenberg, Specsavers, ZEISS.

(C) Jasper V. Smit, The Netherlands, 2018

All rights reserved. No part of this publication may be reproduced or transmitted in any form by any means, without permission of the author. 


\title{
Exploring deep brain stimulation as a treatment for tinnitus
}

\author{
PROEFSCHRIFT \\ Ter verkrijging van de graad van doctor aan de Universiteit Maastricht, \\ op gezag van de Rector Magnificus, Prof. Dr. R.M. Letschert \\ volgens het besluit van het College van Decanen, \\ in het openbaar te verdedigen op
}

vrijdag 21 september 2018 om 12:00 uur

door

Jasper V. Smit

Geboren op 6 december 1985 te Epe 


\section{Promotores}

Prof. dr. R.J. Stokroos

Prof. dr. Y. Temel

\section{Co-promotor}

Dr. A. Jahanshahianvar

\section{Beoordelingscommissie}

Prof. B. Kremer (voorzitter)

Prof. dr. P. van Dijk, Universitair Medisch Centrum Groningen

Prof. dr. B.P.F. Rutten

Dr. H. Thomeer, Universitair Medisch Centrum Utrecht

Dr. L. Ackermans 


\section{Contents}

\section{Chapter 1}

General introduction

\section{Chapter 2}

Deep brain stimulation in tinnitus: Current and future perspectives

\section{Chapter 3}

The impact of deep brain stimulation on tinnitus

\section{Chapter 4}

Patient acceptance of invasive treatments for tinnitus

\section{Chapter 5}

Deep brain stimulation of the inferior colliculus in the rodent suppresses tinnitus

87

\section{Chapter 6}

Hearing assessment during deep brain stimulation of the central nucleus of the inferior colliculus and dentate cerebellar nucleus in rat

\section{Chapter 7}

General discussion

Summary 139

Samenvatting 143

Valorisation 147

Dankwoord 151

Biography 


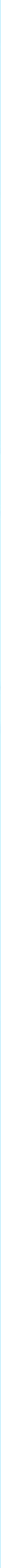




\section{Bhapter 1}

General introduction 
Tinnitus, also known as ringing in the ears, is defined as 'the conscious perception of an auditory sensation in the absence of a corresponding external stimulus' (Baguley et al., 2013). In terms of auditory abilities, tinnitus can be defined as the inability to experience silence (Deshaies et al., 2011). In The Netherlands between 1.3 and 2.0 million adults suffer from tinnitus (Davis and El Rafaie, 2000, Quaranta et al., 1996, Centraal Bureau voor de Statistiek, 2016). Though tinnitus is a debilitating phenomenon, the burden widely varies amongst patients. For example, the least severe degree of tinnitus ('tinnitus does not bother me') is reported to be experienced in $24 \%$ of the tinnitus patients, while the most severe degree of tinnitus ('tinnitus plaques me all day') is experienced in $17 \%$ (Axelsson and Ringdahl, 1989). Especially for patients with this severe degree of tinnitus, close correlations with psychological morbidity are present, such as anxiety, depression and sleep disorders (Langguth, 2011, Zoger et al., 2006). In a few rare cases tinnitus even drives patients to commit suicide (Pridmore et al., 2012).

Besides affecting quality of life, tinnitus has great impact on society. The mean annual health care costs of tinnitus are estimated to be $€ 1544$ per patient (Maes et al., 2013). Despite these high costs for society there is limited funding for research, for example only $2 \%$ of the hearing research funds of the US National Institutes of Health is allocated to tinnitus (Cederroth et al., 2013).

Clinically effective treatments for tinnitus are scarce. In few cases, tinnitus is a symptom of a specific pathology, such as a cerebellopontine tumor or neurovascular conflict, which can be treated with otoneurosurgery (De Ridder et al., 2007). If tinnitus and hearing loss are symptoms of middle ear pathology (e.g. otospongiosis), conductive hearing loss can permanently be restored by means of surgery (e.g. stapedotomy) thereby improving tinnitus (Chang and Cheung, 2014, Ayache et al., 2003).

Unfortunately, in most cases tinnitus is accompanied by irreversible sensorineural hearing loss, induced by for example noise trauma. If hearing loss is mild to severe, a hearing aid is usually the first step in clinical care. There is however only limited evidence for hearing aids as a treatment for tinnitus (Hoare et al., 2014). Hearing care professionals estimate that only one fifth of the patients have a clinically relevant reduction of tinnitus by the use of hearing aids (Kochkin and Tyler, 2008). Coping strategy therapies based on cognitive-behaviour or habituation have been developed for patients with high psychological burden of tinnitus (Zachriat and Kroner-Herwig, 2004, Jastreboff, 1999). A mean reduction around one third in tinnitus impairment and its severity has recently been demonstrated (Cima et al., 2012). These therapies might reduce the burden that is accompanied by tinnitus, but are not effective in all patients and are not able to diminish the actual tinnitus sound. Currently, no other evidence-based and effective clinical treatments are available. 


\section{Auditory pathway}

The auditory pathway is the neuronal circuit, which ascends from the eighth cranial nerve to the auditory cortex. The auditory pathway comprises several brain structures and can be divided into a classical and non-classical pathway (Figure 1).

In the classical pathway, axons from the auditory part of the eighth cranial nerve terminate in the ipsilateral cochlear nuclei. The cochlear nucleus projects to the central nucleus of the inferior colliculus. These projections involve direct connections to the central nucleus of the inferior colliculus as well as indirect connections via the superior olivary complex. These connections are mainly crossed; but also ipsilateral projections to the central nucleus of the inferior colliculus exist. The cells in the central nucleus of the inferior colliculus project to the ventral part of the medial geniculate body in the auditory thalamus. The medial geniculate body projects to the primary auditory cortex (Møller, 2011).

The ipsi- and contralateral projections from the cochlear nuclei to the central nucleus of the inferior colliculus result in bilateral representation of sound at the cortex (Møller, 2011). This reflects on the notion that auditory dysfunction is rarely seen in unilateral stroke patients (Hausler and Levine, 2000). In the non-classical pathway, the central nucleus of the inferior colliculus is connected to the external and dorsal cortex of the inferior colliculus. Neurons in the external and dorsal nucleus of the inferior colliculus project to medial and dorsal parts of the medial geniculate body. The classical pathway projects to the primary auditory cortex, whereas the non-classical pathway projects directly to the secondary and association auditory cortices. Additionally, the cells project from the medial and dorsal parts of the auditory thalamus directly and indirectly to limbic structures such as the amygdala and hippocampus (Møller, 2011).

Whilst this thesis is focused on the ascending auditory pathways, it must be noted that descending auditory pathways exist as well. Several auditory regions project descending fibers to more caudal situated auditory structures and ultimately descending fibers can influence the mechanical properties of the basilar membrane (Møller, 2011). 


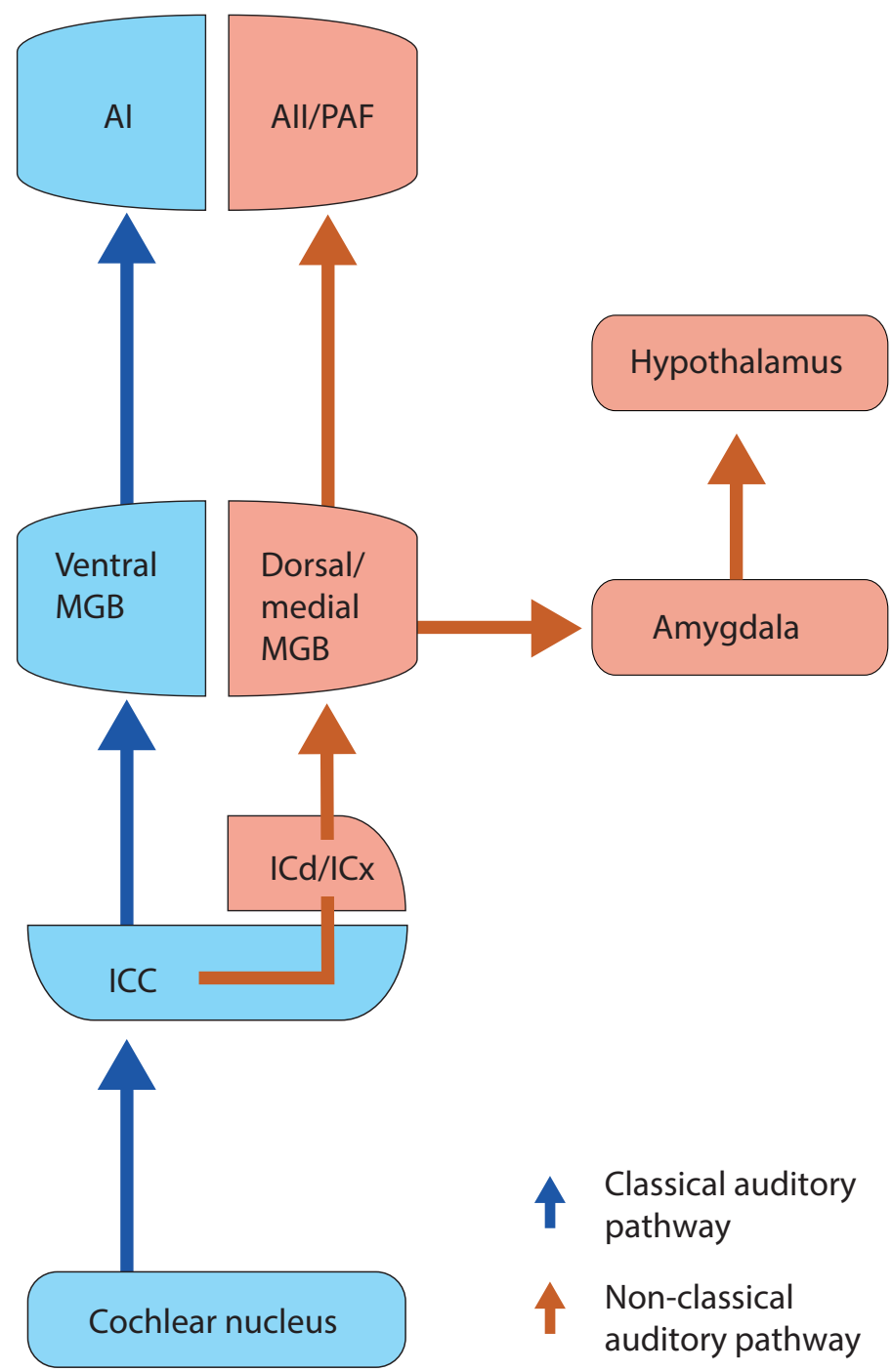

Figure 1: Classical and non-classical auditory pathway. $A I=$ primary auditory cortex, All = secondary auditory cortex, ICC, ICd and ICX are respectively the central, dorsal and external nucleus of the inferior colliculus, MGB= medial geniculate body of the thalamus, PAF = posterior auditory cortical field (Møller, 2011). 


\section{Pathophysiology of tinnitus}

For a long time it was thought that the pathophysiology of tinnitus was purely peripheral and many attempts of treatments were confined to the ear (Stephens, 1984). However, studies in the early 1980 's showed that in the majority of cases tinnitus did not improve when the eighth cranial nerve was sectioned (Barrs and Brackmann, 1984, House and Brackmann, 1981, Gardner, 1984).

It is now generally presumed that the complex-and still not fully understood - pathophysiology of tinnitus predominantly involves the central nervous system. The first step in the pathophysiology of noise-induced tinnitus is injury of inner and/or outer hair cells in the cochlea. The tonotopic defined part of the eighth cranial nerve that is related to the affected frequencies of the cochlea will in turn deafferentiate. The reduced output of the eight cranial nerve leads to an increased neuronal activity of the central auditory system and is accompanied by increased spontaneous firing rate, neural synchrony, burst activity and generation of tonotopic reorganization (Henry et al., 2014, Norena, 2011, Eggermont and Roberts, 2004, Chen et al., 2015, Kaltenbach, 2011).

In normal condition, neurons of the eighth cranial nerve activate their target neurons in the central auditory pathway. When tonotopic reorganization occurs, neurons that formerly were mostly activated by the deafferented neurons now receive input from their edge-frequency region. This altered input in the afferent auditory regions will show increased synchrony and amplified baseline spontaneous activity. This pathological neuronal activity may elicit perception of tinnitus in the auditory cortex (Norena, 2011).

Pathological neuronal activity in tinnitus might be altered by neuromodulation. This concept is based on the effect of DBS on motor symptoms in Parkinson patients. In Parkinson patients and animal models for Parkinson's disease, neurons within the motor circuitry of the basal ganglia show enhanced neuronal activity and enhanced bursty mode of firing (Bergman et al., 1994, Benazzouz et al., 2002, Janssen et al., 2012). This aberrant neuronal firing can be disrupted by high frequency stimulation (Benazzouz et al., 1995). In tinnitus also hyperactive and bursty firing neurons are present within the auditory pathway (Wu et al., 2016, Bauer et al., 2008, Kalappa et al., 2014, Kaltenbach and Zhang, 2007, Chen and Jastreboff, 1995). Given these findings, the question is raised whether or not DBS can also treat tinnitus.

\section{Deep brain stimulation}

Electrical stimulation to treat neurological diseases by modulating the nervous system has already been practiced since ancient times. The physician Scribonius Largo suggested in 46 AD the application of electric ray on the head to treat headache (Sconocchia, 1983). The electric ray is a flattened fish known 
for being capable of producing an electric discharge. Until the eighteenth century electric fishes were used to treat various neuropsychiatric disorders (Schwalb and Hamani, 2008, Kellaway, 1946).

In the 1950s the first electrodes were implanted in the brain to control pain (Heath and Mickle, 1960). The target, the septal area, was based on rat experiments (Olds and Milner, 1954, Keifer et al., 2014). Clinical success of DBS started in the late 1980s when thalamic DBS was applied in patients with Parkinson's disease and essential tremor (Benabid et al., 1987). Preclinical work on non-human primates showing that subthalamic nucleus high frequency stimulation significantly reduced Parkinsonian symptoms, was successfully translated into clinical application (Benazzouz et al., 1993). Their translational work was the basis of the treatment for over 150.000 patients with Parkinson's disease and other disorders by DBS (Wichmann and DeLong, 2016). DBS electrodes are implanted in specific brain structures during stereotaxic surgery and connected to a pulse generator, which delivers electrical current to the electrodes. The pulse generator is subcutaneously implanted under the clavicle or in the abdomen (Figure 2).

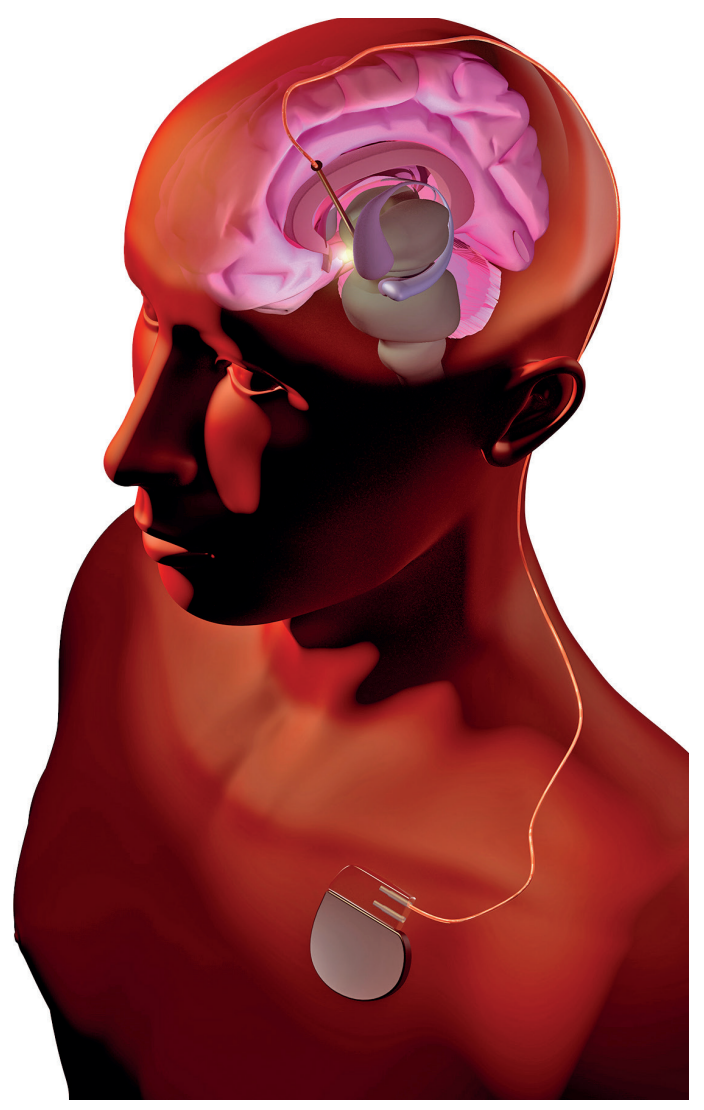

Figure 2: Illustration of a patient with deep brain stimulation. The electrode is connected with the pacemaker via a subcutaneous tunneled extension. 
The mechanism behind the therapeutic effects of DBS is still not fully understood (Hamani and Temel, 2012). It has been speculated that DBS mimics the effect of a lesion in the corresponding region (Benabid et al., 2002). One of the theories which explain this effect is that due to electrical stimulation of axon terminals a large-scale release of neurotransmitters is induced. As a result the input and output of the stimulated nucleus are dissociated and the abnormal neuronal activity is disrupted. DBS activates axonal terminals at the target, inducing extensive release of neurotransmitters, and dissociates inputs and outputs. Thus, DBS results in disruption of the abnormal information flow (Chiken and Nambu, 2016).

\section{Current problem}

Current therapies focus on psychological support and treatment of comorbidities, since no clinical effective therapy to eliminate tinnitus is available. The improved understanding of the underlying pathophysiology of tinnitus paves the way for innovative treatments. The high prevalence and corresponding socio-economic impact underlines the need for an evidence-based treatment for the severe tinnitus sufferers. The challenge is to find a therapy, which interferes directly with tinnitus sound and thereby improving quality of life.

\section{Aim of this thesis}

The overall aim of this thesis is to test the hypothesis that DBS can suppress tinnitus. To test this hypothesis, the following set of experiments has been performed.

Firstly, a review of the literature is provided to define which brain structures are potential targets for DBS in patients with tinnitus (Chapter 2). Chapter $\mathbf{3}$ presents the effect of DBS on tinnitus in patients who underwent the procedure for other indications than tinnitus. This study is performed in a multicenter setup. In Chapter $\mathbf{4}$ the results of a survey on the acceptance of invasive therapies for tinnitus patients are described. To perform DBS for tinnitus in a preclinical setting, a setup was built to induce tinnitus in rats and test them for behavioral evidence of tinnitus. In Chapter $\mathbf{5}$ the results of an animal study are reported in which DBS in the inferior colliculus was conducted. This study involves rats with behaviorally evidence of tinnitus. Chapter 6 gives more insight in hearing loss as a potential side effect of DBS. In this chapter, hearing is assessed using auditory brainstem responses in rats who are stimulated in the inferior colliculus or dentate cerebellar nucleus. Finally, an overall discussion is provided in Chapter $\mathbf{7}$ where the findings are put into scientific and clinical perspective and a synthesis is provided. 


\section{References}

Axelsson, A. \& Ringdahl, A. 1989. Tinnitus--a study of its prevalence and characteristics. Br J Audiol, 23, 53-62.

Ayache, D., Earally, F. \& Elbaz, P. 2003. Characteristics and postoperative course of tinnitus in otosclerosis. Otol Neurotol, 24, 48-51.

Baguley, D., McFerran, D. \& Hall, D. 2013. Tinnitus. Lancet, 382, 1600-7.

Barrs, D. M. \& Brackmann, D. E. 1984. Translabyrinthine nerve section: effect on tinnitus. J. Laryngol. Otol. , Suppl. 9, $287-293$.

Bauer, C. A., Turner, J. G., Caspary, D. M., Myers, K. S. \& Brozoski, T. J. 2008. Tinnitus and inferior colliculus activity in chinchillas related to three distinct patterns of cochlear trauma. J Neurosci Res, 86, 2564-78.

Benabid, A. L., Benazzous, A. \& Pollak, P. 2002. Mechanisms of deep brain stimulation. Mov Disord, 17 Suppl 3, S73-4.

Benabid, A. L., Pollak, P., Louveau, A., Henry, S. \& de Rougemont, J. 1987. Combined (thalamotomy and stimulation) stereotactic surgery of the VIM thalamic nucleus for bilateral Parkinson disease. Appl Neurophysiol, 50, 344-6.

Benazzouz, A., Breit, S., Koudsie, A., Pollak, P., Krack, P. \& Benabid, A. L. 2002. Intraoperative microrecordings of the subthalamic nucleus in Parkinson's disease. Mov Disord, 17 Suppl 3, S145-9.

Benazzouz, A., Gross, C., Feger, J., Boraud, T. \& Bioulac, B. 1993. Reversal of rigidity and improvement in motor performance by subthalamic high-frequency stimulation in MPTP-treated monkeys. Eur J Neurosci, 5, 382-9.

Benazzouz, A., Piallat, B., Pollak, P. \& Benabid, A. L. 1995. Responses of substantia nigra pars reticulata and globus pallidus complex to high frequency stimulation of the subthalamic nucleus in rats: electrophysiological data. Neurosci Lett, 189, 77-80.

Bergman, H., Wichmann, T., Karmon, B. \& DeLong, M. R. 1994. The primate subthalamic nucleus. II. Neuronal activity in the MPTP model of parkinsonism. J Neurophysiol, 72, 507-20.

Cederroth, C. R., Canlon, B. \& Langguth, B. 2013. Hearing loss and tinnitus--are funders and industry listening? Nat Biotechnol, 31, $972-4$

Chang, C. Y. \& Cheung, S. W. 2014. Tinnitus modulation by stapedectomy. Otol Neurotol, 35, 1065-9.

Chen, G. D. \& Jastreboff, P. J. 1995. Salicylate-induced abnormal activity in the inferior colliculus of rats. Hear Res, 82, 158-78.

Chen, Y. C., Li, X., Liu, L., Wang, J., Lu, C. Q., Yang, M., Jiao, Y., Zang, F. C., Radziwon, K., Chen, G. D., Sun, W., Krishnan Muthaiah, V. P., Salvi, R. \& Teng, G. J. 2015. Tinnitus and hyperacusis involve hyperactivity and enhanced connectivity in auditory-limbic-arousal-cerebellar network. Elife, 4, e06576.Chiken, S. \& Nambu, A. 2016. Mechanism of Deep Brain Stimulation: Inhibition, Excitation, or Disruption? Neuroscientist, 22, 313-22.

Cima, R. F., Maes, I. H., Joore, M. A., Scheyen, D. J., El Refaie, A., Baguley, D. M., Anteunis, L. J., van Breukelen, G. J. \& Vlaeyen, J. W. 2012. Specialised treatment based on cognitive behaviour therapy versus usual care for tinnitus: a randomised controlled trial. Lancet, 379, 1951-9.

Davis, A. \& El Rafaie, A. 2000. Epidemiology of tinnitus. In: TYLER, R. S. (ed.) Tinnitus Handbook. San Diego: Singular. 
De Ridder, D., Menovsky, T. \& Van de Heyning, P. 2007. An otoneurosurgical approach to non-pulsatile and pulsatile tinnitus. B-ENT, 3 Suppl 7, 79-86.

Deshaies, P., Gonzales, Z., Zenner, H.-P., Plontke, S., Hebert, S., Normandin, N., Girard, S.-A., Tyler, R. S. \& Cote, C. 2011. World Health Organization Regional Office for Europe: Burden of Disease from Environmental Noise: environmental noise and tinnitus Copenhagen, $W H O$.

Eggermont, J. J. \& Roberts, L. E. 2004. The neuroscience of tinnitus. Trends in Neurosciences, 27, 676-82.

Gardner, G. 1984. Neurologic surgery and tinnitus. J. Laryngol. Otol., Suppl. 9, 311-318.

Hamani, C. \& Temel, Y. 2012. Deep brain stimulation for psychiatric disease: contributions and validity of animal models. Sci Trans/ Med, 4, 142 rv8.

Hausler, R. \& Levine, R. A. 2000. Auditory dysfunction in stroke. Acta Otolaryngol, 120, 689-703.

Heath, R. G. \& Mickle, W. A. 1960. Evaluation of seven years' experience with depth electrode studies in human patients. In: RAMEY, E. R., O'DOHERTY, D.S. (ed.) Electrical studies on the unanesthetized brain. New York: Harper \& Brothers.

Henry, J. A., Roberts, L. E., Caspary, D. M., Theodoroff, S. M. \& Salvi, R. J. 2014. Underlying mechanisms of tinnitus: review and clinical implications. J Am Acad Audiol, 25, 5-22; quiz 126.

Hoare, D. J., Edmondson-Jones, M., Sereda, M., Akeroyd, M. A. \& Hall, D. 2014. Amplification with hearing aids for patients with tinnitus and co-existing hearing loss. Cochrane Database Syst Rev, CD010151.

House, J. W. \& Brackmann, D. E. 1981. Tinnitus: surgical treatment. Ciba Found Symp, 85, 204-16.

Janssen, M. L., Zwartjes, D. G., Tan, S. K., Vlamings, R., Jahanshahi, A., Heida, T., Hoogland, G., Steinbusch, H. W., Visser-Vandewalle, V. \& Temel, Y. 2012. Mild dopaminergic lesions are accompanied by robust changes in subthalamic nucleus activity. Neurosci Lett, $508,101-5$.

Jastreboff, P. J. 1999. Tinnitus retraining therapy. Br J Audiol, 33, 68-70.

Kalappa, B. I., Brozoski, T. J., Turner, J. G. \& Caspary, D. M. 2014. Single unit hyperactivity and bursting in the auditory thalamus of awake rats directly correlates with behavioural evidence of tinnitus. J Physiol, 592, 5065-78.

Kaltenbach, J. A. 2011. Tinnitus: Models and mechanisms. Hear Res, 276, 52-60.

Kaltenbach, J. A. \& Zhang, J. 2007. Intense sound-induced plasticity in the dorsal cochlear nucleus of rats: evidence for cholinergic receptor upregulation. Hear Res, 226, 232-43.

Keifer, O. P., Jr., Riley, J. P. \& Boulis, N. M. 2014. Deep brain stimulation for chronic pain: intracranial targets, clinical outcomes, and trial design considerations. Neurosurg Clin N Am, 25, 671-92.

Kellaway, P. 1946. The part played by electric fish in the early history of bioelectricity and electrotherapy. Bull Hist Med, 20, 112-37.

Kochkin, S. \& Tyler, R. S. 2008. Tinnitus treatment and the effectiveness of hearing aids: hearing care professional perceptions. Hearing Review, 15, 14-18. 
Langguth, B. 2011. A review of tinnitus symptoms beyond 'ringing in the ears': a call to action. Curr Med Res Opin, 27, 1635-43.

Maes, I. H., Cima, R. F., Vlaeyen, J. W., Anteunis, L. J. \& Joore, M. A. 2013. Tinnitus: a cost study. Ear Hear, 34, 508-14.

Møller, A. R. 2011. Anatomy and Physiology of the Auditory System. Textbook of Tinnitus.

Norena, A. J. 2011. An integrative model of tinnitus based on a central gain controlling neural sensitivity. Neurosci Biobehav Rev, 35, 1089-109.

Olds, J. \& Milner, P. 1954. Positive reinforcement produced by electrical stimulation of septal area and other regions of rat brain. $J$ Comp Physiol Psychol, 47, 419-27.

Pridmore, S., Walter, G. \& Friedland, P. 2012. Tinnitus and suicide: recent cases on the public record give cause for reconsideration. Otolaryngol Head Neck Surg, 147, 193-5.

Quaranta, A., Assennato, G. \& Sallustio, V. 1996. Epidemiology of hearing problems among adults in Italy. Scand Audiol Suppl, 42, 9-13.

Schwalb, J. M. \& Hamani, C. 2008. The history and future of deep brain stimulation. Neurotherapeutics, 5, 3-13.

Sconocchia, S. 1983. Scribonii Largi Compositiones. Teubner Akademie der Wissenschaften, Leipzig.

Statistiek, C. B. v. d. 2016. Bevolking; kerncijfers. Den Haag/Heerlen

Stephens, S. D. 1984. The treatment of tinnitus--a historical perspective. J Laryngol Otol, 98, 963-72.

Wichmann, T. \& DeLong, M. R. 2016. Deep Brain Stimulation for Movement Disorders of Basal Ganglia Origin: Restoring Function or Functionality? Neurotherapeutics, 13, 264-83.

Wu, C., Martel, D. T. \& Shore, S. E. 2016. Increased Synchrony and Bursting of Dorsal Cochlear Nucleus Fusiform Cells Correlate with Tinnitus. J Neurosci, 36, 2068-73.

Zachriat, C. \& Kroner-Herwig, B. 2004. Treating chronic tinnitus: comparison of cognitive-behavioural and habituation-based treatments. Cogn Behav Ther, 33, 187-98.

Zoger, S., Svedlund, J. \& Holgers, K. M. 2006. Relationship between tinnitus severity and psychiatric disorders. Psychosomatics, 47, 282-8. 


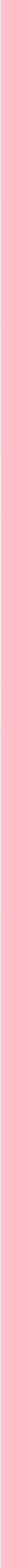




\section{Bhapter 2}

\section{Deep brain stimulation in tinnitus: current and future perspectives}

JV Smit, MLF Janssen, H Schulze, A Jahanshahi, JJ Van Overbeeke, Y Temel, RJ Stokroos Brain research, 1608 (2015) 51-65 


\section{Abstract}

Chronic tinnitus, also known as ringing in the ears, affects up to $15 \%$ of the adults and causes a serious socio-economic burden. At present, there is no treatment available which substantially reduces the perception of this phantom sound. In the past few years, preclinical and clinical studies have unraveled central mechanisms involved in the pathophysiology of tinnitus, replacing the classical periphery-based hypothesis. In subcortical auditory and non-auditory regions, increased spontaneous activity, neuronal bursting and synchrony were found. When reaching the auditory cortex, these neuronal alterations become perceptually relevant and consequently are perceived as phantom sound. A therapy with a potential to counteract deeply located pathological activity is deep brain stimulation, which has already been demonstrated to be effective in neurological diseases such as Parkinson's disease. In this review, several brain targets are discussed as possible tar gets for deep brain stimulation in tinnitus. The potential applicability of this treatment in tinnitus is discussed with examples from the preclinical field and clinical case studies. 


\section{Introduction}

Chronic subjective tinnitus currently affects up to $15 \%$ of the adult population (Heller, 2003, Møller, 2011), and its prevalence is increasing (Nondahl et al., 2012). The most severe degree of tinnitus is experienced in about $2.4 \%$ of the population (Axelsson and Ringdahl, 1989). Tinnitus is often associated with psychiatric disorders such as anxiety, insomnia and depression (Langguth et al., 2011, Zoger et al., 2006). In some cases, these behavioral symptoms lead to suicide (Coles, 1984, Lewis et al., 1994). Despite the severe impact tinnitus may have on the daily life of patients, no effective standard treatment is available to date that leads to a substantial remission of tinnitus.

It has been hypothesized that the substrate of tinnitus is located in the central nervous system (e.g. (Eggermont, 2003, Kaltenbach, 2000, Jastreboff, 1990, Rauschecker, 1999, Norena, 2011)) rather than in the periphery. Most of these hypotheses assume that initial damage to the peripheral receptor epithelium of the cochlea triggers maladaptive neuroplastic changes in the central auditory and limbic system that finally lead to the manifestation of chronic tinnitus. Based on these assumptions a number of neuromodulatory treatments, e.g. auditory cortex stimulation, direct electrical stimulation (De Ridder et al., 2007a) as well as transcranial magnetic stimulation (TMS) (Langguth et al., 2008) and stimulation of the auditory nerve (Holm et al., 2005) have been tested in search of an effective treatment against tinnitus. Some of these treatments have shown promising results. Yet, there is insufficient evidence that neuromodulation of the auditory nerve or auditory cortex causes long-term suppression of tinnitus (Langguth et al., 2008, Bartels et al., 2007). Stimulation with cochlear implants showed a substantial suppression of tinnitus in a group of patients (Arts et al., 2012). However, cochlear implants are currently solely applied in patients with bilateral severe sensorineural hearing loss, because of the risk of cochlear damage due to manipulation in the cochlea during surgery.

Recently, a more invasive neuromodulative technique, deep brain stimulation (DBS), has gained interest as a potential treatment for tinnitus (Cheung and Larson, 2010, Luo et al., 2012a, Shi et al., 2009). DBS involves the delivery of electrical currents to a selected area in the subcortical central nervous system. At present, DBS is applied in patients with refractory movement such as Parkinson's disease and essential tremor (Janssen et al., 2014a, Krack et al., 2003). DBS might be a potential treatment for refractory tinnitus if a selective neurophysiological and/or anatomical substrate could be identified (Soleymani et al., 2011, De Ridder and Møller, 2011, Hariz et al., 2013).

Nonetheless, several researchers already initiated animal and human studies to treat tinnitus with DBS in different targets (Cheung and Larson, 2010, Luo et al., 2012a). The most suitable brain structure for neuromodulatory treatment in tinnitus is still unclear. The goal of this review is to discuss possible targets for DBS therapy in tinnitus. To this aim we provide a comprehensive overview of studies that investigate tinnitus-related activity in auditory and non-auditory structures, but will first briefly outline the pathophysiology of tinnitus, animal models in tinnitus and the rationale behind neuromodulative therapy for tinnitus. 


\section{The origin of tinnitus percept}

Since it has been proven that eighth-nerve sectioning does not improve tinnitus (Barrs and Brackmann, 1984, House and Brackmann, 1981, Gardner, 1984), it is no longer thought that tinnitus is a purely peripheral phenomenon. Rather, it is presumed that there is a prominent involvement of structures in the central nervous system in the pathophysiology of tinnitus. This view is supported by a Positron Emission Tomography (PET)-study (Lockwood et al., 1998) where unilateral external tone bursts resulted in bilateral auditory cortex activation, whereas alteration of the loudness of unilateral tinnitus with oro-facial movements only showed contralateral cortical effects. If tinnitus had a cochlear origin, these patients should have shown bilateral activity during modulation of tinnitus.

The current hypothesis is that noise-induced tinnitus is triggered by injury of inner and/or outer ear hair cells. A tonotopically circumscribed decrease in auditory nerve activity leads to increased activity in the central nervous system accompanied by an increase in spontaneous firing rate, bursting activity, neural synchrony and formation of tonotopic reorganization (Eggermont, 2003, Kaltenbach, 2011, Norena and Eggermont, 2006, Chen and Jastreboff, 1995, Norena and Eggermont, 2003, Stolzberg et al., 2011). The mechanisms could be subsumed in the following hypothesis: the tonotopic organization of auditory structures will be reorganized due to deafferentation. In a normal tonotopic organization, groups of neurons mainly activate their associated ascending neurons with regard to their spectral tuning. In case of tonotopic reorganization, large numbers of neurons that formerly where predominantly activated by the deafferented neurons now receive input from the edge-frequency region. The neurons of this broader afferent network will now fire synchronously due to the common input and will show amplified baseline spontaneous activity. This altered activity may then elicit perception of tinnitus in the auditory cortex. The mechanisms that result in increased spontaneous activity likely involve either upregulation of excitatory input (Zeng et al., 2009) or a disinhibition due to decreased inhibition (Wang et al., 2009, Middleton et al., 2011). Thus, there is some central compensation for reduced auditory nerve activity, but this overall compensation comes at the price of a local imbalance between excitatory and inhibitory input to auditory structures that may lead to bursting activity, neural synchrony and tonotopic reorganization (Eggermont, 2006, Kaltenbach, 2011). These three mechanisms have been demonstrated in the primary auditory cortex (Komiya and Eggermont, 2000, Seki and Eggermont, 2003, Engineer et al., 2011) and inferior colliculus (IC) (Chen and Jastreboff, 1995, Bauer et al., 2008, Wang et al., 2002, Robertson et al., 2013). Moreover, increased bursting activity is found in the dorsal cochlear nucleus (DCN) (Brozoski et al., 2002, Kaltenbach et al., 2004), ventral cochlear nucleus (VCN) (Vogler et al., 2011) and medial geniculate body (MGB) (Basta et al., 2008). In addition, tonotopic reorganization is found in the MGB (Kamke et al., 2003). All these animal studies used methods that are able to induce tinnitus, but not all studies behaviorally tested for the actual presence of tinnitus. Some studies suggest that tonotopic reorganization may be a consequence of hearing loss and is not causally related to tinnitus (Langers et al., 2012, Yang et al., 2011). 
Brain networks consist of subnetworks which are intermingled, but each network could represent a specific aspect of tinnitus. The core tinnitus subnetwork involves the auditory pathways. Different characteristics of tinnitus like mood, distress and type probably involve several non-auditory, limbic structures (De Ridder et al., 2013). The limbic system has gained more interest in explanatory models for tinnitus; current models tend not only to explain auditory but also attentional and emotional components of tinnitus (Mahlke and Wallhäusser-Franke, 2004, De Ridder et al., 2006, Kraus and Canlon, 2012). Numerous models that link the altered neuronal network connectivity in tinnitus with the perception of tinnitus have been proposed. Nonetheless, the exact mechanism of maladaptive neural plasticity that leads to tinnitus remains unknown.

\section{Animal models for tinnitus and deep brain stimulation}

Human tinnitus studies are usually restricted to imaging studies, because histological and electrophysiological studies are difficult to perform in humans with regard to practical and ethical issues. Therefore, the majority of investigations in the field of tinnitus have been performed on animal models to unravel the mechanism behind tinnitus and search for new therapies. Animal models of tinnitus involve either salicylate administration or exposure to noise trauma, which both have been shown to induce tinnitus (Mongan et al., 1973) and are both accompanied by hearing loss (Day et al., 1989). The extent of hearing loss and tinnitus induced by these factors exerts a high inter-individual variation in both animals and humans. It should also be noticed that salicylates induce an acute and reversible tinnitus, while noise trauma induces chronic tinnitus (Eggermont, 2013).

Many experimental methods to test whether or not an animal perceives tinnitus involve basic mechanisms of operant conditioning (Lobarinas et al., 2004, Jastreboff and Sasaki, 1994, Bauer and Brozoski, 2001). The gap-detection prepulse inhibition (PPI) of the acoustic startle response (ASR) was introduced to provide evidence for the existence of a tinnitus percept (Turner et al., 2006), an approach that does not require pre-test training of the animal. This approach has the advantage that the maladaptive neuroplastic changes in the central nervous system that underlie the development of tinnitus may be investigated in the naïve brain and are not confounded by potential previous neuroplastic changes that are induced by conditioning paradigms.

Most of the animal studies investigated acute and not chronic tinnitus and the methods are usually restricted to the perceptual aspect of tinnitus. Moreover, it cannot be ruled out whether the perceptual measurements in animal models for tinnitus are confounded by hearing loss, temporal auditory processing deficits and/or hyperacusis. To minimize these confounders, the auditory brainstem response has been used to assess hearing thresholds. A minimization of a confounding effect of hearing loss in the startle paradigm can be achieved by dividing the gap condition with the normalized no-gap condition (Tziridis et al., 2014, Tziridis et al., 2012). However, the usefulness of the startle paradigm for 
tinnitus evaluation is still under debate (Eggermont, 2013, Lobarinas et al., 2012).

For DBS, the use of animal models is well-established. With coordinates from an animal brain atlas (Paxinos and Watson, 2007), custom-made electrodes can be precisely implanted in specific brain structures. Several brain areas have been stimulated to successfully treat various disorders (Tan et al., 2010).

\section{Neuromodulation in tinnitus}

\section{Deep brain stimulation in tinnitus-related structures}

DBS has been used clinically to treat several neurological diseases, such as Parkinson's disease and essential tremor. In an experimental setting, DBS has also been used to treat epilepsy, obsessive-compulsive disorder, Tourette's syndrome and pain. Similarities between tinnitus and pain in humans are frequently noted (Møller, 2007); especially for phantom pain. Neural plasticity is thought to play a key role in the development of both medical conditions. In this section, we will elaborate on preclinical and clinical studies that have investigated the effect of DBS on tinnitus. Later on, we will discuss side-effects that have occurred during DBS of potential tinnitus target structures, which were stimulated for other indications than tinnitus.

As stated above, the most common indication for DBS is Parkinson's disease. DBS of the subthalamic nucleus (STN) improves motor symptoms in both the short and long term for these patients (Krack et al., 2003, Deuschl et al., 2006, Janssen et al., 2014b). The clinical success of STN DBS in patients with Parkinson's disease can be attributed to the extensive preclinical and clinical research conducted before it was applied in humans (Temel et al., 2005, Benazzouz et al., 1993). Frequently, other diseases that have the potential to be treated with DBS, such as Huntington's disease (Temel et al., 2006), depression (Falowski et al., 2011) and also tinnitus (Luo et al., 2012), are explored in animals studies before clinical application. An animal study which assessed the effect of DBS on tinnitus used the gap-detection PPI of the ASR to assess tinnitus after exposing rats to unilateral noise trauma (Luo et al., 2012b). This study reported a suppression of tinnitus during DBS of the DCN but this finding, though promising, has to be interpreted with caution, since the sample size was small $(n=6)$, no sham group was included and the possible effects of hyperacusis or hearing loss were not taken into account. Furthermore, the effect of DBS on the ASR in healthy rats was not assessed. It can therefore not be ruled out that the results found are due to direct DBS effects on the PPI of the ASR rather than an alleviation of the tinnitus condition. Regardless of these limitations, this study stresses the importance of the DCN in the pathophysiology of tinnitus and urges further research for more conclusive results.

Two human studies have been conducted to assess the effect of DBS on tinnitus. One of these focused on the possible role of the ventral intermediate nucleus of the thalamus (VIM) in tinnitus. Seven patients received DBS implants in the VIM of the thalamus for movement disorders who also reported 
concomitant tinnitus (Shi et al., 2009). Three of seven patients experienced a decrease in tinnitus in DBS on versus DBS off condition. Tinnitus remained attenuated for at least 15 minutes after DBS was turned off. The patients reported no change in hearing. Alternatively to the hypothesis that stimulation of the VIM attenuates tinnitus, the current could have spread to the auditory nucleus of the thalamus, the MGB. A placebo effect could also not be ruled out, since the study was not blinded.

In the second human study that investigated the effect from DBS on tinnitus, the DBS lead was intra-operatively activated to stimulate a locus in the caudate nucleus ('area $L C$ '), before the target region, the STN or VIM, was reached to treat the motor symptoms of Parkinson's disease or essential tremor (Cheung and Larson, 2010). During DBS surgery, tinnitus was evaluated in six patients with long-standing tinnitus with sound quality description and loudness rating. Patients were blinded to amplitude changes in electrical stimulation. In five patients the DBS lead traversed or was adjacent to area LC (three bilateral) and one was placed just outside the caudate. Acute suppression of tinnitus during stimulation of the caudate was seen in all the patients except one patient in which the lead was outside the caudate nucleus. In bilateral implanted subjects, both sides were also subsequently stimulated but due to stimulation and/or microlesion carryover effect, it could not be determined which caudate had to be stimulated to suppress tinnitus. From the reported acute effects on tinnitus, it cannot be concluded whether the effect is really mediated by the area LC stimulation or by nearby structures which could be reached by stimulation of nearby passing fibers. Therefore, the results cannot be transferred to chronic treatment of patients with tinnitus as their primary symptom. Nevertheless, these results support the validity to initiate preclinical and subsequently clinical trials with tinnitus as primary indication for DBS.

\section{Mechanism of deep brain stimulation}

In DBS, a pulse generator which is placed subcutaneously under the clavicle or in the abdomen continuously sends electrical pulses to the DBS electrodes in the brain. The stimulation parameters of the pulse generator are controllable with a wireless device. The parameters are pulse width, stimulation amplitude and the frequency of the stimulation pulses and are optimized for each individual patient. Stimulation can be either mono- or bipolar.

The exact mechanism by which DBS exerts the therapeutic effect is not yet fully understood. However, several neural, cellular and sub-cellular mechanisms have been suggested, mainly based on DBS in Parkinson's disease (Montgomery and Gale, 2008). One possible mechanism is the silencing of neurons. This hypothesis is based upon the notion that high frequency stimulation of the VIM or STN has a similar functional effect as a lesion of the area (Hamani and Temel, 2012, Benabid et al., 1987, Benabid et al., 2002). In this respect, mechanisms such as depolarization block, jamming of the neural activity, channel blocking, neuronal energy deletion and synaptic failure have been suggested (Lozano et al., 2002). DBS is unlikely to provide only an inhibitory effect; probably DBS produces a combination of excitatory and inhibitory effects (Vitek, 2002, McIntyre et al., 2004). The neurons surrounding the electrode 
may be inhibited, while the output axons of local neurons are stimulated (Grill et al., 2004). At resting conditions, the nerve membrane potential of $-70 \mathrm{mV}$ results from the difference between the interior of a cell with a negative baseline voltage relative to a positive exterior baseline voltage. A cathode, such as the electrodes in DBS, can pull the positive charges away from the outside of the membrane, thereby inducing an action potential (Benazzouz and Hallett, 2000). The injected electrical activity may result in modifications of pathological spontaneous activity in various nuclei (Hammond et al., 2008, Montgomery and Gale, 2008, Meissner et al., 2005). In line with these hypotheses, a number of studies have shown that DBS induces release of neurotransmitters from axon terminals. Animal studies have suggested a release of GABA (Boraud et al., 1996, Windels et al., 2005) and glutamate (Boulet et al., 2006, Windels et al., 2003). Human studies have suggested the release of GABA (Dostrovsky et al., 2000) and cyclic GMP, but not that of glutamate (Stefani et al., 2005).The most recent theory of the mechanism behind DBS, at least in STN stimulation for Parkinson's disease, is that electrical stimulation may act by modulating pathological patterns of synchronized oscillations (Kuhn et al., 2008, McIntyre and Hahn, 2010).

The complex mechanism of DBS entails that each individual brain structure may have its own response to DBS which could result in different outcomes (Benazzouz and Hallett, 2000). In tinnitus spontaneous activity and neuronal synchrony is increased in several auditory and non-auditory structures. Obviously tinnitus is not a condition which can be related to one structure within or related to the auditory pathways, but is a result of the interaction between different nuclei within auditory and non-auditory circuits. DBS at one of those target areas influences pathological neuronal activity which may result in the attenuation of tinnitus by suppression of pathologically increased, tinnitus-related neuronal activity or disruption of the oscillatory activity.

Tinnitus-related hyperactivity in the $\mathrm{CN}$ and IC is likely mediated by a decrease in GABAergic inhibition (Middleton et al., 2011, Dong et al., 2009, Suneja et al., 1998). The suggested release from GABA following DBS might supplement the GABA deficiency in tinnitus. Interestingly, impairment in GABAergic neurotransmission is also found in Parkinson's disease (Mallet et al., 2006) from which it is known that symptoms are improved during DBS.

In rats with induced acoustic trauma, spontaneous bursting activity is increased in the VCN and MGB (Chang et al., 2002, Finlayson and Kaltenbach, 2009, Vogler et al., 2011, Basta et al., 2008). In two studies that tested for tinnitus after acoustic trauma, the DCN also showed an increased spontaneous bursting activity (Brozoski et al., 2002, Kaltenbach et al., 2004). Such an increase in bursting activity might be a good predictor of a promising target for DBS, because the increased bursting activity found in the STN is pathognomonic in Parkinson's disease patients and animals and is reduced by DBS (Bergman et al., 1994, Janssen et al., 2012, Benazzouz et al., 2002).

High frequency stimulation is probably the right modality of stimulation for tinnitus, as this has been shown to be effective in hyperactive structures, as seen in the STN in Parkinson's disease (Benazzouz et 
al., 1995). Whether this applies for all auditory and non-auditory structures still has to be demonstrated. For example, one of the differences between the two parallel auditory pathways is the classical being tonotopic and the non-classical being less tonotopic. This may have clinical implications, indicated by the hypothesis that white-noise tinnitus may be caused by synchronous hyperactivity of burst firing in the non-tonotopic non-classical pathway whereas pure-tone tinnitus may result from increased synchronous tonic firing in the tonotopic classical pathway (De Ridder et al., 2007c). This suggests that the classical and nonclassical pathway may require different stimulation paradigms. Since tinnitus is probably caused by multiple changes in the whole auditory network and not specifically related to one of the nuclei, we believe that DBS of several structures might influence tinnitus; stimulation in any of these structures might disrupt the pathological mechanisms and interactions that manifest within the network as a whole.

\section{Noninvasive neuromodulation in tinnitus}

A possible alternative to DBS that follows similar mechanistic approaches to alleviate tinnitus are attempts to noninvasively manipulate brain activity. In this context, two main methods have been tested so far.

Repetitive transcranial magnetic stimulation (rTMS) is a noninvasive method to stimulate superficial cortical areas. At this moment rTMS in the auditory cortex is performed in clinical studies to treat tinnitus. So far, the effects of rTMS remain controversial. Randomized studies show either a small improvement in tinnitus scales (Rossi et al., 2007, Plewnia et al., 2007a, Kleinjung et al., 2005, Kim et al., 2014) or no effect at all (Piccirillo et al., 2011, Plewnia et al., 2007b, Smith et al., 2007, Hoekstra et al., 2013). rTMS can also elicit tinnitus (Benninger et al., 2011), which was also shown by DBS (Larson and Cheung, 2012).

Another non-invasive form of neuromodulation is transcranial direct current stimulation (tDCS). A recent meta-analysis, in which two randomized clinical trials and three open-label studies were analyzed, showed that $39.5 \%$ of the tinnitus patients responded to tDCS to the prefrontal and temporal areas with a mean tinnitus intensity reduction of 13.5\% (Song et al., 2012). The tinnitus intensity, however, does not always correlate with the impact of tinnitus in daily life (Baskill and Coles, 1999). The only study that evaluated the effect of tDCS on the impact of life is an open-label study (Frank et al., 2012) which showed no decrease after tDCS. At the moment, the efficacy of tDCS for treating tinnitus remains unclear.

The major advantage of these techniques is the non-invasive character. Therefore further research in the efficacy is worthwhile and should be encouraged in parallel to DBS research. 


\section{Potential DBS targets in the auditory pathways}

The brain regions involved in tinnitus can be assigned to the auditory or non-auditory pathways (Figure 1). Here, we will focus on various brain structures within the auditory pathways and their relation to tinnitus. For every structure, animal studies will be discussed first, followed by human studies. Based on these findings, we will discuss whether or not stimulation of these structures has the potential to be explored as a treatment target for tinnitus and debate potential side-effects.

Despite the fact that the auditory cortex has an important role in the perception of tinnitus, we will focus on subcortical structures. For neuromodulation for tinnitus in cortical areas, we refer to other literature (De Ridder et al., 2007a, De Ridder and Vanneste, 2011).

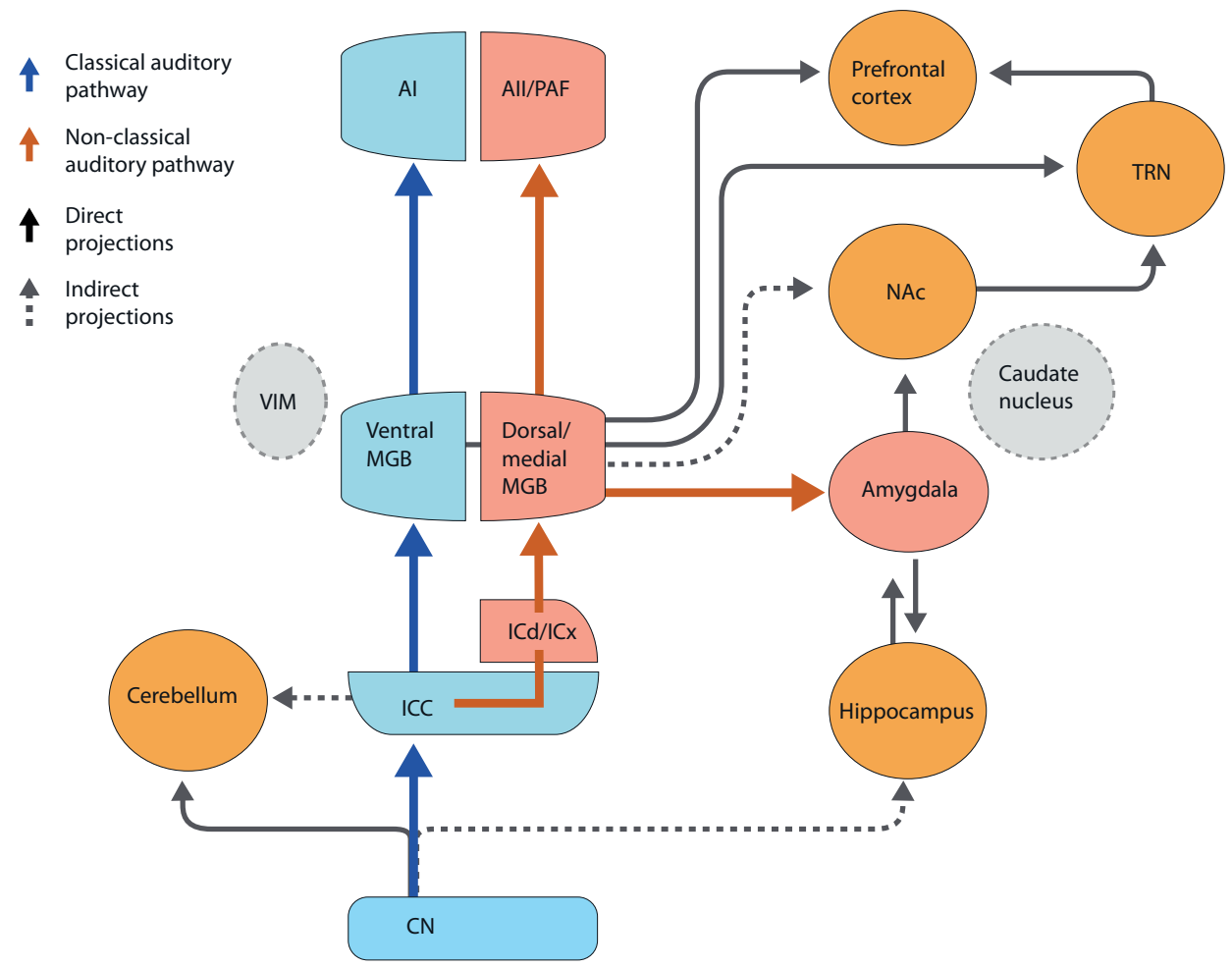

Figure 1. Tinnitus related auditory and non-auditory structures with ascending projections. The auditory pathways can further be subdivided in a classical and a non-classical pathway. In the classical pathway the axons from the cochlear nerve terminate in the ipsilateral CN. The CN projects to the ICC and the cells of the ICC project to the ventral part of the MGB in the auditory thalamic nucleus. The MGB is the auditory sensory nucleus of the thalamus and a relay station between the $I C$ and the auditory cortex. The $M G B$ is located in the posterior surface of the thalamus and has three divisions: ventral, medial and dorsal. Whilst the ventral division is specific to the classical auditory pathway, the dorsal and medial divisions are part of the non-classical pathway. In the non-classical 
pathway, the central nucleus of the IC is connected to the external and dorsal nuclei of the IC (ICX and ICd, respectively). The cells in the ICX and ICd project to medial and dorsal parts of the MGB. The nontonotopic dorsal and medial divisions of the MGB projects to the amygdala and caudate nucleus (Kandel et al., 2000; Møller, 2011b). Whereas the classical pathway projects to the primary auditory cortex, the non-classical pathway projects directly to the secondary and association auditory cortices. Additionally, the cells project from the medial and dorsal parts of the thalamus directly to limbic structures like the amygdala. In the classical pathway, the amygdala receives afferent input from the auditory association cortex that receives input from the primary auditory cortex (LeDoux, 1992a; Møller, 2011b). Note that for clarity descending projections and projections from non-auditory structures to auditory pathways are not denoted. Al = primary auditory cortex, $A I I=$ secondary auditory cortex, CN = cochlear nucleus, ICC, ICd and ICX are respectively the central, dorsal and external nucleus of the inferior colliculus, MGB = medial geniculate body of the thalamus, NAc = nucleus accumbens, PAF = posterior auditory cortical field, TRN = thalamic reticular nucleus, VIM = ventral intermediate nucleus of the thalamus.

\section{Cochlear nucleus}

The DCN fulfills a prominent role in sound localization (May, 2000), improvement of auditory processing in the presence of background noise (Frisina et al., 1994) and is probably involved in echo suppression (Wickesberg and Oertel, 1990). Increased spontaneous activity in the DCN induced by noise was found in several animal studies, whereby some studies also measured the presence of tinnitus. This spontaneous neural activity becomes chronically elevated (reviewed by (Kaltenbach, 2006) and (Tzounopoulos, 2008)). The DCN is often reported as an important structure in the generation of tinnitus (Levine, 1999, Kaltenbach et al., 2005). The origin of the increased activity in the DCN might be a plasticity-related process, as demonstrated by increased expression of doublecortin, a marker of neuronal precursor cells that is linked to plasticity (Manohar et al., 2012, Bauer et al., 2013). If the DCN is a generator of tinnitus, ablation of this structure would consequently decrease activity of higher output structures. This is indeed the case: ablation of the DCN resulted in a major reduction of noise trauma related hyperactivity of the IC (Manzoor et al., 2012). A recent study assessed the presence of tinnitus in rats, and instead of ablating the DCN, they electrically stimulated the structure (Luo et al., 2012a). This animal study showed in a small group of animals a suppression of tinnitus using the gap-detection PPI of the ASR. As previously mentioned, there were several limitations and therefore the results need to be validated. Contrary to the hypothesis that the DCN serves as a tinnitus generator, ablation of the DCN in rats did not decrease tinnitus, measured with the operant conditioning model (Brozoski and Bauer, 2005). Although the whole area could not be ablated, the study suggested that the DCN serves as a tinnitus trigger, rather than a generator. To test if the DCN acts as a trigger for tinnitus, the DCN in rats were ablated bilaterally prior to tinnitus induction (Brozoski et al., 2011). In this case the ablation prevented the development of tinnitus. These results imply that it is likely that the DCN indeed serves as a trigger, but is not the right target to treat tinnitus.

Less is known about the role of the VCN in tinnitus. Increased spontaneous firing rate in the VCN after inducing cochlear trauma has only been shown in a guinea pig model which did not measure tinnitus (Vogler et al., 2011). Magnetic resonance imaging (MRI) with administration of the contrast agent manganese, which accumulates in active neurons, showed the VCN as an active area in rats with behavioral signs of tinnitus (Brozoski et al., 2007). 
Human studies that described the role of the $\mathrm{CN}$ in tinnitus are scarce. Human imaging studies have not yet succeeded in showing increased spontaneous activity due to the poor spatial resolution compared to the small size of the structures and the poor signal-to-noise ratio in brainstem imaging (Lanting et al., 2009, Hawley et al., 2005). Only one imaging study showed an increase in PET activity in the CN when a patient increased his tinnitus perception by lateral gaze (Lockwood et al., 2001). Nevertheless, the first indication that the DCN plays an important role in tinnitus was assessed in a human study (Soussi and Otto, 1994). Eighteen patients underwent implantation of an auditory brainstem implant in the DCN after bilateral acoustic neurofibroma surgery during which the auditory nerve often loses function. The implant consisted of two electrodes placed over the cochlear nucleus complex. Six out of a total of ten patients who used the implant successfully reported reduction during stimulation and one patient reported complete suppression of tinnitus. However, it has to be considered that the effects in these patients who suffered from complete auditory deafferentation may not be transferred to other tinnitus patients who still receive auditory input.

Side-effects while stimulating the $\mathrm{CN}$ are investigated in a study where an auditory brainstem implant was inserted into the $\mathrm{CN}$ in cases where the auditory nerve lost function (Zhang and Zhang, 2010). Side effects after auditory brainstem implantation are seen in some cases (Soussi and Otto, 1994) while other studies report no side effects (Matthies et al., 2000). Reported side effects include facial pain and ocular vibration. Because hearing loss was already present in these patients, it remains to be investigated whether or not stimulation of the $\mathrm{CN}$ impairs hearing.

In conclusion. the DCN is the first and thus far only target that has successfully been stimulated with DBS to attenuate tinnitus in an animal model (Luo et al., 2012a). Although from a surgical point of view it is a challenging area for stimulation, it has the potential to be targeted with DBS. The VCN is less frequently investigated in relation to tinnitus and more animal studies are necessary to reveal the role of the VCN in the neural basis of tinnitus.

\section{Inferior colliculus}

The cochlear nucleus projects to the central nucleus of the IC (ICC). These connections are mainly crossed; however there are also ipsilateral projections to the ICC. The ICC is a converging center for almost all ascending auditory brainstem projections, thereby integrating all these different streams of auditory information (Kandel et al., 2000). Several animal studies were performed in which local field potentials were measured with implanted electrodes in the IC. Increased contralateral neuronal spontaneous activity and neural synchrony have been demonstrated in the ICC in chinchillas with behavioral evidence of tinnitus (Bauer et al., 2008). The external nucleus of the IC (ICX), which is part of the non-classical auditory pathway, showed an increase in spontaneous firing rate, specifically bursting activity, in rats with salicylate-induced tinnitus (Chen and Jastreboff, 1995). The activity could be embedded in a reorganization of the tonotopic map (Salvi et al., 1996, Harrison, 2001, Wang et al., 2002, Imig and Durham, 2005). Functional MRI (fMRI) studies confirm the previously mentioned hyperactivity 
in rats with tinnitus, showing elevated activity in the IC (Brozoski et al., 2007). In addition, PET imaging in rats with tinnitus showed increased activity in the IC (Paul et al., 2009). Evidence of enhanced activity in the IC in humans is particularly based on FMRI studies where sound-evoked activity changes can be measured. In these studies, the IC in patients with tinnitus showed altered sound-evoked activity compared with control groups (van Gendt et al., 2012, Lanting et al., 2008, Melcher et al., 2000, Smits et al., 2007). A structural MRI study revealed a grey matter decrease in the IC in tinnitus patients with normal hearing (Landgrebe et al., 2009).

Side-effects of IC stimulation have been studied with the auditory midbrain implant. The auditory midbrain implant has been investigated for the purpose of improving the poor hearing outcome that is often seen in patients with auditory brainstem implants that stimulate the CN. Until today, only three patients underwent such surgery (Lim et al., 2008). All patients underwent unilateral acoustic neuroma removal and where already deaf at this side before surgery. Side-effects included the perception of unpleasant sounds, paresthesia, temperature change, dizziness and facial twitches. However, these side-effects all diminished by turning off corresponding electrodes. The remaining active electrodes elicited perception of sounds, some with broad spectral properties. Interestingly, only perception of low frequency sounds could be evoked.

One of the models for tinnitus development involving the IC is the auditory brain stem model (Gerken, 1996). In this model lateral inhibition is assumed to exaggerate unevenness in spontaneous firing rate across frequencies, so that reduced spontaneous firing rate as seen in hearing loss may lead to disinhibition in frequency channels adjacent to the damaged site. This hyper-responsiveness in the IC might be the basis for tinnitus. On the other hand, because the IC is not the first station in the auditory pathway that shows increased activity, it may be hypothesized that the IC is just a passive relay station whereas the generation of activity originates from the $\mathrm{CN}$. This latter hypothesis is confirmed by a noise-induced animal model where electrophysiological measurements showed similar activity profiles in the IC and CN (Manzoor et al., 2012, Imig and Durham, 2005). Alternatively, it may also be assumed that the described mechanism on lateral inhibition works on every level of the auditory pathway, enhancing the disinhibition step by step.

In summary, no human or animal studies on IC DBS for tinnitus are available. However, the prominent changes seen in the IC in animal models of tinnitus, such as an increase in spontaneous firing rate and bursting activity, make the IC a promising target for DBS.

\section{Medial geniculate body}

Only a few animal studies link the MGB to tinnitus. An in vitro study from rats with salicylate-induced tinnitus showed that the intrinsic properties of the dorsal and ventral MGB neurons were changed and the evoked response by stimulation of the IC was reduced (Su et al., 2012). Several animal studies concerning the MGB did not assess the presence of tinnitus, which makes it impossible to solely 
interpret these results as influenced by tinnitus. Hyperacusis and hearing loss could have influenced the results. In these studies, neuronal hyperactivity has been found, but to a lesser extent than in other regions. After noise-exposure in mice, the neuronal cell density reduced in all divisions of the MGB (Basta et al., 2005). Similar as in the IC, a tonotopic reorganization has been found in the MGB in animals with cochlear lesions (Kamke et al., 2003).

Evidence for neuronal hyperpolarization in the MGB is found in an $\mathrm{FMRI}$ study with gaze-evoked tinnitus patients due to surgical extirpation of a vestibular schwannoma. The MGB showed a decrease in BOLD-response (van Gendt et al., 2012). This could, together with the reduced inhibition that was found in the auditory cortex, be explained by thalamocortical dysrhythmia. In other human imaging studies, the MGB also showed altered activity related to tinnitus. One fMRI study demonstrated significant signal change in the MGB in patients with tinnitus (Smits et al., 2007). In a PET-study, a small group of patients with right ear tinnitus showed increased neuronal metabolism in the right thalamus including the MGB, after modulating tinnitus with oro-facial movements (Lockwood et al., 1998). An increase in grey matter concentration in the posterior thalamus including the MGB was found in a structural MRI study (Mühlau et al., 2006).

Up to date, side-effects are unknown since there have been no clinical studies conducted involving electrical stimulation of the MGB. Thalamic stimulation of the VIM however has shown to be very effective for tremor with only low risks of stimulation induced side-effects (Koller et al., 2001).

The role of the MGB in tinnitus is not as intensively investigated as those of the $\mathrm{CN}$ and the IC. Nevertheless, integration of limbic and auditory information occurs at the level of the thalamus which suggests a role for the MGB, or other parts in the thalamus, in the multisensory integration in tinnitus. Furthermore, DBS in the MGB offers potential benefit in tinnitus patients because of its possible interference with the thalamocortical loop (Hammond et al., 2008). Finally, from a surgical point of view the MGB is a target which is relatively easy to access with relatively low surgical risks compared to structures in the brainstem.

\section{Potential DBS targets in non-auditory pathways}

Multiple areas in both the auditory and non-auditory pathways are likely to be involved in tinnitus. Despite the fact that several animal studies have contributed to the understanding of the pathophysiology of tinnitus, the exact mechanisms of this pathophysiology are still not fully understood. The pathophysiology of tinnitus is not limited to auditory structures within the (non-)classical pathway, but also involves other brain regions. A role for the non-auditory system in tinnitus is supported by the notion that tinnitus loudness does not always correlate with measurements of severity (Meikle et al., 1984). The limbic system, specifically the amygdala, might play a key role in the perception and chronic manifestation of tinnitus (Wallhäusser-Franke et al., 2003, De Ridder et al., 2006). In particular, 
the limbic system may be able to suppress plasticity-induced spontaneous activity that otherwise can cause tinnitus (Rauschecker et al., 2010). Interaction with the limbic system may explain associated symptoms of tinnitus such as sleep problems, anxiety, depression and fear (Jastreboff, 1990, Kraus and Canlon, 2012). The emotional attributes rather than the tinnitus itself are of great relevance for the patients' daily life. This also may explain why cognitive therapy can improve quality of life, but does not improve the subjective loudness of tinnitus (Martinez Devesa et al., 2007). The role in tinnitus of two other non-auditory structures, the VIM and the caudate nucleus, has been discovered in patients with Parkinson's disease treated with DBS. The hippocampus, another limbic structure, and the nucleus accumbens (NAc), closely related to the limbic system, might also be involved in tinnitus as well as the cerebellum, which has been suggested to be a possible generator of tinnitus.

\section{Limbic structures}

Human fMRI studies have demonstrated that both the amygdala and hippocampus are involved in tinnitus perception (Shulman et al., 1995). The role of the amygdala in tinnitus has been underpinned by several clinical and animal studies. When the ipsilateral amygdala and hippocampus where temporally inactivated by barbiturare injection, 10-30\% suppression of tinnitus was reported by three of six patients, contralateral inactivation resulted in 10-100\% suppression of tinnitus in four out of six patients (De Ridder et al., 2006). Also animal studies show evidence for the involvement of the amygdala in tinnitus. In gerbils arg3.1, a plasticity protein, and c-fos are both up-regulated in the amygdala after salicylate injections (Mahlke and Wallhäusser-Franke, 2004). Systemic salicylate administration in rats showed hyperactivity and tonotopic shifts in the amygdala. Additionally, salicylate administration directly into the amygdala enhanced sound-evoked activity in the auditory cortex (Chen et al., 2012).

Direct electrical stimulation of the human amygdala has been performed in patients with medically refractory epilepsy for presurgical evaluation and in some patients evoked negative emotions like fear, anxiety and sadness (Lanteaume et al., 2007).

The possible neuropsychiatric side effects of DBS these structures outweigh their involvement in tinnitus generation and are therefore not suitable targets for DBS in tinnitus patients.

\section{Caudate nucleus}

The body and tail of the caudate nucleus receives projections from the secondary auditory area and association cortex (Yeterian and Pandya, 1998, Selemon and Goldman-Rakic, 1985). To our knowledge, no animal studies have been performed that link the caudate nucleus to tinnitus so the motivation to consider the caudate as a potential DBS target is solely related to clinical case studies. A case report reported tinnitus suppression after a cerebrovascular accident including the putamen and caudate nucleus (Lowry et al., 2004). Another case report that suggests a possible role of this region in tinnitus describes a patient which had a perioperative focal vascular injury in area LC of the caudate, a locus 
of the caudate positioned anterior to the dorsal striatum, that resulted in a complete attenuation of tinnitus (Larson and Cheung, 2013). In light of these findings a study was performed in which the area LC was stimulated intra-operatively, before the final target region was reached (Cheung and Larson, 2010). As already previously discussed, tinnitus loudness decreased in five out of six patients. It should however be noted that in this study tinnitus was reported on a 0-10 scale rather than a validated tinnitus severity rating instrument like the THI (Cheung and Larson, 2010). In another study, three patients with tinnitus and three patients without tinnitus underwent DBS for movement disorders and the DBS lead was again temporarily targeted to area LC (Larson and Cheung, 2012). In all but one patient new sound sensations were triggered with various stimulation settings. Adverse effects after implantation of an electrode in the caudate nucleus were tracked in a case study (Aouizerate et al., 2004) where the caudate nucleus was successfully stimulated for obsessive compulsive disorder and no cognitive deterioration or adverse clinical effects were noted.

It is reasonable to assume that the caudate nucleus is one of potential areas to target with DBS. However, unambiguous evidence in support of this assumption is still lacking. Nonetheless, the results from the single clinical study that showed that DBS of the caudate nucleus suppressed tinnitus are encouraging.

\section{Nucleus accumbens}

The NAc is part of the ventral striatum and receives input from the thalamus, major limbic and cognitive areas of the brain (Basar et al., 2010, Kandel et al., 2000). A relation to the auditory system is thought to arise from axons from the NAc that innervate the thalamic reticular nucleus (O'Donnell et al., 1997). In a comparison study of $\mathrm{fMRI}$ experiments between tinnitus patients and controls, the NAc showed a bigger sound-evoked BOLD-response in tinnitus patients, specifically to sounds frequency-matched to patients' tinnitus (Leaver et al., 2011). The subcallosal area, including the NAc, has been shown to possess volume loss in tinnitus patients (Mühlau et al., 2006). The NAc also is considered important in tinnitus distress, as shown in an EEG-study (Vanneste et al., 2010). A model for tinnitus involving the limbic system has recently been hypothesized (Rauschecker et al., 2010). According to this hypothesis, the subcallosal area of the NAc becomes dysfunctional and tinnitus can no longer be inhibited. DBS of the NAc is proposed to counteract this dysfunctional area. In their view the NAc should be hyperactivated in order to induce a noise cancellation effect.

Direct stimulation of the NAc for treatment of obsessive-compulsive disorder often showed hypomania as a stimulation-related side-effect (de Koning et al., 2011). When the NAc is targeted for tinnitus, patients should be screened by a psychiatrist on forehand and be carefully monitored to recognize hypomanic symptoms.

In summary, there is some evidence for involvement of the NAc in tinnitus. The interesting model which involves the limbic system in tinnitus is encouraging to further investigate the role of the NAc in tinnitus. The effect and mechanism of electrical stimulation of the NAc should be tested in animal studies before 
clinical trials are conducted.

\section{Thalamus}

Thalamotomies have been performed for treatment of pain (Richardson, 1967). Intriguingly, thalamotomies not only reduced pain, but also attenuate tinnitus (Jeanmonod et al., 1996). As previously noted, tinnitus has some similarities with phantom pain. DBS is performed for pain relief in several studies. In patients with medial thalamotomies a $50-100 \%$ relief in tinnitus was obtained in three of six patients with solely tinnitus or tinnitus combined with neurogenic pain. One patient experienced complete relief from tinnitus (Jeanmonod et al., 1996). It cannot be ruled out that more structures in the thalamus than solely the MGB are involved in at least some forms of tinnitus; the VIM and the thalamic reticular nucleus (TRN) are both non-auditory thalamic structures that have been associated with tinnitus.

To our knowledge, no animal studies have been performed that link the VIM to tinnitus. As previously discussed, one human tinnitus study investigated the effect of VIM stimulation on tinnitus. When the DBS was turned on, three of seven patients experienced a decrease in tinnitus (Shi et al., 2009). Stimulating the VIM in Parkinson's disease and patients with essential tremor showed mild adverse effects like paraesthesia, limb dystonia, dysarthria, disequilibrium and subtle cognitive defects (Benabid et al., 1993). This study confirms the feasibility of DBS for tinnitus. However, because tinnitus was only suppressed in a subgroup of patients, it remains necessary to determine the differences between these patients and to find other more successful areas for neuromodulatory treatment.

The TRN has been linked to tinnitus as well. Although there is no direct evidence that the TRN shows altered activity in tinnitus, the structure is incorporated in the tinnitus model proposed by Rauscheker (Rauschecker et al., 2010) which is mostly based on imaging and anatomical studies.

The importance of the thalamus in tinnitus is suggested in multiple studies which involve non-auditory thalamic regions as well as auditory thalamic regions. With respect to neuromodulation, the clinical case series show some potential for targeting the thalamic nuclei for DBS treatment (Shi et al., 2009).

\section{Cerebellum}

The cerebellum is connected to several auditory structures (Petacchi et al., 2005, Aitkin and Boyd, 1975, Snider and Stowel, 1944, Huang et al., 1982). One of the areas in the cerebellum that is associated with the auditory system is the paraflocculus, which elicits evoked responses from auditory stimuli (Azizi et al., 1985) and showed elevated activity in a MRI study in rats (Brozoski et al., 2007). After the cerebellar paraflocculus ablation in rats, tinnitus was completely eliminated. Interestingly, when the cerebellar paraflocculus was ablated prior to tinnitus induction, tinnitus was not reduced. The rats still developed tinnitus, but to a lesser extent. A drawback of this target is occurrence of vestibular side-effects 
which were seen in some rats (Bauer et al., 2012). Local injection of glutamatergic antagonists in the paraflocculus resulted in reduced tinnitus (Bauer et al., 2013).

In humans, single photon emission computerized tomography (SPECT) and PET studies in patients with tinnitus showed significantly increased blood flow in the cerebellum (Osaki et al., 2005, Shulman and Strashun, 1999). An fMRI study showed increased blood flow in the cerebellum during tinnitus (Boyen et al., 2014). In addition to imaging studies, the cerebellum is also one of the areas that, like the auditory cortex, showed significant oscillatory power changes in magnetoencephalography (Sedley et al., 2012).

Chronic electrical stimulation of the cerebellum has been performed in patients with medically refractory epilepsy with limited side-effects (Fountas et al., 2010). Though, the paraflocculus, which recently has been suggested as an important structure in tinnitus (Bauer et al., 2012) has never been targeted for electrical stimulation in humans. Therefore no information is available on possible side-effects.

The cerebellum was for a long time not much discussed in relation to tinnitus. However, recently conducted animal research showed that the cerebellum might contribute to the magnitude of tinnitus (Bauer et al., 2012). Nonetheless, more information on the relation of the cerebellum with tinnitus is needed before this structure can be considered as a potential DBS target for tinnitus.

\section{Ethical considerations}

Despite the fact that there is currently no treatment available that fully attenuates tinnitus, there are some treatment strategies that help the patients to cope with tinnitus. Those treatments are based on cognitive behavioral therapy (Cima et al., 2012) and/or education in combination to exposure to an external sound (Jastreboff, 1999, Okamoto et al., 2010). A treatment that could be helpful in treating tinnitus in patients with profound to severe sensorineural hearing loss is the cochlear implant, which in a number of patients can attenuate tinnitus in addition to providing a sense of sound (Arts et al., 2012). The future place for DBS in the treatment of tinnitus should not replace such therapies but could serve as a last resort for therapy-resistant patients who are severely suffering from their tinnitus. DBS is a minimal-invasive and reversible type of functional neurosurgery. Nonetheless, complications of the surgery, hardware failure and of stimulation-related side effects should be bared in mind. The potential risk of complications should be carefully weighed against the potential benefits of DBS.

Regardless of the risks that are associated with the surgery, worldwide over 100.000 patients are currently being treated with DBS, mainly for movement disorders. The willingness of patients to be treated with DBS is essential. Surgeries for the main goal of attenuation of tinnitus have been performed, like cortical implantation (De Ridder et al., 2008), cochlear implants (Van de Heyning et al., 2008), and several types of surgeries for pulsatile tinnitus (De Ridder et al., 2007b). More specific to 
DBS, a questionnaire study was performed which assessed whether patients were willing to undergo DBS if they knew it would completely eliminates their tinnitus (Tyler, 2012). Out of 197 patients, around 40 would definitely accept this treatment. If their tinnitus loudness and annoyance would be reduced by half, $12.7 \%$ of the patients are very interested in DBS.

\section{Conclusion}

DBS is a potential treatment for tinnitus and has already been shown to be able to suppress tinnitus in a few studies. Human studies which investigate chronic DBS for the primary indication of tinnitus have not yet been performed. However, DBS has been suggested to be effective in both auditory and non-auditory brain structures. One animal study showed that DBS of the CN attenuated tinnitus related behavior. Based on studies that found pathological neuronal activity in subcortical auditory structures, similar effects of DBS in the IC and MGB may be expected. The CN and IC are surgically challenging to reach with electrodes but chronic stimulation has already been performed for hearing aid implantations. The MGB is easily targeted using the classical stereotactical approach. DBS is hereby proposed to interfere with the pathological neuronal activity by an overall suppressive inhibitory effect and/or interfere in the pathological oscillatory tinnitus network loop. Non-auditory structures with probably the most potential are the caudate nucleus and the VIM, which already have been studied in patients with Parkinson's disease. Other non-auditory structures such as the nucleus accumbens are involved in tinnitus and could be feasible potential targets, but more research is necessary to explore their precise role in the pathophysiology of tinnitus, before studies on DBS are valuable.

In conclusion, a subgroup of tinnitus patients seems to be eligible for DBS treatment. For this group, the potential risk of complications should be carefully weighed against the potential benefits of DBS. Side-effects of DBS, in the case of auditory and non-auditory target structures, have to be thoroughly evaluated before DBS may be applied to the clinic. To overcome these barriers, preclinical and clinical studies are needed to determine the optimal DBS target and stimulation parameters. 


\section{References}

Aitkin, L. M. \& Boyd, J. 1975. Responses of single units in cerebellar vermis of the cat to monaural and binaural stimuli. J Neurophysiol, $38,418-29$.

Aouizerate, B., Cuny, E., Martin-Guehl, C., Guehl, D., Amieva, H., Benazzouz, A., Fabrigoule, C., Allard, M., Rougier, A., Bioulac, B., Tignol, J. \& Burbaud, P. 2004. Deep brain stimulation of the ventral caudate nucleus in the treatment of obsessive-compulsive disorder and major depression. Case report. J Neurosurg, 101, 682-6.

Arts, R. A., George, E. L., Stokroos, R. J. \& Vermeire, K. 2012. Review: cochlear implants as a treatment of tinnitus in single-sided deafness. Curr Opin Otolaryngol Head Neck Surg, 20, 398-403.

Axelsson, A. \& Ringdahl, A. 1989. Tinnitus--a study of its prevalence and characteristics. Br J Audiol, 23, 53-62.

Azizi, S. A., Burne, R. A. \& Woodward, D. J. 1985. The auditory corticopontocerebellar projection in the rat: inputs to the paraflocculus and midvermis. An anatomical and physiological study. Exp Brain Res, 59, 36-49.

Barrs, D. M. \& Brackmann, D. E. 1984. Translabyrinthine nerve section: effect on tinnitus. J. Laryngol. Otol., Suppl. 9, $287-293$.

Bartels, H., Staal, M. J., Holm, A. F., Mooij, J. J. \& Albers, F. W. 2007. Long-term evaluation of treatment of chronic, therapeutically refractory tinnitus by neurostimulation. Stereotact Funct Neurosurg, 85, 150-7.

Basar, K., Sesia, T., Groenewegen, H., Steinbusch, H. W., Visser-Vandewalle, V. \& Temel, Y. 2010. Nucleus accumbens and impulsivity. Prog Neurobiol, 92, 533-57.

Baskill, J. L. \& Coles, R. R. A. 1999. Relationships between tinnitus loudness and severity. Sixth International Tinnitus Seminar, Cambridge, UK, Tinnitus and Hyperacusis Centre, London, 424-428.

Basta, D., Goetze, R. \& Ernst, A. 2008. Effects of salicylate application on the spontaneous activity in brain slices of the mouse cochlear nucleus, medial geniculate body and primary auditory cortex. Hear Res, 240, 42-51.

Basta, D., Tzschentke, B. \& Ernst, A. 2005. Noise-induced cell death in the mouse medial geniculate body and primary auditory cortex. Neurosci Lett, 381, 199-204.

Bauer, C. A. \& Brozoski, T. J. 2001. Assessing tinnitus and prospective tinnitus therapeutics using a psychophysical animal model. J Assoc Res Otolaryngol, 2, 54-64.

Bauer, C. A., Kurt, W., Sybert, L. T. \& Brozoski, T. J. 2012. The cerebellum as a novel tinnitus generator. Hearing Research.

Bauer, C. A., Turner, J. G., Caspary, D. M., Myers, K. S. \& Brozoski, T. J. 2008. Tinnitus and inferior colliculus activity in chinchillas related to three distinct patterns of cochlear trauma. J Neurosci Res, 86, 2564-78.

Bauer, C. A., Wisner, K. W., Baizer, J. S. \& Brozoski, T. J. 2013. Tinnitus, unipolar brush cells, and cerebellar glutamatergic function in an animal model. PLoS One, 8, e64726.

Benabid, A. L., Benazzous, A. \& Pollak, P. 2002. Mechanisms of deep brain stimulation. Mov Disord, 17 Suppl 3, S73-4. 
Benabid, A. L., Pollak, P., Louveau, A., Henry, S. \& de Rougemont, J. 1987. Combined (thalamotomy and stimulation) stereotactic surgery of the VIM thalamic nucleus for bilateral Parkinson disease. Appl Neurophysiol, 50, 344-6.

Benabid, A. L., Pollak, P., Seigneuret, E., Hoffmann, D., Gay, E. \& Perret, J. 1993. Chronic VIM thalamic stimulation in Parkinson's disease, essential tremor and extra-pyramidal dyskinesias. Acta Neurochir Suppl (Wien), 58, 39-44.

Benazzouz, A., Breit, S., Koudsie, A., Pollak, P., Krack, P. \& Benabid, A. L. 2002. Intraoperative microrecordings of the subthalamic nucleus in Parkinson's disease. Mov Disord, 17 Suppl 3, S145-9.

Benazzouz, A., Gross, C., Feger, J., Boraud, T. \& Bioulac, B. 1993. Reversal of rigidity and improvement in motor performance by subthalamic high-frequency stimulation in MPTP-treated monkeys. Eur J Neurosci, 5, 382-9.

Benazzouz, A. \& Hallett, M. 2000. Mechanism of action of deep brain stimulation. Neurology, 55, S13-6.

Benazzouz, A., Piallat, B., Pollak, P. \& Benabid, A. L. 1995. Responses of substantia nigra pars reticulata and globus pallidus complex to high frequency stimulation of the subthalamic nucleus in rats: electrophysiological data. Neurosci Lett, 189, 77-80.

Benninger, D. H., Berman, B. D., Houdayer, E., Pal, N., Luckenbaugh, D. A., Schneider, L., Miranda, S. \& Hallett, M. 2011. Intermittent theta-burst transcranial magnetic stimulation for treatment of Parkinson disease. Neurology, 76, 601-9.

Bergman, H., Wichmann, T., Karmon, B. \& DeLong, M. R. 1994. The primate subthalamic nucleus. II. Neuronal activity in the MPTP model of parkinsonism. J Neurophysiol, 72, 507-20.

Boraud, T., Bezard, E., Bioulac, B. \& Gross, C. 1996. High frequency stimulation of the internal Globus Pallidus (GPi) simultaneously improves parkinsonian symptoms and reduces the firing frequency of GPi neurons in the MPTP-treated monkey. Neurosci Lett, $215,17-20$.

Boulet, S., Lacombe, E., Carcenac, C., Feuerstein, C., Sgambato-Faure, V., Poupard, A. \& Savasta, M. 2006. Subthalamic stimulation-induced forelimb dyskinesias are linked to an increase in glutamate levels in the substantia nigra pars reticulata. $J$ Neurosci, 26, 10768-76.

Boyen, K., se Kleine, E., van Dijk, P. \& Langers, D. R. M. 2014. Tinnitus-related dissociation between cortical and subcortical neural activity in humans with mild to moderate sensorineural hearing loss. Hearing Research, 312, 48-59.

Brozoski, T. J. \& Bauer, C. A. 2005. The effect of dorsal cochlear nucleus ablation on tinnitus in rats. Hear Res, 206, 227-36.

Brozoski, T. J., Bauer, C. A. \& Caspary, D. M. 2002. Elevated fusiform cell activity in the dorsal cochlear nucleus of chinchillas with psychophysical evidence of tinnitus. J Neurosci, 22, 2383-90.

Brozoski, T. J., Ciobanu, L. \& Bauer, C. A. 2007. Central neural activity in rats with tinnitus evaluated with manganese-enhanced magnetic resonance imaging (MEMRI). Hear Res, 228, 168-79.

Brozoski, T. J., Wisner, K. W., Sybert, L. T. \& Bauer, C. A. 2011. Bilateral dorsal cochlear nucleus lesions prevent acoustic-trauma induced tinnitus in an animal model. J Assoc Res Otolaryngol, 13, 55-66.

Chang, H., Chen, K., Kaltenbach, J. A., Zhang, J. \& Godfrey, D. A. 2002. Effects of acoustic trauma on dorsal cochlear nucleus neuron activity in slices. Hear Res, 164, 59-68. 
Chen, G. D. \& Jastreboff, P. J. 1995. Salicylate-induced abnormal activity in the inferior colliculus of rats. Hear Res, 82, 158-78.

Chen, G. D., Manohar, S. \& Salvi, R. 2012. Amygdala hyperactivity and tonotopic shift after salicylate exposure. Brain Res.

Cheung, S. W. \& Larson, P. S. 2010. Tinnitus modulation by deep brain stimulation in locus of caudate neurons (area LC). Neuroscience, $169,1768-78$.

Cima, R. F., Maes, I. H., Joore, M. A., Scheyen, D. J., El Refaie, A., Baguley, D. M., Anteunis, L. J., van Breukelen, G. J. \& Vlaeyen, J. W. 2012. Specialised treatment based on cognitive behaviour therapy versus usual care for tinnitus: a randomised controlled trial. Lancet, 379, 1951-9.

Coles, R. R. 1984. Epidemiology of tinnitus: (1) prevalence. Journal of Laryngology \& Otology - Supplement, 9, 7-15.

Day, R. O., Graham, G. G., Bieri, D., Brown, M., Cairns, D., Harris, G., Hounsell, J., Platt-Hepworth, S., Reeve, R., Sambrook, P. N. \& et al. 1989. Concentration-response relationships for salicylate-induced ototoxicity in normal volunteers. Br J Clin Pharmacol, 28, 695-702.

de Koning, P. P., Figee, M., van den Munckhof, P., Schuurman, P. R. \& Denys, D. 2011. Current status of deep brain stimulation for obsessive-compulsive disorder: a clinical review of different targets. Curr Psychiatry Rep, 13, 274-82.

De Ridder, D., De Mulder, G., Verstraeten, E., Seidman, M., Elisevich, K., Sunaert, S., Kovacs, S., Van der Kelen, K., Van de Heyning, P. \& Moller, A. 2007a. Auditory cortex stimulation for tinnitus. Acta Neurochir Suppl, 97, 451-62.

De Ridder, D., Fransen, H., Francois, O., Sunaert, S., Kovacs, S. \& Van De Heyning, P. 2006. Amygdalohippocampal involvement in tinnitus and auditory memory. Acta Otolaryngol Suppl, 50-3.

De Ridder, D., Menovsky, T. \& Van de Heyning, P. 2007b. An otoneurosurgical approach to non-pulsatile and pulsatile tinnitus. B-ENT, 3 Suppl 7, 79-86.

De Ridder, D., Menovsky, T. \& van de Heyning, P. 2008. Auditory cortex stimulation for tinnitus suppression. Otol Neurotol, 29, 574-5; author reply 575 .

De Ridder, D. \& Møller, A. R. 2011. Similarities Between Treatments of Tinnitus and Central Pain. Textbook of Tinnitus.

De Ridder, D., van der Loo, E., Van der Kelen, K., Menovsky, T., van de Heyning, P. \& Moller, A. 2007c. Do tonic and burst TMS modulate the lemniscal and extralemniscal system differentially? Int J Med Sci, 4, 242-6.

De Ridder, D. \& Vanneste, S. 2011. Auditory Cortex Stimulation for Tinnitus. Textbook of Tinnitus.

De Ridder, D., Vanneste, S., Weisz, N., Londero, A., Schlee, W., Elgoyhen, A. B. \& Langguth, B. 2013. An integrative model of auditory phantom perception: Tinnitus as a unified percept of interacting separable subnetworks. Neurosci Biobehav Rev.

Deuschl, G., Schade-Brittinger, C., Krack, P., Volkmann, J., Schafer, H., Botzel, K., Daniels, C., Deutschlander, A., Dillmann, U., Eisner, W., Gruber, D., Hamel, W., Herzog, J., Hilker, R., Klebe, S., Kloss, M., Koy, J., Krause, M., Kupsch, A., Lorenz, D., Lorenzl, S., Mehdorn, H. M., Moringlane, J. R., Oertel, W., Pinsker, M. O., Reichmann, H., Reuss, A., Schneider, G. H., Schnitzler, A., Steude, U., Sturm, V., Timmermann, L., Tronnier, V., Trottenberg, T., Wojtecki, L., Wolf, E., Poewe, W. \& Voges, J. 2006. A randomized trial of deep-brain stimulation for Parkinson's disease. N Engl J Med, 355, 896-908. 
Dong, S., Mulders, W. H., Rodger, J. \& Robertson, D. 2009. Changes in neuronal activity and gene expression in guinea-pig auditory brainstem after unilateral partial hearing loss. Neuroscience, 159, 1164-74.

Dostrovsky, J. O., Levy, R., Wu, J. P., Hutchison, W. D., Tasker, R. R. \& Lozano, A. M. 2000. Microstimulation-induced inhibition of neuronal firing in human globus pallidus. J Neurophysiol, 84, 570-4.

Eggermont, J. J. 2003. Central tinnitus. Auris Nasus Larynx, 30 Suppl, S7-12.

Eggermont, J. J. 2006. Cortical tonotopic map reorganization and its implications for treatment of tinnitus. Acta oto-laryngologica. Supplementum, 9-12.

Eggermont, J. J. 2013. Hearing loss, hyperacusis, or tinnitus: what is modeled in animal research? Hear Res, 295, 140-9.

Engineer, N. D., Riley, J. R., Seale, J. D., Vrana, W. A., Shetake, J. A., Sudanagunta, S. P., Borland, M. S. \& Kilgard, M. P. 2011. Reversing pathological neural activity using targeted plasticity. Nature, 470, 101-4.

Finlayson, P. G. \& Kaltenbach, J. A. 2009. Alterations in the spontaneous discharge patterns of single units in the dorsal cochlear nucleus following intense sound exposure. Hear Res, 256, 104-17.

Fountas, K. N., Kapsalaki, E. \& Hadjigeorgiou, G. 2010. Cerebellar stimulation in the management of medically intractable epilepsy: a systematic and critical review. Neurosurg Focus, 29, E8.

Frank, E., Schecklmann, M., Landgrebe, M., Burger, J., Kreuzer, P., Poeppl, T. B., Kleinjung, T., Hajak, G. \& Langguth, B. 2012. Treatment of chronic tinnitus with repeated sessions of prefrontal transcranial direct current stimulation: outcomes from an open-label pilot study. J Neurol, 259, 327-33.

Frisina, R. D., Walton, J. P. \& Karcich, K. J. 1994. Dorsal cochlear nucleus single neurons can enhance temporal processing capabilities in background noise. Experimental Brain Research, 102, 160-164.

Gardner, G. 1984. Neurologic surgery and tinnitus. J. Laryngol. Otol., Suppl. 9, 311-318.

Gerken, G. M. 1996. Central tinnitus and lateral inhibition: an auditory brainstem model. Hear Res, 97, 75-83.

Grill, W. M., Snyder, A. N. \& Miocinovic, S. 2004. Deep brain stimulation creates an informational lesion of the stimulated nucleus. Neuroreport, 15, 1137-40.

Hamani, C. \& Temel, Y. 2012. Deep brain stimulation for psychiatric disease: contributions and validity of animal models. Sci Trans/ Med, 4, 142 rv8.

Hammond, C., Ammari, R., Bioulac, B. \& Garcia, L. 2008. Latest view on the mechanism of action of deep brain stimulation. Mov Disord, 23, 2111-21.

Hariz, M., Blomstedt, P. \& Zrinzo, L. 2013. Future of brain stimulation: New targets, new indications, new technology. Mov Disord, $28,1784-92$.

Harrison, R. V. 2001. Age-related tonotopic map plasticity in the central auditory pathways. Scand Audiol Suppl, 8-14. 
Hawley, M. L., Melcher, J. R. \& Fullerton, B. C. 2005. Effects of sound bandwidth on fMRI activation in human auditory brainstem nuclei. Hear Res, 204, 101-10.

Heller, A. J. 2003. Classification and epidemiology of tinnitus. Otolaryngologic Clinics of North America, 36, $239-48$.

Hoekstra, C. E., Versnel, H., Neggers, S. F., Niesten, M. E. \& van Zanten, G. A. 2013. Bilateral Low-Frequency Repetitive Transcranial Magnetic Stimulation of the Auditory Cortex in Tinnitus Patients Is Not Effective: A Randomised Controlled Trial. Audiol Neurootol, $18,362-373$.

Holm, A. F., Staal, M. J., Mooij, J. J. \& Albers, F. W. 2005. Neurostimulation as a new treatment for severe tinnitus: a pilot study. Otol Neurotol, 26, 425-8; discussion 428.

House, J. W. \& Brackmann, D. E. 1981. Tinnitus: surgical treatment. Ciba Found Symp, 85, 204-16.

Huang, C. M., Liu, G. \& Huang, R. 1982. Projections from the cochlear nucleus to the cerebellum. Brain Res, 244, 1-8.

Imig, T. J. \& Durham, D. 2005. Effect of unilateral noise exposure on the tonotopic distribution of spontaneous activity in the cochlear nucleus and inferior colliculus in the cortically intact and decorticate rat. J Comp Neurol, 490, 391-413.

Janssen, M. L., Duits, A. A., Tourai, A. M., Ackermans, L., Leentjens, A. F., van Kranen-Mastenbroek, V., Oosterloo, M., Visser-Vandewalle, V. \& Temel, Y. 2014a. Subthalamic nucleus high-frequency stimulation for advanced Parkinson's disease: motor and neuropsychological outcome after 10 years. Stereotact Funct Neurosurg, 92, 381-7.

Janssen, M. L., Zwartjes, D. G., Tan, S. K., Vlamings, R., Jahanshahi, A., Heida, T., Hoogland, G., Steinbusch, H. W., Visser-Vandewalle, V. \& Temel, Y. 2012. Mild dopaminergic lesions are accompanied by robust changes in subthalamic nucleus activity. Neurosci Lett, $508,101-5$.

Janssen, M. L. F., Duits, A. A., Turaihi, A. H., Ackermans, L., Leentjens, A. F. G., van Kranen-Mastenbroek, V., Oosterloo, M., Visser-Vandewalle, V. \& Temel, Y. 2014b. Subthalamic nucleus high frequency stimulation for advanced Parkinson's disease: motor and neuropsychological outcome after 10 years. Stereotact Funct Neurosurg, 92, 381-387.

Jastreboff, P. J. 1990. Phantom auditory perception (tinnitus): mechanisms of generation and perception. Neurosci Res, 8, 221-54.

Jastreboff, P. J. 1999. Tinnitus retraining therapy. Br J Audiol, 33, 68-70.

Jastreboff, P. J. \& Sasaki, C. T. 1994. An animal model of tinnitus: a decade of development. Am J Otol, 15, 19-27.

Jeanmonod, D., Magnin, M. \& Morel, A. 1996. Low-threshold calcium spike bursts in the human thalamus. Common physiopathology for sensory, motor and limbic positive symptoms. Brain, 119 ( Pt 2), 363-75.

Kaltenbach, J. A. 2000. Neurophysiologic mechanisms of tinnitus. J Am Acad Audiol, 11, 125-37.

Kaltenbach, J. A. 2006. Summary of evidence pointing to a role of the dorsal cochlear nucleus in the etiology of tinnitus. Acta Otolaryngol Suppl, 20-6.

Kaltenbach, J. A. 2011. Tinnitus: Models and mechanisms. Hear Res, 276, 52-60. 
Kaltenbach, J. A., Zacharek, M. A., Zhang, J. \& Frederick, S. 2004. Activity in the dorsal cochlear nucleus of hamsters previously tested for tinnitus following intense tone exposure. Neuroscience letters, 355, 121-5.

Kaltenbach, J. A., Zhang, J. \& Finlayson, P. 2005. Tinnitus as a plastic phenomenon and its possible neural underpinnings in the dorsal cochlear nucleus. Hear Res, 206, 200-26.

Kamke, M. R., Brown, M. \& Irvine, D. R. 2003. Plasticity in the tonotopic organization of the medial geniculate body in adult cats following restricted unilateral cochlear lesions. J Comp Neurol, 459, 355-67.

Kandel, E. R., Schwartz, J. H. \& Jessell, T. M. 2000. Principles of neural science, McGraw-Hill, Health Professions Division.

Kim, D. Y., Kim, H. J., Kim, H. I., Oh, H. S., Sim, N. S. \& Moon, I. S. 2014. Long-term Effects of Repetitive Transcranial Magnetic Stimulation in Unilateral Tinnitus. Laryngoscope.

Kleinjung, T., Eichhammer, P., Langguth, B., Jacob, P., Marienhagen, J., Hajak, G., Wolf, S. R. \& Strutz, J. 2005. Long-term effects of repetitive transcranial magnetic stimulation (rTMS) in patients with chronic tinnitus. Otolaryngol Head Neck Surg, 132, 566-9.

Koller, W. C., Lyons, K. E., Wilkinson, S. B., Troster, A. I. \& Pahwa, R. 2001. Long-term safety and efficacy of unilateral deep brain stimulation of the thalamus in essential tremor. Mov Disord, 16, 464-8.

Komiya, H. \& Eggermont, J. J. 2000. Spontaneous firing activity of cortical neurons in adult cats with reorganized tonotopic map following pure-tone trauma. Acta Otolaryngol, 120, 750-6.

Krack, P., Batir, A., Van Blercom, N., Chabardes, S., Fraix, V., Ardouin, C., Koudsie, A., Limousin, P. D., Benazzouz, A., LeBas, J. F., Benabid, A. L. \& Pollak, P. 2003. Five-year follow-up of bilateral stimulation of the subthalamic nucleus in advanced Parkinson's disease. N Engl J Med, 349, 1925-34.

Kraus, K. S. \& Canlon, B. 2012. Neuronal connectivity and interactions between the auditory and limbic systems. Effects of noise and tinnitus. Hear Res.

Kuhn, A. A., Kempf, F., Brucke, C., Gaynor Doyle, L., Martinez-Torres, I., Pogosyan, A., Trottenberg, T., Kupsch, A., Schneider, G. H., Hariz, M. I., Vandenberghe, W., Nuttin, B. \& Brown, P. 2008. High-frequency stimulation of the subthalamic nucleus suppresses oscillatory beta activity in patients with Parkinson's disease in parallel with improvement in motor performance. The Journal of neuroscience : the official journal of the Society for Neuroscience, 28, 6165-73.

Landgrebe, M., Langguth, B., Rosengarth, K., Braun, S., Koch, A., Kleinjung, T., May, A., de Ridder, D. \& Hajak, G. 2009. Structural brain changes in tinnitus: grey matter decrease in auditory and non-auditory brain areas. Neuroimage, 46, 213-8.

Langers, D. R., de Kleine, E. \& van Dijk, P. 2012. Tinnitus does not require macroscopic tonotopic map reorganization. Front Syst Neurosci, 6, 2 .

Langguth, B., de Ridder, D., Dornhoffer, J. L., Eichhammer, P., Folmer, R. L., Frank, E., Fregni, F., Gerloff, C., Khedr, E., Kleinjung, T., Landgrebe, M., Lee, S., Lefaucheur, J. P., Londero, A., Marcondes, R., Moller, A. R., Pascual-Leone, A., Plewnia, C., Rossi, S., Sanchez, T., Sand, P., Schlee, W., Pysch, D., Steffens, T., van de Heyning, P. \& Hajak, G. 2008. Controversy: Does repetitive transcranial magnetic stimulation/transcranial direct current stimulation show efficacy in treating tinnitus patients? Brain Stimul, 1, 192-205.

Langguth, B., Landgrebe, M., Kleinjung, T., Sand, G. P. \& Hajak, G. 2011. Tinnitus and depression. World Journal of Biological Psychiatry, 
$12,489-500$

Lanteaume, L., Khalfa, S., Regis, J., Marquis, P., Chauvel, P. \& Bartolomei, F. 2007. Emotion induction after direct intracerebral stimulations of human amygdala. Cereb Cortex, 17, 1307-13.

Lanting, C. P., De Kleine, E., Bartels, H. \& Van Dijk, P. 2008. Functional imaging of unilateral tinnitus using fMRI. Acta Otolaryngol, $128,415-21$.

Lanting, C. P., de Kleine, E. \& van Dijk, P. 2009. Neural activity underlying tinnitus generation: results from PET and fMRI. Hear Res, 255, 1-13.

Larson, P. S. \& Cheung, S. W. 2012. Deep brain stimulation in area LC controllably triggers auditory phantom percepts. Neurosurgery, 70, 398-405; discussion 405-6.

Larson, P. S. \& Cheung, S. W. 2013. A stroke of silence: tinnitus suppression following placement of a deep brain stimulation electrode with infarction in area LC. J Neurosurg, 118, 192-4.

Leaver, A. M., Renier, L., Chevillet, M. A., Morgan, S., Kim, H. J. \& Rauschecker, J. P. 2011. Dysregulation of limbic and auditory networks in tinnitus. Neuron, 69, 33-43.

Levine, R. A. 1999. Somatic (craniocervical) tinnitus and the dorsal cochlear nucleus hypothesis. Am J Otolaryngol, $20,351-62$.

Lewis, J. E., Stephens, S. D. \& McKenna, L. 1994. Tinnitus and suicide. Clinical Otolaryngology \& Allied Sciences, $19,50-4$.

Lim, H. H., Lenarz, T., Anderson, D. J. \& Lenarz, M. 2008. The auditory midbrain implant: effects of electrode location. Hear Res, 242, 74-85.

Lobarinas, E., Hayes, S. H. \& Allman, B. L. 2012. The gap-startle paradigm for tinnitus screening in animal models: Limitations and optimization. Hear Res, 296, 150-160.

Lobarinas, E., Sun, W., Cushing, R. \& Salvi, R. 2004. A novel behavioral paradigm for assessing tinnitus using schedule-induced polydipsia avoidance conditioning (SIP-AC). Hear Res, 190, 109-14.

Lockwood, A. H., Salvi, R. J., Coad, M. L., Towsley, M. L., Wack, D. S. \& Murphy, B. W. 1998. The functional neuroanatomy of tinnitus: evidence for limbic system links and neural plasticity. Neurology, 50, 114-20.

Lockwood, A. H., Wack, D. S., Burkard, R. F., Coad, M. L., Reyes, S. A., Arnold, S. A. \& Salvi, R. J. 2001. The functional anatomy of gaze-evoked tinnitus and sustained lateral gaze. Neurology, 56, 472-80.

Lowry, L. D., Eisenman, L. M. \& Saunders, J. C. 2004. An absence of tinnitus. Otol Neurotol, 25, 474-8.

Lozano, A. M., Dostrovsky, J., Chen, R. \& Ashby, P. 2002. Deep brain stimulation for Parkinson's disease: disrupting the disruption. Lancet Neurol, 1, 225-31.

Luo, H., Zhang, X., Nation, J., Pace, E., Lepczyk, L. \& Zhang, J. 2012a. Tinnitus suppression by electrical stimulation of the rat dorsal cochlear nucleus. Neurosci Lett, 522, 16-20. 
Luo, H., Zhang, X., Nation, J., Pace, E., Lepczyk, L. \& Zhang, J. 2012b. Tinnitus suppression by electrical stimulation of the rat dorsal cochlear nucleus. Neurosci Lett.

Mahlke, C. \& Wallhäusser-Franke, E. 2004. Evidence for tinnitus-related plasticity in the auditory and limbic system, demonstrated by arg3.1 and c-fos immunocytochemistry. Hear Res, 195, 17-34.

Mallet, N., Ballion, B., Le Moine, C. \& Gonon, F. 2006. Cortical inputs and GABA interneurons imbalance projection neurons in the striatum of parkinsonian rats. J Neurosci, 26, 3875-84.

Manohar, S., Paolone, N. A., Bleichfeld, M., Hayes, S. H., Salvi, R. J. \& Baizer, J. S. 2012. Expression of doublecortin, a neuronal migration protein, in unipolar brush cells of the vestibulocerebellum and dorsal cochlear nucleus of the adult rat. Neuroscience, 202, 169-83.

Manzoor, N. F., Licari, F. G., Klapchar, M., Elkin, R. L., Gao, Y., Chen, G. \& Kaltenbach, J. A. 2012. Noise-induced hyperactivity in the inferior colliculus: its relationship with hyperactivity in the dorsal cochlear nucleus. J Neurophysiol, 108, 976-88.

Martinez Devesa, P., Waddell, A., Perera, R. \& Theodoulou, M. 2007. Cognitive behavioural therapy for tinnitus. Cochrane Database Syst Rev, CD005233.

Matthies, C., Thomas, S., Moshrefi, M., Lesinski-Schiedat, A., Frohne, C., Battmer, R. D., Lenarz, T. \& Samii, M. 2000. Auditory brainstem implants: current neurosurgical experiences and perspective. J Laryngol Otol Suppl, 32-6.

May, B. J. 2000. Role of the dorsal cochlear nucleus in the sound localization behavior of cats. Hear Res, 148, 74-87.

McIntyre, C. C. \& Hahn, P. J. 2010. Network perspectives on the mechanisms of deep brain stimulation. Neurobiol Dis, 38, 329-37.

McIntyre, C. C., Savasta, M., Kerkerian-Le Goff, L. \& Vitek, J. L. 2004. Uncovering the mechanism(s) of action of deep brain stimulation: activation, inhibition, or both. Clin Neurophysiol, 115, 1239-48.

Meikle, M. B., Vernon, J. \& Johnson, R. M. 1984. The perceived severity of tinnitus. Some observations concerning a large population of tinnitus clinic patients. Otolaryngol Head Neck Surg, 92, 689-96.

Meissner, W., Leblois, A., Hansel, D., Bioulac, B., Gross, C. E., Benazzouz, A. \& Boraud, T. 2005. Subthalamic high frequency stimulation resets subthalamic firing and reduces abnormal oscillations. Brain, 128, 2372-82.

Melcher, J. R., Sigalovsky, I. S., Guinan, J. J., Jr. \& Levine, R. A. 2000. Lateralized tinnitus studied with functional magnetic resonance imaging: abnormal inferior colliculus activation. J Neurophysiol, 83, 1058-72.

Middleton, J. W., Kiritani, T., Pedersen, C., Turner, J. G., Shepherd, G. M. \& Tzounopoulos, T. 2011. Mice with behavioral evidence of tinnitus exhibit dorsal cochlear nucleus hyperactivity because of decreased GABAergic inhibition. Proc Natl Acad Sci U S A, 108, 7601-6.

Møller, A. R. 2007. Tinnitus and pain. Prog Brain Res, 166, 47-53.

Møller, A. R. 2011. Epidemiology of Tinnitus in Adults. Textbook of Tinnitus.

Mongan, E., Kelly, P., Nies, K., Porter, W. W. \& Paulus, H. E. 1973. Tinnitus as an indication of therapeutic serum salicylate levels. JAMA, 
$226,142-5$

Montgomery, E. B., Jr. \& Gale, J. T. 2008. Mechanisms of action of deep brain stimulation(DBS). Neurosci Biobehav Rev, 32, $388-407$.

Mühlau, M., Rauschecker, J. P., Oestreicher, E., Gaser, C., Rottinger, M., Wohlschlager, A. M., Simon, F., Etgen, T., Conrad, B. \& Sander, D. 2006. Structural brain changes in tinnitus. Cereb Cortex, 16, 1283-8.

Nondahl, D. M., Cruickshanks, K. J., Huang, G. H., Klein, B. E., Klein, R., Tweed, T. S. \& Zhan, W. 2012. Generational differences in the reporting of tinnitus. Ear \& Hearing, 33, 640-4.

Norena, A. J. 2011. An integrative model of tinnitus based on a central gain controlling neural sensitivity. Neuroscience and biobehavioral reviews, 35, 1089-109.

Norena, A. J. \& Eggermont, J. J. 2003. Changes in spontaneous neural activity immediately after an acoustic trauma: implications for neural correlates of tinnitus. Hear Res, 183, 137-53.

Norena, A. J. \& Eggermont, J. J. 2006. Enriched acoustic environment after noise trauma abolishes neural signs of tinnitus. Neuroreport, 17, 559-63.

O’Donnell, P., Lavin, A., Enquist, L. W., Grace, A. A. \& Card, J. P. 1997. Interconnected parallel circuits between rat nucleus accumbens and thalamus revealed by retrograde transynaptic transport of pseudorabies virus. The Journal of neuroscience : the official journal of the Society for Neuroscience, 17, 2143-67.

Okamoto, H., Stracke, H., Stoll, W. \& Pantev, C. 2010. Listening to tailor-made notched music reduces tinnitus loudness and tinnitus-related auditory cortex activity. Proceedings of the National Academy of Sciences of the United States of America, 107, 1207-10.

Osaki, Y., Nishimura, H., Takasawa, M., Imaizumi, M., Kawashima, T., Iwaki, T., Oku, N., Hashikawa, K., Doi, K., Nishimura, T., Hatazawa, J. \& Kubo, T. 2005. Neural mechanism of residual inhibition of tinnitus in cochlear implant users. Neuroreport, 16, 1625-8.

Paul, A. K., Lobarinas, E., Simmons, R., Wack, D., Luisi, J. C., Spernyak, J., Mazurchuk, R., Abdel-Nabi, H. \& Salvi, R. 2009. Metabolic imaging of rat brain during pharmacologically-induced tinnitus. Neuroimage, 44, 312-8.

Paxinos, G. \& Watson, C. 2007. The Rat Brain in Stereotaxic Coordinates, London, Academic Press.

Petacchi, A., Laird, A. R., Fox, P. T. \& Bower, J. M. 2005. Cerebellum and auditory function: an ALE meta-analysis of functional neuroimaging studies. Hum Brain Mapp, 25, 118-28.

Piccirillo, J. F., Garcia, K. S., Nicklaus, J., Pierce, K., Burton, H., Vlassenko, A. G., Mintun, M., Duddy, D., Kallogjeri, D. \& Spitznagel, E. L., Jr. 2011. Low-frequency repetitive transcranial magnetic stimulation to the temporoparietal junction for tinnitus. Arch Otolaryngol Head Neck Surg, 137, 221-8.

Plewnia, C., Reimold, M., Najib, A., Brehm, B., Reischl, G., Plontke, S. K. \& Gerloff, C. 2007a. Dose-dependent attenuation of auditory phantom perception (tinnitus) by PET-guided repetitive transcranial magnetic stimulation. Hum Brain Mapp, 28, 238-46.

Plewnia, C., Reimold, M., Najib, A., Reischl, G., Plontke, S. K. \& Gerloff, C. 2007b. Moderate therapeutic efficacy of positron emission tomography-navigated repetitive transcranial magnetic stimulation for chronic tinnitus: a randomised, controlled pilot study. $J$ 
Neurol Neurosurg Psychiatry, 78, 152-6.

Rauschecker, J. P. 1999. Auditory cortical plasticity: a comparison with other sensory systems. Trends Neurosci, 22, 74-80.

Rauschecker, J. P., Leaver, A. M. \& Muhlau, M. 2010. Tuning out the noise: limbic-auditory interactions in tinnitus. Neuron, 66, 819-26.

Richardson, D. E. 1967. Thalamotomy for intractable pain. Confin Neurol, 29, 139-45.

Robertson, D., Bester, C., Vogler, D. \& Mulders, W. H. 2013. Spontaneous hyperactivity in the auditory midbrain: relationship to afferent input. Hear Res, 295, 124-9.

Rossi, S., De Capua, A., Ulivelli, M., Bartalini, S., Falzarano, V., Filippone, G. \& Passero, S. 2007. Effects of repetitive transcranial magnetic stimulation on chronic tinnitus: a randomised, crossover, double blind, placebo controlled study. J Neurol Neurosurg Psychiatry, 78, 857-63.

Salvi, R., Wang, H. \& Powers, N. 1996. Rapid functional reorganization in the inferior colliculus and cochlear nucleus after acute cochlear damage. Auditory system plasticity and regeneration, 275-296.

Sedley, W., Teki, S., Kumar, S., Barnes, G. R., Bamiou, D. E. \& Griffiths, T. D. 2012. Single-subject oscillatory gamma responses in tinnitus. Brain.

Seki, S. \& Eggermont, J. J. 2003. Changes in spontaneous firing rate and neural synchrony in cat primary auditory cortex after localized tone-induced hearing loss. Hear Res, 180, 28-38.

Selemon, L. D. \& Goldman-Rakic, P. S. 1985. Longitudinal topography and interdigitation of corticostriatal projections in the rhesus monkey. J Neurosci, 5, 776-94.

Shi, Y., Burchiel, K. J., Anderson, V. C. \& Martin, W. H. 2009. Deep brain stimulation effects in patients with tinnitus. Otolaryngol Head Neck Surg, 141, 285-7.

Shulman, A. \& Strashun, A. 1999. Descending auditory system/cerebellum/tinnitus. Int Tinnitus J, 5, 92-106.

Shulman, A., Strashun, A. M., Afriyie, M., Aronson, F., Abel, W. \& Goldstein, B. 1995. SPECT Imaging of Brain and Tinnitus-Neurotologic/ Neurologic Implications. Int Tinnitus J, 1, 13-29.

Smith, J. A., Mennemeier, M., Bartel, T., Chelette, K. C., Kimbrell, T., Triggs, W. \& Dornhoffer, J. L. 2007. Repetitive transcranial magnetic stimulation for tinnitus: a pilot study. Laryngoscope, 117, 529-34.

Smits, M., Kovacs, S., de Ridder, D., Peeters, R. R., van Hecke, P. \& Sunaert, S. 2007. Lateralization of functional magnetic resonance imaging (fMRI) activation in the auditory pathway of patients with lateralized tinnitus. Neuroradiology, 49, 669-79.

Snider, R. S. \& Stowel, A. 1944. Receiving Areas of the Tactile, Auditory and Visual Systems in the Cerebellum. Journal of Neurophysiology, 7, 331-357.

Soleymani, T., Pieton, D., Pezeshkian, P., Miller, P., Gorgulho, A. A., Pouratian, N. \& De Salles, A. A. 2011. Surgical approaches to tinnitus treatment: A review and novel approaches. Surg Neurol Int, 2, 154. 
Song, J. J., Vanneste, S., Van de Heyning, P. \& De Ridder, D. 2012. Transcranial direct current stimulation in tinnitus patients: a systemic review and meta-analysis. ScientificWorldJournal, 2012, 427941.

Soussi, T. \& Otto, S. R. 1994. Effects of electrical brainstem stimulation on tinnitus. Acta Otolaryngol, 114, 135-40.

Stefani, A., Fedele, E., Galati, S., Pepicelli, O., Frasca, S., Pierantozzi, M., Peppe, A., Brusa, L., Orlacchio, A., Hainsworth, A. H., Gattoni, G., Stanzione, P., Bernardi, G., Raiteri, M. \& Mazzone, P. 2005. Subthalamic stimulation activates internal pallidus: evidence from cGMP microdialysis in PD patients. Ann Neurol, 57, 448-52.

Stolzberg, D., Chen, G. D., Allman, B. L. \& Salvi, R. J. 2011. Salicylate-induced peripheral auditory changes and tonotopic reorganization of auditory cortex. Neuroscience, 180, 157-64.

Su, Y. Y., Luo, B., Jin, Y., Wu, S. H., Lobarinas, E., Salvi, R. J. \& Chen, L. 2012. Altered neuronal intrinsic properties and reduced synaptic transmission of the rat's medial geniculate body in salicylate-induced tinnitus. PLoS One, 7, e46969.

Suneja, S. K., Potashner, S. J. \& Benson, C. G. 1998. Plastic changes in glycine and GABA release and uptake in adult brain stem auditory nuclei after unilateral middle ear ossicle removal and cochlear ablation. Exp Neurol, 151, 273-88.

Tan, S., Vlamings, R., Lim, L., Sesia, T., Janssen, M. L., Steinbusch, H. W., Visser-Vandewalle, V. \& Temel, Y. 2010. Experimental deep brain stimulation in animal models. Neurosurgery, 67, 1073-9; discussion1080.

Temel, Y., Visser-Vandewalle, V., Aendekerk, B., Rutten, B., Tan, S., Scholtissen, B., Schmitz, C., Blokland, A. \& Steinbusch, H. W. 2005. Acute and separate modulation of motor and cognitive performance in parkinsonian rats by bilateral stimulation of the subthalamic nucleus. Exp Neurol, 193, 43-52.

Turner, J. G., Brozoski, T. J., Bauer, C. A., Parrish, J. L., Myers, K., Hughes, L. F. \& Caspary, D. M. 2006. Gap detection deficits in rats with tinnitus: a potential novel screening tool. Behav Neurosci, 120, 188-95.

Tyler, R. S. 2012. Patient preferences and willingness to pay for tinnitus treatments. J Am Acad Audiol, 23, 115-25.

Tziridis, K., Ahlf, S. \& Schulze, H. 2012. A low cost setup for behavioral audiometry in rodents. J Vis Exp.

Tziridis, K., Korn, S., Ahlf, S. \& Schulze, H. 2014. Protective Effects of Ginkgo biloba Extract EGb 761 against Noise Trauma-Induced Hearing Loss and Tinnitus Development. Neural Plast, In press.

Tzounopoulos, T. 2008. Mechanisms of synaptic plasticity in the dorsal cochlear nucleus: plasticity-induced changes that could underlie tinnitus. Am J Audiol, 17, S170-5.

Van de Heyning, P., Vermeire, K., Diebl, M., Nopp, P., Anderson, I. \& De Ridder, D. 2008. Incapacitating unilateral tinnitus in single-sided deafness treated by cochlear implantation. Ann Otol Rhinol Laryngol, 117, 645-52.

van Gendt, M. J., Boyen, K., de Kleine, E., Langers, D. R. \& van Dijk, P. 2012. The relation between perception and brain activity in gaze-evoked tinnitus. J Neurosci, 32, 17528-39.

Vanneste, S., Plazier, M., der Loo, E., de Heyning, P. V., Congedo, M. \& De Ridder, D. 2010. The neural correlates of tinnitus-related distress. Neuroimage, 52, 470-80. 
Vitek, J. L. 2002. Mechanisms of deep brain stimulation: excitation or inhibition. Mov Disord, 17 Suppl 3, S69-72.

Vogler, D. P., Robertson, D. \& Mulders, W. H. 2011. Hyperactivity in the ventral cochlear nucleus after cochlear trauma. J Neurosci, 31, 6639-45.

Wallhäusser-Franke, E., Mahlke, C., Oliva, R., Braun, S., Wenz, G. \& Langner, G. 2003. Expression of c-fos in auditory and non-auditory brain regions of the gerbil after manipulations that induce tinnitus. Exp Brain Res, 153, 649-54.

Wang, H., Brozoski, T. J., Turner, J. G., Ling, L., Parrish, J. L., Hughes, L. F. \& Caspary, D. M. 2009. Plasticity at glycinergic synapses in dorsal cochlear nucleus of rats with behavioral evidence of tinnitus. Neuroscience, 164, 747-59.

Wang, J., Ding, D. \& Salvi, R. J. 2002. Functional reorganization in chinchilla inferior colliculus associated with chronic and acute cochlear damage. Hear Res, 168, 238-49.

Wickesberg, R. E. \& Oertel, D. 1990. Delayed, frequency-specific inhibition in the cochlear nuclei of mice: a mechanism for monaural echo suppression. J Neurosci, 10, 1762-8.

Windels, F., Bruet, N., Poupard, A., Feuerstein, C., Bertrand, A. \& Savasta, M. 2003. Influence of the frequency parameter on extracellular glutamate and gamma-aminobutyric acid in substantia nigra and globus pallidus during electrical stimulation of subthalamic nucleus in rats. J Neurosci Res, 72, 259-67.

Windels, F., Carcenac, C., Poupard, A. \& Savasta, M. 2005. Pallidal origin of GABA release within the substantia nigra pars reticulata during high-frequency stimulation of the subthalamic nucleus. J Neurosci, 25, 5079-86.

Yang, S., Weiner, B. D., Zhang, L. S., Cho, S. J. \& Bao, S. 2011. Homeostatic plasticity drives tinnitus perception in an animal model. Proc Natl Acad Sci U S A, 108, 14974-9.

Yeterian, E. H. \& Pandya, D. N. 1998. Corticostriatal connections of the superior temporal region in rhesus monkeys. J Comp Neurol, 399, 384-402.

Zeng, C., Nannapaneni, N., Zhou, J., Hughes, L. F. \& Shore, S. 2009. Cochlear damage changes the distribution of vesicular glutamate transporters associated with auditory and nonauditory inputs to the cochlear nucleus. J Neurosci, 29, 4210-7.

Zhang, J. \& Zhang, X. 2010. Electrical stimulation of the dorsal cochlear nucleus induces hearing in rats. Brain Res, 1311, 37-50.

Zoger, S., Svedlund, J. \& Holgers, K. M. 2006. Relationship between tinnitus severity and psychiatric disorders. Psychosomatics, 47, 282-8. 


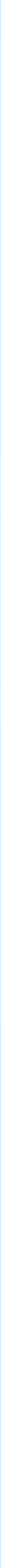




\section{Bhanter 3 The impact of deep brain stimulation on timnitus}

JV Smit, MLF Janssen, M Engelhard, RMA de Bie, PR Schuurman, MF Contarino, A Mosch,

Y Temel, RJ Stokroos

Surgical neurology international 7 (2016) S848-54 


\section{Abstract}

Tinnitus is a disorder of the nervous system which cannot be adequately treated with current therapies. The effect of neuromodulation induced by deep brain stimulation (DBS) on tinnitus has not been studied well. This study investigated the effect of DBS on tinnitus by use of a multicenter questionnaire study.

Tinnitus was retrospectively assessed prior to DBS and at the current situation (with DBS). From the 685 questionnaires, 443 were returned. A control group was one-to-one matched to DBS patients who had tinnitus before DBS ( $n=61$ ). Tinnitus was assessed by the tinnitus handicap inventory (THI) and visual analogue scales (VAS) of loudness and burden.

The THI decreased significantly during DBS compared to the situation prior to surgery (from 18.9 to 15.1, p<.001), which was only significant for DBS in the subthalamic nucleus (STN). The THI in the control group (36.9 to $35.5, p=.50$ ) and other DBS targets did not change. The VAS loudness increased in the control group (5.4 to $6.0 p<.01$ ).

DBS might have a modulatory effect on tinnitus. Our study suggests that DBS of the STN may have a beneficial effect on tinnitus, but most likely other nuclei linked to the tinnitus circuitry might be even more effective. 


\section{Introduction}

Tinnitus, also known as ringing in the ears, is defined as a perception of sound when no actual external sound is present. The prevalence of frequent and burdensome tinnitus is $10-15 \%$ and increased over the last decennia (Nondahl et al., 2012). Tinnitus can be associated with psychiatric disorders, such as anxiety and depression. The lifetime risk of a major depression in people with disabling tinnitus is $78 \%$ compared to $21 \%$ in the normal population (Sullivan et al., 1988). Despite the large impact on the daily life of patients and the substantial economic burden on the society, there is still no satisfactory treatment available that attenuates tinnitus.

It is thought that tinnitus is formed following a plasticity-related overcompensation of the central nervous system resulting in a pathological neuronal activity in auditory and non-auditory networks (Kaltenbach, 2011, Eggermont, 2003, Norena, 2011).

Deep brain stimulation can be applied within neuronal networks to reduce the pathological neuronal activity (McIntyre and Hahn, 2010). There is more and more evidence that deep brain stimulation (DBS) is able to attenuate tinnitus (Smit et al., 2015, van Zwieten et al., 2016). Both animal and human studies have shown reduction in the loudness of tinnitus by targeting the dorsal cochlear nucleus (Luo et al., 2012), the ventral intermediate nucleus of the thalamus (VIM )(Shi et al., 2009) and area LC of the caudate nucleus (Cheung and Larson, 2010) with DBS. DBS is already frequently and successfully applied for treatment-resistant patients with Parkinson's disease (PD), essential tremor (ET) and dystonia and good outcomes have been demonstrated for epilepsy, obsessive-compulsive disorder and Tourette syndrome. The subthalamic nucleus (STN), VIM and globus pallidus internus (GPi) are common targets for DBS. However, it is not yet clear if there is a modulatory effect from DBS on tinnitus.

Our goal was to assess severity of tinnitus before and during DBS in patients who were treated with DBS in a broad spectrum of targets and for various indications. For this purpose, we conducted a multicenter survey study in a large DBS patient cohort. We carefully matched this group with a control cohort that contained tinnitus patients without DBS. 


\title{
Materials and methods
}

\author{
Patient characteristics
}

All patients who underwent DBS surgery in the Maastricht University Medical Center, Amsterdam Medical Center or the Haga Teaching Hospital (The Hague), were included in this study and received a questionnaire by mail. The first part of the questionnaire consisted of questions about the situation before DBS surgery $(t=0)$ and the second part consisted of questions about the current situation with $\operatorname{DBS}(\mathrm{t}=1)$.

After patient characteristics of the DBS patients were analyzed, we gathered a control group by advertisements among patients known to have tinnitus to retrospectively examine the natural course of tinnitus over time. These tinnitus patients were asked to fill out the same questionnaire online, which consisted of questions about the situation 4 years ago $(t=0)$ and the current situation $(t=1)$. The 4 -year interval was based on the mean time between DBS surgery and completion of the questionnaire in the DBS group, which was $3.9 \pm 2.9$ years. Control patients were matched with the 61 DBS patients who declared to have had tinnitus before surgery, in a matched subject design for the dichotomous variables gender, tinnitus treatment (e.g. medication or cognitive therapy) and self-reported hearing loss ('yes, measured by audiometry', 'yes, noticed by my relatives or myself', 'no'). From the available matched controls, the control subjects that matched age as best were chosen. Informed consents were obtained from all patients. No ethical approval was required for this type of questionnaire study according to local regulations.

\section{Study design}

Both parts of the questionnaire included general questions about tinnitus (duration, received treatment), hearing loss (objective or subjective) and three tinnitus severity measures. The main tinnitus outcome measure was the tinnitus handicap inventory (THI), which consists of 25 questions and reflects the impact of tinnitus on daily living. For each item or situation, subjects were asked to fill in one of the following responses: 'yes' (4 points), 'sometimes' (2 points), or 'no' (0 points), resulting in a maximum burden score of 100. Furthermore, the questionnaire included a visual analogue scale (VAS) of tinnitus loudness ( 0 is no loudness and 10 is being the most loud tinnitus) and a VAS of burden ( 0 is no burden and 10 is the most burdening tinnitus).

For all measures the absolute mean at $\mathrm{t}=0$ and $\mathrm{t}=1$ was calculated for the within-group comparisons. Because of the baseline differences between the DBS and control group, between-group comparison was based on the mean of the relative change in percentage from each subject, expressed as where $x$ is an outcome measure from a single subject. Subjects who forgot to fill in some questions were included in the study but statistical analysis could only be performed for questions that were answered in both situations. For this reason, the population size might differ among different measures. In all 
cases, analysis in the control group was only performed for the one-to-one matched subjects.

In the group of patients that only experienced tinnitus after DBS surgery, the 5-year incidence was calculated and compared to the literature.

\section{Statistics}

We used a Student's t-test for normally distributed data and the Wilcoxon signed rank test for nonparametric data. The within-subjects comparisons were analyzed in a paired manner. P-values less than 0.05 were considered statistically significant. All data were analyzed by using SPSS (Version 20, IBM, Somers, NY, USA). 


\section{Results}

\section{Patient characteristics}

Questionnaires were sent to 685 DBS patients. Six patients or their relatives wrote down that they were not able to fill out the form because of mental or physical problems. A return rate of filled out questionnaires of $65 \%(n=443)$ was achieved.

From the responders, 61 (14\%) had tinnitus before DBS (group 1), 61 (14\%) had newly formed tinnitus following DBS surgery (group 2) and 328 DBS patients (72\%) did not experience tinnitus at all (group 3) (see Figure 1). The control group (group $4, n=61$ ) was drawn from a group of 613 subjects and was precision-matched to group 1 (see Table 1). From the patients with PD, 77\% had electrodes implanted in the STN, $12 \%$ in the VIM and $11 \%$ in the GPi.
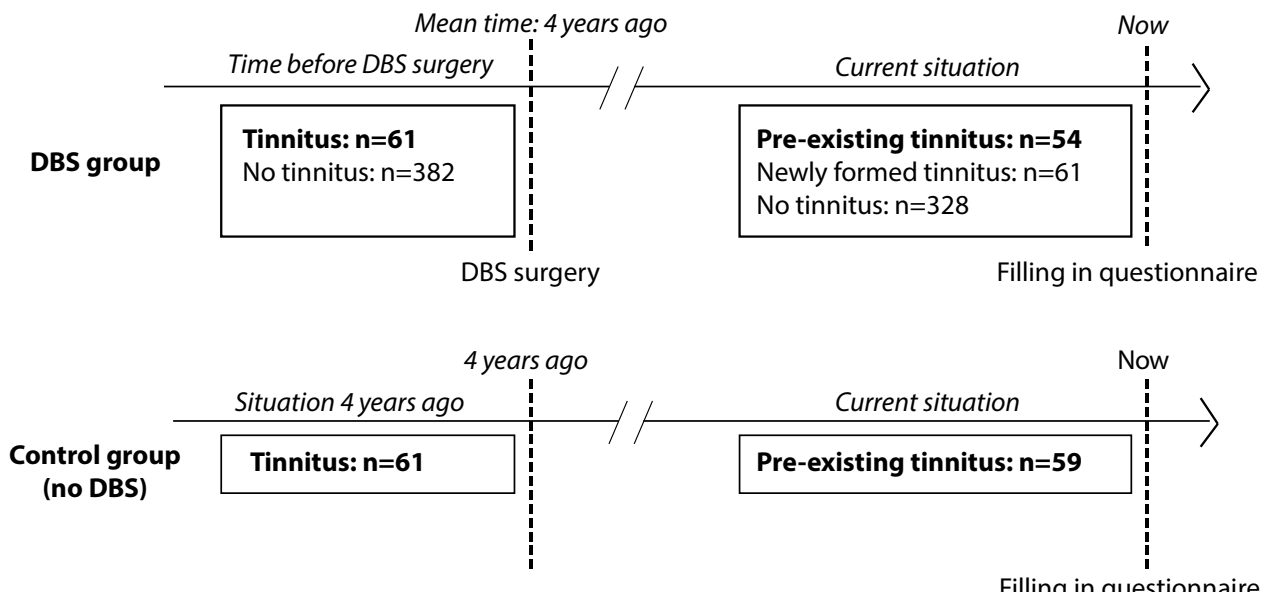

Filling in questionnaire

Figure 1: Scheme of the study design. DBS patients were asked to fill in a questionnaire about the situation before DBS (mean time 3.9 years ago) and about the current situation. In a matched subject design, the control group was matched to those patients of the DBS group who had pre-existing tinnitus. Patients from the control group were first asked to fill in a tinnitus questionnaire regarding their situation 4 years ago (similar to the situation of the DBS group). Second, the patients were asked to fill in the questionnaire for the current situation. Note that 7 patients who had tinnitus prior to DBS surgery did not have tinnitus during DBS. In the control group, 2 patients only experienced tinnitus 4 years ago. DBS = deep brain stimulation. 
Table 1: Characteristics of patients with pre-existing tinnitus before DBS (1), newly formed tinnitus during DBS (2), no tinnitus (3) and a control group with pre-existing tinnitus (4), that was matched to group 1. Matching variables were age, gender, hearing loss and treatment. Other targets (last row) include combinations of subthalamic nucleus and ventral intermediate nucleus of the thalamus, nucleus accumbens and posterior hypothalamus.

\begin{tabular}{|c|c|c|c|c|}
\hline & \multicolumn{3}{|c|}{ DBS } & \multirow{2}{*}{$\begin{array}{l}\text { Control } \\
\text { 4) Pre-existing } \\
\text { tinnitus ( } n=61)\end{array}$} \\
\hline & $\begin{array}{l}\text { 1) Pre-existing } \\
\text { tinnitus ( } n=61)\end{array}$ & $\begin{array}{l}\text { 2) Newly formed } \\
\text { tinnitus ( } n=61)\end{array}$ & $\begin{array}{l}\text { 3) No tinnitus } \\
\text { (n=328) }\end{array}$ & \\
\hline Age (years, mean \pm SEM, range) & $64.3 \pm 11.5(23-82)$ & $64.1 \pm 11.4(41-90)$ & $64.1 \pm 10.9(22-85)$ & $63.3 \pm 10.2(30-83)$ \\
\hline Man/woman (\%/\%) & $64 / 36$ & $67 / 33$ & $60 / 40$ & $64 / 36$ \\
\hline Hearing loss (\%) & 48 & 44 & 12 & 48 \\
\hline Hearing loss objectified (\%) & 69 & 56 & 60 & 45 \\
\hline Tinnitus duration (years, mean \pm SEM, range) & $17.7 \pm 14.9(1-57)$ & $4.40 \pm 3.4(0-15)$ & - & $15.0 \pm 12.6(4-71)$ \\
\hline Treatment for tinnitus (\%) & 11 & 4 & - & 11 \\
\hline Bilateral stimulation (\%) & 90 & 87 & 92 & - \\
\hline \multicolumn{5}{|l|}{ Primary indication for DBS (\%) } \\
\hline - Parkinson's disease & 67 & 64 & 76 & \\
\hline - Essential tremor & 19 & 17 & 9 & \\
\hline - Obsessive compulsive disorder & 3 & 2 & 2 & \\
\hline - Tourette syndrome & 2 & 7 & 1 & \\
\hline - Dystonia & 7 & 5 & 10 & \\
\hline - Multiple sclerosis & 2 & 2 & 1 & \\
\hline - Postischemic tremor & 0 & 1 & 1 & \\
\hline - Epilepsia & 0 & 0 & 1 & \\
\hline - Painsyndrome & 0 & 0 & 0.3 & \\
\hline \multicolumn{5}{|l|}{ DBS target } \\
\hline - Subthalamic nucleus & 52 & 44 & 60 & \\
\hline - Ventral intermediate nucleus of the thalamus & 26 & 32 & 18 & \\
\hline - Globus pallidus internus & 19 & 10 & 18 & \\
\hline - Subthalamic area (zona inserta) & 2 & 5 & 1 & \\
\hline $\begin{array}{l}\text { - Centromedian nucleus, substantia } \\
\text { periventricularis, and nucleus ventrooralis } \\
\text { internus }\end{array}$ & 0 & 7 & 1 & \\
\hline - Other targets & 2 & 2 & 3 & \\
\hline
\end{tabular}

$D B S=$ deep brain stimulation, $S E M=$ standard error of the mean

\section{Tinnitus outcome}

From the 61 patients who experienced tinnitus before DBS surgery, 7 were completely tinnitus-free at the time of the questionnaire. The THI decreased significantly compared to the situation before DBS surgery (from 18.9 to 15.1 ) whereas the THI in the control group did not significantly change (36.9 to 35.5). With respect to the THI in the DBS group, $53 \%$ improved, $36 \%$ did not change and $11 \%$ worsened. 
In the control group 54\% improved, 7\% did not change and 39\% worsened.

For the patients with DBS, the reported loudness and burden on a VAS-scale did not change significantly (respectively 3.9 to 3.9 and 3.7 to 3.5). The control group showed a significant increase in VAS loudness (5.4 to 6.0) and a non-significant increase in VAS burden (4.9 to 5.3). Detailed results with $p$-values are shown in Table 2.

Table 2: The THI, VAS loudness and VAS burden of tinnitus before $(t=0)$ and during DBS ( $t=1)$. Measurements were performed for all DBS patients and in subgroup analyses that consisted of only patients with electrodes implanted in the STN, VIM or GPi. Note that the control group did not receive DBS. The mean is shown, with the standard error of the mean between brackets.

\begin{tabular}{l|llllllllll}
\multirow{2}{*}{ Groups } & \multicolumn{4}{c}{ THI } & \multicolumn{3}{c}{ VAS loudness } & \multicolumn{3}{c}{ VAS burden } \\
\cline { 2 - 11 } & $\mathbf{t = 0}$ & $\mathbf{t}=\mathbf{1}$ & $\boldsymbol{p}$-value & $\mathbf{t}=\mathbf{0}$ & $\mathbf{t}=\mathbf{1}$ & $\boldsymbol{p}$-value & $\mathbf{t = 0}$ & $\mathbf{t}=\mathbf{1}$ & $\boldsymbol{p}$-value \\
\hline DBS, all targets & $18.9(2.5)$ & $15.1(2.6)$ & $<.001(n=53)$ & $3.9(0.3)$ & $3.9(0.4)$ & $p=.95(n=53)$ & $3.7(0.3)$ & $3.5(0.4)$ & $.69(n=50)$ \\
STN & $18.7(3.3)$ & $14.7(3.1)$ & $<.01(n=30)$ & $3.8(0.4)$ & $3.9(0.5)$ & $p=.79(n=31)$ & $3.4(0.5)$ & $3.3(0.5)$ & $.77(n=30)$ \\
VIM & $28.1(6.2)$ & $18.1(6.0)$ & $.11(n=14)$ & $4.8(0.4)$ & $4.5(0.6)$ & $p=.58(n=13)$ & $4.5(0.4)$ & $4.8(0.6)$ & $<.05(n=13)$ \\
GPi & $13.6(5.4)$ & $12.5(7.3)$ & $.35(n=8)$ & $3.4(0.5)$ & $3.1(0.9)$ & $p=.60(n=8)$ & $3.6(0.6)$ & $3.0(0.9)$ & $.35(n=8)$ \\
Control & $36.9(3.7)$ & $35.5(3.7)$ & $.55(n=53)$ & $5.4(0.4)$ & $6.0(0.4)$ & $p=<.01(n=53)$ & $4.9(0.4)$ & $5.3(0.4)$ & $.20(n=50)$ \\
\hline
\end{tabular}

$T H I=$ tinnitus handicap inventory, VAS = visual analog scale, DBS = deep brain stimulation, STN = subthalamic nucleus, VIM = ventral intermediate nucleus of the thalamus, GPi = globus pallidus internus.

Relative measures were used to compare the DBS group to the control group. The relative mean difference of the THI was $-19 \%$ for the DBS group and $+14 \%$ for the control group. In respectively the DBS and control group, VAS loudness increased with 13\% and 35\% and VAS burden increased with 21\% and $38 \%$. Relative measures of the three outcome measures are visualized in Figure 2.

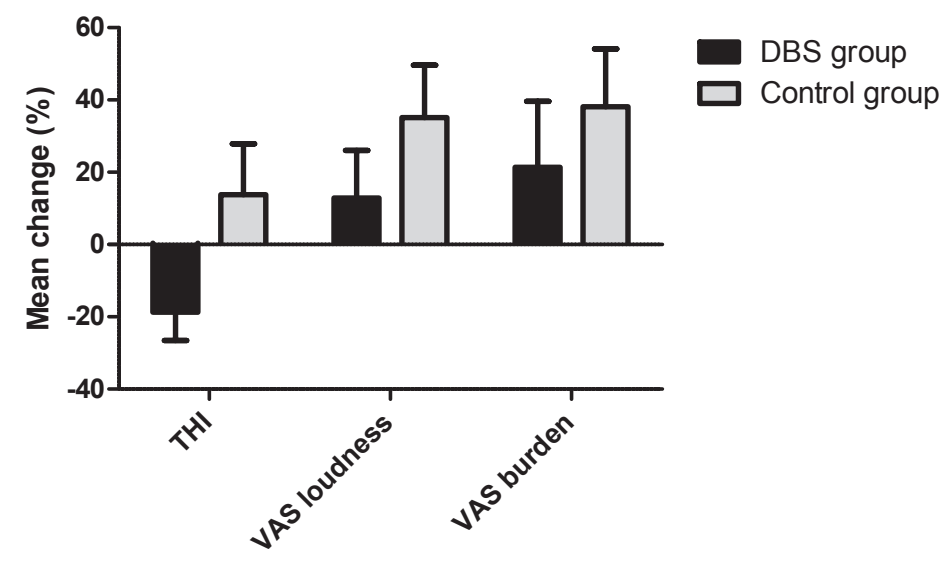

Figure 2: The mean of the relative change (in percentage) is calculated for the THI, VAS loudness and VAS burden. A positive number indicates an increase and a negative number a decrease in the outcome. Error bars indicate the absolute standard error of the mean. $D B S=$ deep brain stimulation, $T H I=$ tinnitus handicap inventory, VAS = visual analog scale 


\section{Subgroup analyses}

For the DBS group with existing tinnitus, the mean values of the THI were calculated for the most common targets: STN $(n=31)$, the VIM $(n=17)$ and the GPi $(n=11)$. The STN was the only target that showed a statistically significant decrease in THI (18.7 to 14.7), see Table 2 for more extensive results.

The primary indication for DBS, which is not independent from the target subgroup analysis, was subdivided in PD, ET and others. When analyzing the subgroups for primary indication, only in the group with PD a significant difference in mean THI difference was seen (18.6 to $14.8, p<.01, \mathrm{n}=34$ ).

Comparing males and females, only males showed a significant difference in THI (20.1 to 15.4, p<.001, $\mathrm{n}=34$ ). Females did not show a significant difference (16.7 to $14.6, p=.26, \mathrm{n}=19$ ).

Both subjects who received treatment for tinnitus (e.g. medication or cognitive therapy), as subjects who did not receive treatment for tinnitus showed a significant decrease of THI (17.6 to 14.2, $p<.01$, $\mathrm{n}=40$ and 35.4 to $27.4, p<.05, \mathrm{n}=7$, respectively).

The only subgroup analysis that showed a significant difference in a VAS scale was VIM as a DBS target; VAS loudness increased from 4.5 to $4.7(p<.05, n=13)$.

\section{Newly formed tinnitus}

The 5-year incidence of tinnitus is based on the percentage of subjects who developed tinnitus after DBS. From the 200 patients with 5 year follow-up, 21 developed tinnitus after DBS surgery, all with STN as the target. The 5 -year incidence is $10.5 \%$. From all subjects who developed tinnitus following DBS, 6 patients did not fill in the onset date of tinnitus, and were excluded from analysis. 


\section{Discussion}

The present study showed that the THI reduced significantly in patients with DBS, while it did not significantly change in the control group that represented the natural course of tinnitus over time. In the patients who received DBS, the relative change of the THI was a decrease of $19 \%$, whereas the relative change in the control group was an increase of $14 \%$. During DBS there was no change on the VAS loudness and burden, while the VAS loudness increased significantly in the control group.

The results from the subgroup analysis showed that the STN, which is the most common DBS target for $\mathrm{PD}$, is the only target that reached statistical significance in the within-subject comparison of the THI. The VIM showed a large decrease of the THI (28.1 to 18.1), which might not have reached significance due to the small sample size $(n=14)$. The heterogeneity of the study population could lead to an underestimation of the effectiveness of DBS on tinnitus.

It was not our purpose in this study to assess the clinical relevance of performing DBS for tinnitus. In this study, tinnitus was assessed in patients who did not seek for treatment of their tinnitus by way of DBS. Therefore, the baseline THI is relatively low and therefore a clinical relevant decrease of 7 points on the THI (Landgrebe et al., 2012, Zeman et al., 2011) is not a realistic outcome measure. Studies which will prospectively assess the clinical relevance of DBS as a treatment for tinnitus should perform DBS with tinnitus as the primary indication and should carefully select appropriate and highly suffering tinnitus patients. Furthermore, the target to be stimulated with DBS should be selected with care.

To the best of our knowledge, the effect of STN stimulation on tinnitus has not been assessed before. The influence on tinnitus by VIM stimulation has been reported in another study (Martin et al., 1999). In that study, out of 29 patients with VIM DBS for PD or ET, 7 reported to have tinnitus. Three patients reported that their tinnitus was quieter during DBS and four patients reported that DBS had no effect on tinnitus. Two patients who noted a decrease in tinnitus during DBS and two patients who did not note a reduction were evaluated in the clinic where the stimulator was turned off and on. Tinnitus characterization and test-retest evaluation with DBS turned on and off showed the same outcome as the patients reported via the questionnaire. This points out the reliability of retrospective patients-reported self-evaluation of the presence and severity of tinnitus. In our study we found a trend towards a reduction of tinnitus on the THI by VIM stimulation.

As far as we know, no other study retrospectively or prospectively assessed the natural course of tinnitus over time with absolute values of tinnitus severity. Some studies only presented the presence of tinnitus (Rosenhall and Karlsson, 1991, Stouffer and Tyler, 1990, Rubenstein et al., 1992) or tinnitus grades (Davis and El Rafaie, 2000, Nondahl et al., 2002, Andersson et al., 2001) over time. The only statistical comparison that was conducted in a long-term follow-up, expressed tinnitus distress levels in three different grades using the Klockhoff and Lindblom rating scale, and showed that the tinnitus severity 
deteriorated but not significantly, which is in accordance to our results (Andersson et al., 2001). We showed that the THI remained unchanged after 4 years of follow-up (36.9 to 35.5, $p=.55$ ). Furthermore, the VAS loudness increased significantly (5.4 to 6.0) and VAS burden increased non-significantly (4.9 to 5.3 ).

DBS has good therapeutic effects in PD and ET, both on motor outcome and the quality of life. The effect of DBS on tinnitus could be explained by interference with the tinnitus-related neuronal network. In the present study, the STN showed the best outcome in tinnitus reduction. The STN, which is not directly linked to auditory nuclei, is subdivided in a motor, associative and limbic part. The STN however, is connected to the nucleus accumbens, which has been implicated to play a role in tinnitus (Smit et al., 2015). The effect of STN stimulation might be explained by this afferent connection. Another explanation could be that STN DBS patients with improved tinnitus had a perioperative focal lesion of the caudate nucleus due to the traversing lead. It has been demonstrated in a case report that infarction of the caudate nucleus due to an electrode in STN DBS surgery could lead to a complete suppression of tinnitus (Larson and Cheung, 2013). This finding has been reevaluated in a study where the electrode is paused in area LC of caudate nucleus, which is the area of the caudate that is traversed during DBS STN surgery. From the 6 patients that were evaluated, 5 patients indicated tinnitus suppression during stimulation of the area LC of the caudate nucleus. The authors suggested that the dorsal striatum acts as a gate to control loudness of tinnitus (Cheung and Larson, 2010). The gate theory explains that phantom sounds could be generated by DBS (Larson and Cheung, 2012). This was demonstrated by intra-operative stimulation of the caudate nucleus in PD patients. Electrical stimulation of the caudate nucleus induced sounds in patients with and without tinnitus (Larson and Cheung, 2012). This is an important finding in further unravelling the pathophysiology of tinnitus.

Although only one case study reported tinnitus as a side effect of DBS (in the VIM) (Ondo et al., 2001), our data showed that the 5 -year incidence of tinnitus in the DBS population is $10.5 \%$, which is almost twice as high as the previously reported 5-year tinnitus incidence of 5.7\% (Nondahl et al., 2002). Despite that our study setup was not primarily designed for assessing the incidence of tinnitus, the higher incidence suggest that DBS might also generate tinnitus in a certain number of patients.

One might conclude that the STN is a potential target to treat tinnitus by DBS. This conclusion however needs to be tempered. The targets investigated by this study are clinically used as a target for other primary indications than tinnitus. For this reason, it seems reasonable to think that stimulation of brain areas that are more directly involved in the neural network involved in tinnitus will be more effective. One of the promising targets is the dorsal cochlear nucleus. Stimulation of this target has already been shown to attenuate tinnitus in an animal study (Luo et al., 2012). Other potential targets in the auditory network, which are directly related to tinnitus, are the inferior colliculus and the medial geniculate body of the thalamus. Non-auditory regions are involved in tinnitus and should therefore also be considered as targets for DBS include the cerebellum, amygdala, hippocampus and nucleus accumbens (Smit et al., 2015). 
The main limitation of this is study is the retrospective design of the questionnaire which could be prone to 'recall bias'. It could be difficult to remember tinnitus characteristics from the past and patients may tend to have a better recall on past exposures than controls (Coughlin, 1990). However, the advantage of this study design, also known as 'then-test', is the absence of a 'response shift'. Tinnitus can only be assessed by subjective measurements. Alterations of these subjective measures can be the result of an objective change such as a treatment, but may also be the result of changes in their internal standards, values and conceptualization of quality of life when a change in health is experienced (Schwartz et al., 2006). In other words, if similar questions are asked at different time points, people tend to answer differently due to different psychometric properties related to their altered health situation. For example in Ménière's disease, response shift has been demonstrated following adaption of this chronic illness (Yardley and Dibb, 2007). In case of asking retrospective and current question at the same time, this potential bias does not occur. A similar then-test design has recently been used to retrospectively study the effect of cochlear implantation on tinnitus (Kloostra et al., 2015).

Furthermore, to strengthen the design of the study, we made use of a control group. To match the DBS patients as good as possible with the controls, we used a one-to-one matching strategy with multiple matching variables. The control group consisted of subjects from the general population who suffered from tinnitus. Despite that some studies find an abnormal hearing in PD and ET patients (Yylmaz et al., 2009, Ondo et al., 2003, Vitale et al., 2012) other studies found normal hearing in these patients (Chiappa, 1997, Fradis et al., 1988, Prasher and Bannister, 1986). To our knowledge, a correlation of $\mathrm{PD} / \mathrm{ET}$ with tinnitus has never been reported. Hence, the control group seems to be appropriate for this study to compare the course of time with the DBS group. However, because the groups were not matched for disease and baseline tinnitus characteristics, we did not try to statistically compare the two groups with each other. Finally, the results presented should be interpreted with caution. It might be well possible that the tinnitus handicap reported by patients decreased due to an improved of quality of life by the effect of DBS on their motor symptoms.

This study is one of the first steps in exploring the feasibility and target specificity of DBS as a future treatment for tinnitus. The present study indicates that DBS might reduce the handicap that is caused due to tinnitus. Furthermore, while the tinnitus loudness remained the same in the DBS group, it increased in the control group. Stimulation of the STN resulted in the most beneficial effect on tinnitus, but stimulation of other nuclei that are directly linked to the tinnitus circuitry might be even more effective. 


\section{References}

Andersson, G., Vretblad, P., Larsen, H. C. \& Lyttkens, L. 2001. Longitudinal follow-up of tinnitus complaints. Arch Otolaryngol Head Neck Surg, 127, 175-9.

Cheung, S. W. \& Larson, P. S. 2010. Tinnitus modulation by deep brain stimulation in locus of caudate neurons (area LC). Neuroscience, $169,1768-78$

Chiappa, K. H. 1997. Short-latency somatosensory evoked potentials: interpretation. In: CHIAPPA, K. H. (ed.) Evoked Potentials in Clinical Medicine. 3rd ed. New York: Lippincott-Raven.

Coughlin, S. S. 1990. Recall bias in epidemiologic studies. J Clin Epidemiol, 43, 87-91.

Davis, A. \& El Rafaie, A. 2000. Epidemiology of tinnitus. In: TYLER, R. S. (ed.) Tinnitus Handbook. San Diego: Singular.

Eggermont, J. J. 2003. Central tinnitus. Auris Nasus Larynx, 30 Suppl, S7-12.

Fradis, M., Samet, A., Ben-David, J., Podoshin, L., Sharf, B., Wajsbort, J., Zellinger, M. \& Pratt, H. 1988. Brainstem auditory evoked potentials to different stimulus rates in parkinsonian patients. Eur Neurol, 28, 181-6.

Kaltenbach, J. A. 2011. Tinnitus: Models and mechanisms. Hear Res, 276, 52-60.

Kloostra, F. J., Arnold, R., Hofman, R. \& Van Dijk, P. 2015. Changes in tinnitus after cochlear implantation and its relation with psychological functioning. Audiol Neurootol, 20, 81-9.

Landgrebe, M., Azevedo, A., Baguley, D., Bauer, C., Cacace, A., Coelho, C., Dornhoffer, J., Figueiredo, R., Flor, H., Hajak, G., van de Heyning, P., Hiller, W., Khedr, E., Kleinjung, T., Koller, M., Lainez, J. M., Londero, A., Martin, W. H., Mennemeier, M., Piccirillo, J., De Ridder, D., Rupprecht, R., Searchfield, G., Vanneste, S., Zeman, F. \& Langguth, B. 2012. Methodological aspects of clinical trials in tinnitus: a proposal for an international standard. J Psychosom Res, 73, 112-21.

Larson, P. S. \& Cheung, S. W. 2012. Deep brain stimulation in area LC controllably triggers auditory phantom percepts. Neurosurgery, 70, 398-405; discussion 405-6.

Larson, P. S. \& Cheung, S. W. 2013. A stroke of silence: tinnitus suppression following placement of a deep brain stimulation electrode with infarction in area LC. J Neurosurg, 118, 192-4.

Luo, H., Zhang, X., Nation, J., Pace, E., Lepczyk, L. \& Zhang, J. 2012. Tinnitus suppression by electrical stimulation of the rat dorsal cochlear nucleus. Neurosci Lett, 522, 16-20.

Martin, W. H., Shi, Y.-B., Buciel, K. J. \& Anderson, V. C. 1999. Deep brain stimulation effects on hearing function and tinnitus. Sixth International Tinnitus Seminar.

McIntyre, C. C. \& Hahn, P. J. 2010. Network perspectives on the mechanisms of deep brain stimulation. Neurobiol Dis, 38, 329-37.

Nondahl, D. M., Cruickshanks, K. J., Huang, G. H., Klein, B. E., Klein, R., Tweed, T. S. \& Zhan, W. 2012. Generational differences in the reporting of tinnitus. Ear Hear, 33, 640-4. 
Nondahl, D. M., Cruickshanks, K. J., Wiley, T. L., Klein, R., Klein, B. E. \& Tweed, T. S. 2002. Prevalence and 5-year incidence of tinnitus among older adults: the epidemiology of hearing loss study. J Am Acad Audiol, 13, 323-31.

Norena, A. J. 2011. An integrative model of tinnitus based on a central gain controlling neural sensitivity. Neuroscience and biobehavioral reviews, 35, 1089-109.

Ondo, W., Dat Vuong, K., Almaguer, M., Jankovic, J. \& Simpson, R. K. 2001. Thalamic deep brain stimulation: effects on the nontarget limbs. Mov Disord, 16, 1137-42.

Ondo, W. G., Sutton, L., Dat Vuong, K., Lai, D. \& Jankovic, J. 2003. Hearing impairment in essential tremor. Neurology, 61, 1093-7.

Prasher, D. \& Bannister, R. 1986. Brain stem auditory evoked potentials in patients with multiple system atrophy with progressive autonomic failure (Shy-Drager syndrome). J Neurol Neurosurg Psychiatry, 49, 278-89.

Rosenhall, U. \& Karlsson, A. K. 1991. Tinnitus in old age. Scand Audiol, 20, 165-71.

Rubenstein, B., T., Ö. \& Rosenhall, U. 1992. Longitudinal fluctuations in tinnitus as reported by an elderly population. J Audiol Med, $1,149-155$

Schwartz, C. E., Bode, R., Repucci, N., Becker, J., Sprangers, M. A. \& Fayers, P. M. 2006. The clinical significance of adaptation to changing health: a meta-analysis of response shift. Qual Life Res, 15, 1533-50.

Shi, Y., Burchiel, K. J., Anderson, V. C. \& Martin, W. H. 2009. Deep brain stimulation effects in patients with tinnitus. Otolaryngol Head Neck Surg, 141, 285-7.

Smit, J. V., Janssen, M. L., Schulze, H., Jahanshahi, A., Van Overbeeke, J. J., Temel, Y. \& Stokroos, R. J. 2015. Deep brain stimulation in tinnitus: Current and future perspectives. Brain Res, 1608, 51-65.

Stouffer, J. L. \& Tyler, R. S. 1990. Characterization of tinnitus by tinnitus patients. J Speech Hear Disord, 55, 439-53.

Sullivan, M. D., Katon, W., Dobie, R., Sakai, C., Russo, J. \& Harrop-Griffiths, J. 1988. Disabling tinnitus. Association with affective disorder. Gen Hosp Psychiatry, 10, 285-91.

van Zwieten, G., Smit, J. V., Jahanshahi, A., Temel, Y. \& Stokroos, R. J. 2016. Tinnitus: Is there a place for brain stimulation? Surg Neurol Int, 10, S125-9.

Vitale, C., Marcelli, V., Allocca, R., Santangelo, G., Riccardi, P., Erro, R., Amboni, M., Pellecchia, M. T., Cozzolino, A., Longo, K., Picillo, M., Moccia, M., Agosti, V., Sorrentino, G., Cavaliere, M., Marciano, E. \& Barone, P. 2012. Hearing impairment in Parkinson's disease: expanding the nonmotor phenotype. Mov Disord, 27, 1530-5.

Yardley, L. \& Dibb, B. 2007. Assessing subjective change in chronic illness: An examination of response shift in health-related and goal-oriented subjective status. Psychology and Health, 22, 813-828.

Yylmaz, S., Karaly, E., Tokmak, A., Guclu, E., Kocer, A. \& Ozturk, O. 2009. Auditory evaluation in Parkinsonian patients. Eur Arch Otorhinolaryngol, 266, 669-71.

Zeman, F., Koller, M., Figueiredo, R., Aazevedo, A., Rates, M., Coelho, C., Kleinjung, T., de Ridder, D., Langguth, B. \& Landgrebe, M. 
2011. Tinnitus handicap inventory for evaluating treatment effects: which changes are clinically relevant? Otolaryngol Head Neck Surg, 145, 282-7. 


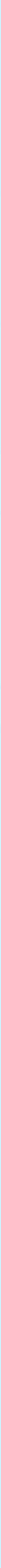




\section{Bhanter 4}

\section{Patient acceptance of invasive treatments for tinnitus}




\section{Abstract}

The field of neuromodulation is currently seeking to treat a wide range of disorders with various types of invasive devices. In recent years several preclinical trials and case reports in humans have been published on their potential for chronic tinnitus. However, studies to obtain insight in patients' willingness to undergo these treatments are scarce. The aim of this survey study was to find out whether tinnitus patients are willing to undergo invasive neuromodulation when taking its risks, costs and potential benefits into account.

A visual analogue scale (VAS, 0-10) was used to measure the outcome. Spearman's rank-order correlation coefficients were computed to determine the correlation between patient characteristics and acceptance rates.

Around one-fifth of the patients were reasonably willing to undergo invasive treatment (VAS 5-7), and around one-fifth were fully willing to do so (VAS 8-10). Hearing aids, used as a control, were accepted most, followed by cochlear implantation, deep brain stimulation and cortical stimulation. Acceptance rates were slightly higher when the chance of cure was higher. Patients with a history of attempted treatments were more eager than others to find a new treatment for tinnitus.

A considerable proportion of tinnitus patients would accept a variety of invasive treatments despite the associated risks or costs. When clinical neuromodulatory studies for tinnitus are to be performed, particular attention should be given to obtaining informed consent, including explaining the potential risks and providing a realistic outcome expectation. 


\section{Introduction}

Tinnitus is the perception of sound in the absence of an external acoustic stimulus. The prevalence of tinnitus in the global population is 10 to $15 \%$ (Baguley et al., 2013). Its burden can lead to a severe decrease in the quality of life among 1 to 2\% of the population (Langguth et al., 2013). As recently estimated, tinnitus-related health care cost per patient is $€ 1544$ per year; overall, tinnitus-related health care costs account for approximately $2.3 \%$ of health care expenditure in the Netherlands (Maes et al., 2013).

Several therapies are currently available for tinnitus patients, mainly counseling and psycho-education (Jastreboff and Jastreboff, 2000, Martinez-Devesa et al., 2010, Hesser et al., 2011). While these therapies may improve the quality of life, they do not diminish the actual perception of sound.

The absence of a treatment that silences the sound may be partly due to the complex pathophysiology of tinnitus. While the exact pathology remains unclear, it is presumed that pathological central neuronal activity forms the basis for tinnitus (Henry et al., 2014). The current hypothesis is that noise trauma can damage the cochlear hair cells. The resulting eighth nerve deafferentation leads to a central gain upregulation thereby increasing spontaneous activity, bursting activity and neural synchrony of the central auditory system (Eggermont and Roberts, 2004, Norena and Farley, 2013, Kaltenbach, 2011, Chen et al., 2015).

Currently, new treatments are being developed to interfere with this central pathological neuronal activity. Neuromodulation can act at cochlear level (cochlear implantation, $\mathrm{Cl}$ ), on deep brain structures (deep brain stimulation, DBS) or at cortical level (cortical stimulation, CS). $\mathrm{Cl}$ and CS are already being used experimentally in humans with tinnitus as the primary indication (Mertens et al., 2016, Arts et al., 2015, De Ridder et al., 2006, Smit et al., 2015). Preclinical studies for the treatment of tinnitus using DBS have shown promising results (Smit et al., 2016b, Luo et al., 2012). In humans, tinnitus has been reduced in patients with advanced Parkinson's disease who have been treated with DBS (Shi et al., 2009, Cheung and Larson, 2010, Smit et al., 2016a).

The above treatments require invasive surgical procedures with their associated risks and costs. At this stage of development, insight in patients' preferences for invasive treatment options - including the acceptance of possible side effects, the risks of potential complications and the willingness to pay for a treatment-could eventually lead to therapies with a high socio-economic acceptance. To this end we developed and carried out a survey to assess the willingness of patients to undergo invasive treatments for tinnitus. 


\section{Methods}

\section{Patient characteristics}

In November 2014 an internet survey was conducted among tinnitus patients who were members of Dutch Society of Hard of Hearing People. All participants suffered from tinnitus and were 18 years or older. The surgical procedure and its potential risks were explained using written text and illustrations (see Appendix).

Questions related to patient characteristics included age, sex, the loudness and burden of tinnitus (using a Visual Analogue Scale (VAS) from 0 to 10) and number of attempted treatments. To objectify the burden, the survey used the Mini-Tinnitus Questionnaire (Mini-TQ), which is validated in a Dutch population (Vanneste et al., 2011). The Mini-TQ has 12 items and a maximum score of 24 (Hiller and Goebel, 2004). Psychological health was assessed with the Patient Health Questionnaire (PHQ-9), a nine-item depression scale with a maximum score of 27, validated in Dutch (Zuithoff et al., 2010).

\section{Outcome measurements}

The primary outcome was the willingness to undergo treatment if the success rate is either $50 \%$ or $100 \%$. To explore the acceptance of $\mathrm{Cl}, \mathrm{DBS}$ and $\mathrm{CS}$, non-invasive therapy with HA was taken as reference. On a VAS ranging from 0 to 10 , a score of 0 meant 'I would not accept this treatment at all' and 10 'I would fully accept this treatment'. For the descriptive analysis, the scores were divided into 3 groups: 'no acceptance' (VAS 0-4), 'reasonable acceptance' (VAS 5-7) and 'full acceptance' (VAS 8-10).

There were several secondary outcome measurements: acceptance of side effects, deafness and death; the amount of money patients are willing to pay for the treatment; and correlations of patient characteristics with acceptance of treatments. Furthermore, the acceptance rate was compared between patients who have had therapies for tinnitus versus patients without a history of previous therapies. Side effects were classified as 'mild' (i.e. temporary or slightly bothersome) and 'severe' (i.e. chronic or bothersome). The answer options were stated as 'I would not take that risk', '0.1\% chance', ' $1 \%$ chance', ' $10 \%$ chance', ' $20 \%$ chance' and ' $50 \%$ chance'. Options regarding the willingness to pay for treatment were 'less than $1 / 4$ of my monthly income', ' $1 / 4$ of my monthly income', ' $1 / 2$ of my monthly income', 'my monthly income', 'twice my monthly income', '5 times my monthly income', '10 times my monthly income', '20 times my monthly income' and 'over 20 times my monthly income'.

\section{Statistics}

The statistical analysis was performed with IBM SPSS Statistics version 22. Data was presented as mean \pm standard deviation. Based on visual inspection, kurtosis and skewness, it was determined that the primary and secondary outcome data were not normally distributed. A Mann-Whitney $U$ test was performed for an independent two-group comparison. Multiple related groups were compared with 
a Friedman test; where statistically significant effects were identified, post-hoc comparisons were performed with a two-tailed Wilcoxon rank-sum test. Spearman rank-order correlations were used to identify correlations between variables. An alpha value of 0.05 was considered significant with a Bonferroni adjustment for multiple comparisons.

Ethics

No ethical approval was required for this type of study according to the Dutch Central Committee on Research Involving Human Subjects (CCMO). All subjects included in the study population declared no objection to the use of data for medical research.

\section{Results}

\section{Patient demographics}

The questionnaire was filled out by 415 patients, whose demographics are shown in Table 1. Figure 1 displays boxplots of the tinnitus scores (VAS loudness, VAS burden and Mini-TQ).

Table 1. Patient demographics

\begin{tabular}{ll} 
Variable & Outcome \\
\hline Men/women $(n / n)$ & $252 / 163$ \\
Age in years (mean $\pm S D$, range) & $58 \pm 12,18-64$ \\
Patients suffering from hearing loss $(n)$ & 380 \\
Tinnitus duration in years (mean $\pm S D$, range) & $15 \pm 13,1-60$ \\
Days per month aware of tinnitus (mean $\pm S D$, range) & $28 \pm 7,3-31$ \\
Percentage of the day aware of tinnitus (mean $\pm S D$, range) & $79 \pm 31,0-100$ \\
VAS tinnitus loudness (mean $\pm S D$, range) & $6.1 \pm 2.2,0-10$ \\
VAS tinnitus burden (mean $\pm S D$, range) & $5.6 \pm 2.7,0-10$ \\
Mini-TQ score (mean $\pm S D$, range) & $11.0 \pm 5.8,0-23$ \\
PHQ-9 score (mean $\pm S D$, range) & $6.9 \pm 5.4,0-27$ \\
Number of attempted treatments for tinnitus (mean $\pm S D$, range) & $0.91 \pm 1.1,0-4$ \\
\hline
\end{tabular}

Mini-TQ $=$ Mini-Tinnitus Questionnaire, $P H Q-9=$ Patient Health Questionnaire-9, SD =Standard Deviation, VAS = Visual Analogue Scale 

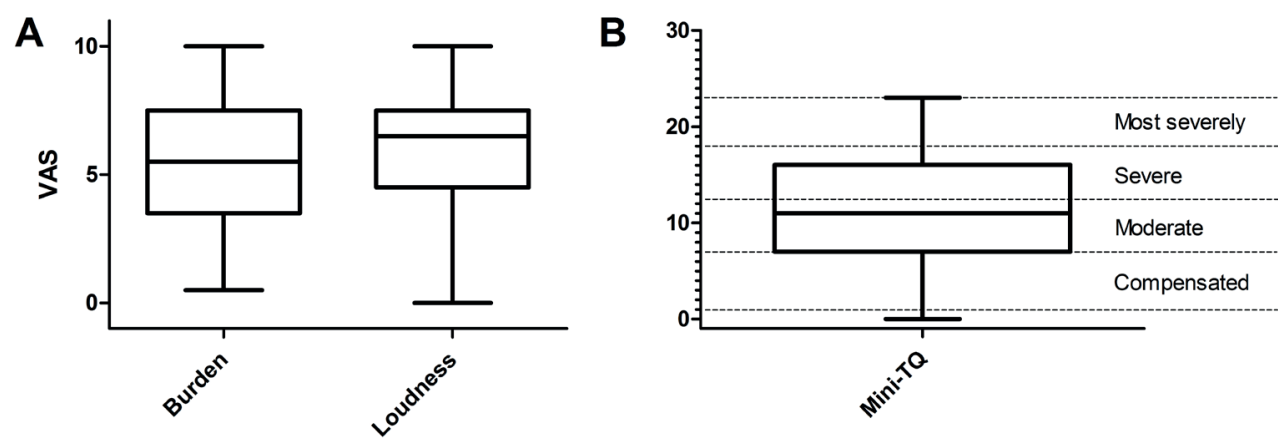

Figure 1: A. Boxplots of VAS burden (0-10), VAS loudness (0-10) and B. Mini-TQ (0-27), categorized under compensated (1-7), moderately distressed (8-12), severely distressed (13-18), most severely distressed (19-24).

\section{Acceptance rates}

The mean VAS acceptance is depicted in Figure 2a. To give a more descriptive picture, the acceptance rate is subdivided into no acceptance, reasonable acceptance and full acceptance, as shown in Figure $2 b$.

A

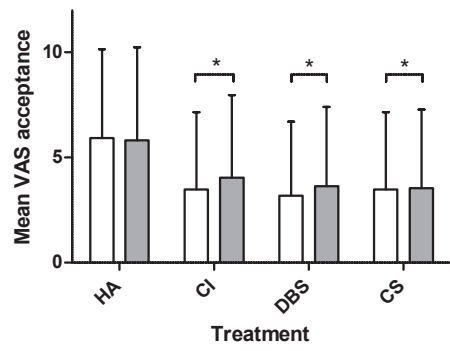

$\square 50 \%$ chance of tinnitus cure $\square 100 \%$ chance of tinnitus cure

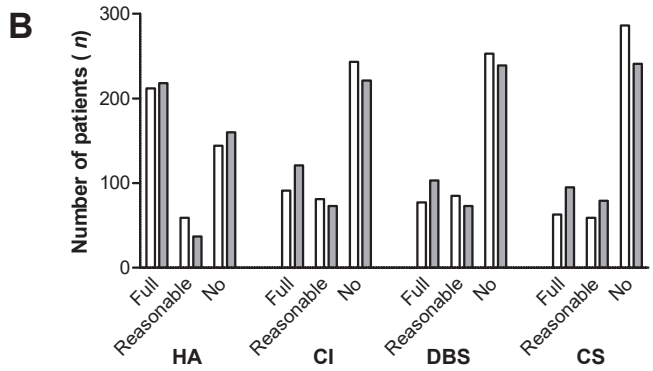

Subdivided acceptance rates per treatment

Figure 2. A. Mean acceptance VAS scores. An asterisk indicates a significant difference between $50 \%$ and $100 \%$ chance of cure (p $<0.0125$ ) calculated with the Wilcoxon signed rank test. For $50 \%$ and $100 \%$ chance of cure, the Friedman test showed a significant difference in VAS acceptance depending on the type of treatment (not illustrated in the figure, $p<0.0083$ ). B. Group acceptance of tinnitus treatment. The total number of patients is 415 . 'Full' is defined as a VAS score $\geq 8$, 'reasonable' 4-7 and 'no' $\leq 3$. White bars indicate a treatment success chance of $50 \%$ and gray bars of $100 \%$. $\mathrm{HA}=$ hearing aid, $\mathrm{Cl}=$ cochlear implant, $\mathrm{DBS}=$ deep brain stimulation, CS=cortical stimulation

Where there is a $50 \%$ chance of cure with $\mathrm{HA}$ and where that chance is $100 \%$, the rates of full acceptance were respectively $51 \%$ and $53 \%$, rates of reasonable acceptance were $14 \%$ and $9 \%$ and rates of no acceptance were $35 \%$ and $39 \%$. In case of a $50 \%$ chance of cure with $\mathrm{Cl}$ and a $100 \%$ chance, full acceptance rates were respectively $22 \%$ and $29 \%$, reasonable acceptance rates were $20 \%$ and $18 \%$ and 
no acceptance rates were $59 \%$ and $53 \%$. For a $50 \%$ and $100 \%$ chance of cure, DBS was fully accepted by respectively $19 \%$ and $25 \%$, reasonably accepted by $21 \%$ and $18 \%$ and not accepted by $61 \%$ and $58 \%$ of the respondents. In case of a 50\% chance of cure and a 100\% chance of cure, CS was fully accepted by respectively $15 \%$ and $23 \%$, reasonably accepted by $20 \%$ and $19 \%$ and not accepted by $65 \%$ and $58 \%$.

With a $50 \%$ chance of cure, the Friedman test showed a significant difference in VAS acceptance depending on the type of treatment $\left(X^{2}(3)=210.368, p<.0005\right)$. The post-hoc Wilcoxon test showed a significant difference between all treatments with the exception of DBS and $\mathrm{Cl}$ (CS vs $\mathrm{Cl} Z=-4.539, p<$ 0.0005 ; DBS vs Cl $Z=-2.344, p=0.019$; HA vs $\mathrm{Cl} Z=-9.997, p<0.0005$; DBS vs CS $Z=-2.659, p=0.008$; HA vs CS $Z=-11.061, p<0.0005$; HA vs DBS $Z=-10.046, p<0.0005)$. A Bonferonni adjusted alpha level of 0.0083 was used.

Also in case of a $100 \%$ chance of cure, the Friedman test showed a significant difference in VAS acceptance depending on the type of treatment $\left(X^{2}(3)=190.642, p<.0005\right)$. The post-hoc Wilcoxon test showed a significant difference between all treatments except for DBS and $\mathrm{Cl}$ (CS vS Cl $Z=-4.158$, $p<0.0005$; DBS vS Cl $Z=-3.210, p<0.005 ; \mathrm{HA}$ vs Cl $Z=-8.124, p<0.0005$; DBS vs CS $Z=-.879, p=0.380$; HA vs CS $Z=-9.269, p<0.0005$; HA vs DBS $Z=-8.597, p<0.0005)$. A Bonferonni adjusted alpha level of 0.0083 was used.

There was a significant difference in acceptance rate between a $50 \%$ and a $100 \%$ chance of cure for DBS $(Z=-4.639, p=.00), \mathrm{Cl}(Z=-4.681, p=.00)$ and $\mathrm{CS}(Z=-5.418, p<.0005)$. HA was not significant $(Z=-.198$, $p=.843)$. An alpha level of 0.0125 was used for the Bonferonni adjustment.

\section{Potential side effects}

The willingness to accept a risk (0.1-50\%) of mild and severe side effects was respectively $68 \%$ and $48 \%$ in case of a $50 \%$ chance of remission and $72 \%$ and $55 \%$ in case of a $100 \%$ chance of remission. Deafness and death were accepted as side effects by respectively $33 \%$ and $19 \%$ in case of a $50 \%$ chance of remission and $36 \%$ and $20 \%$ in case of a $100 \%$ chance of remission. The chance of these potential side effects is further categorized as $0.1 \%, 1 \%, 10 \%, 20 \%$ and $50 \%$ (see Figure 3 ). 


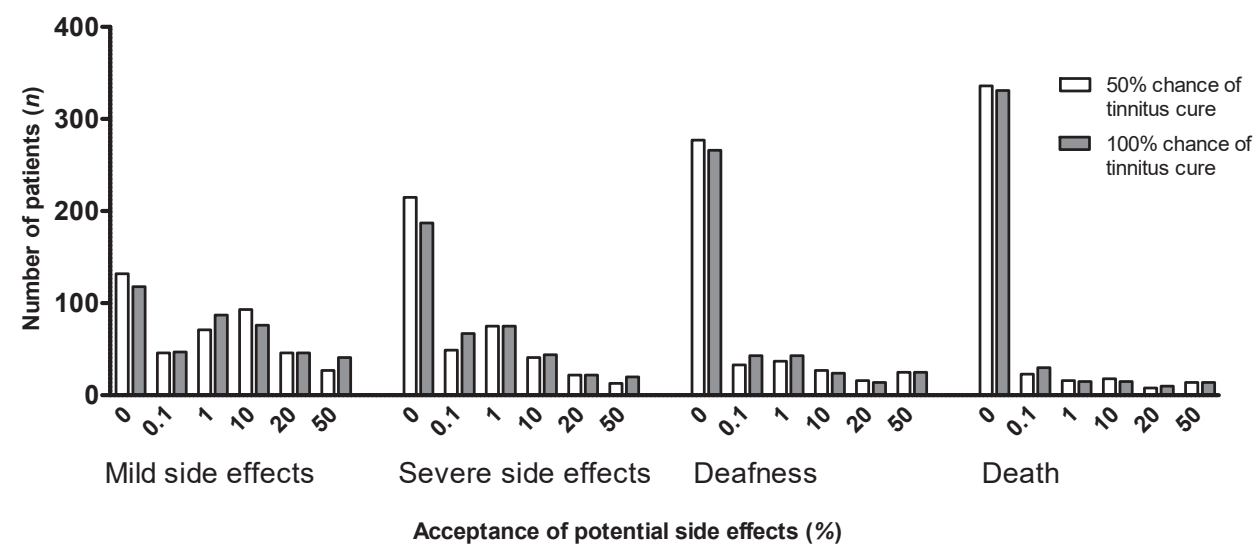

Figure 3. Group acceptance of potential side effects divided into mild side effects, severe side effects, deafness and death. The total number of patients is 415 . White bars indicate a treatment success chance of $50 \%$ and gray bars of $100 \%$.

Costs

Figure 4 depicts willingness to pay for a treatment that cures tinnitus. The group of patients who were willing to pay 20 times their monthly income or more reported higher VAS loudness than the rest of the study sample ( $7.3 \pm 2.0$ vs. $5.6 \pm 2.2, Z=-6.960, p<.0005)$, a higher VAS burden ( $7.3 \pm 2.2$ vs. $4.9 \pm$ $2.5, Z=-8.298, p<.0005)$, a higher mean Mini-TQ score (14.9 \pm 5.0 vs. $9.9 \pm 5.5, Z=-7.864, p<.0005)$, a higher PHQ-9 score $(9.6 \pm 6.0$ vs. $5.8 \pm 4.7, Z=-6.318, p<.0005)$ and a lower age ( $55.7 \pm 10.1$ vs. 59.3 \pm 12.2 year, $Z=-3.824, p<.0005)$. The difference in tinnitus duration ( $15.8 \pm 13.0$ vs. $15.2 \pm 12.3, Z=$ $-.155, p=.877)$, days of tinnitus awareness per month $(28.1 \pm 7.1 \pm 28.6 \pm 6.6, Z=-.550, p=0.583)$ and percentage of tinnitus awareness per day $(86.6 \pm 23.7 \pm 75.4 \pm 33.0, Z=-2.606, p=0.009)$ were not statistically significant, according to a Bonferonni adjusted alpha level of 0.006 . 


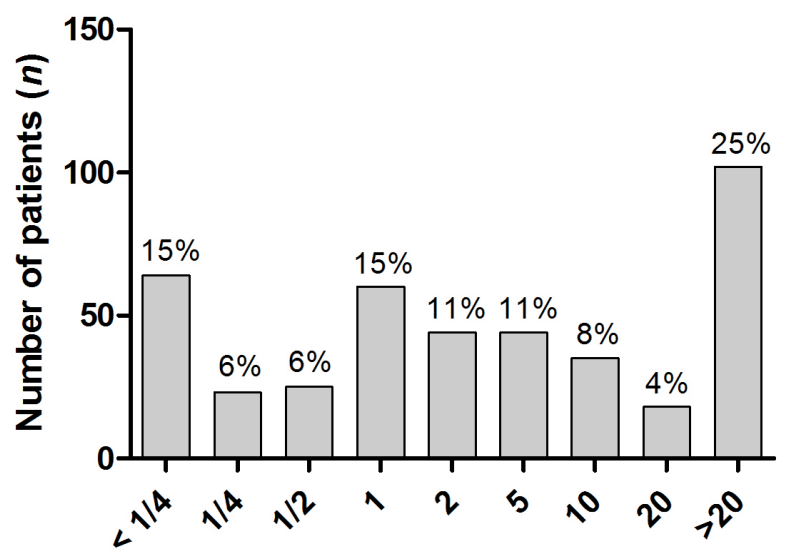

Willingness to pay ( ${ }^{*}$ times monthly income)

Figure 4. Willingness of patients to pay for treatment that cures their tinnitus. The amount of money is related to their monthly income.

Subgroup analysis

Correlations between acceptance rates and patient characteristics were calculated to assess whether certain characteristics contributed to treatment acceptance. For all invasive treatments, significant correlations with acceptance were found for loudness, burden, percentage of tinnitus awareness per day, mini-TQ and PHQ-9. Age was only related to acceptance in case of a 100\% chance of cure. All correlations were weak.

Correlations were also computed between acceptance rates and risks. Only for invasive treatments moderate correlations were found between acceptance and mild side effects, severe side effects and deafness. Weaker correlations were found for death (see Table 2 for Spearman's $r$ values). 


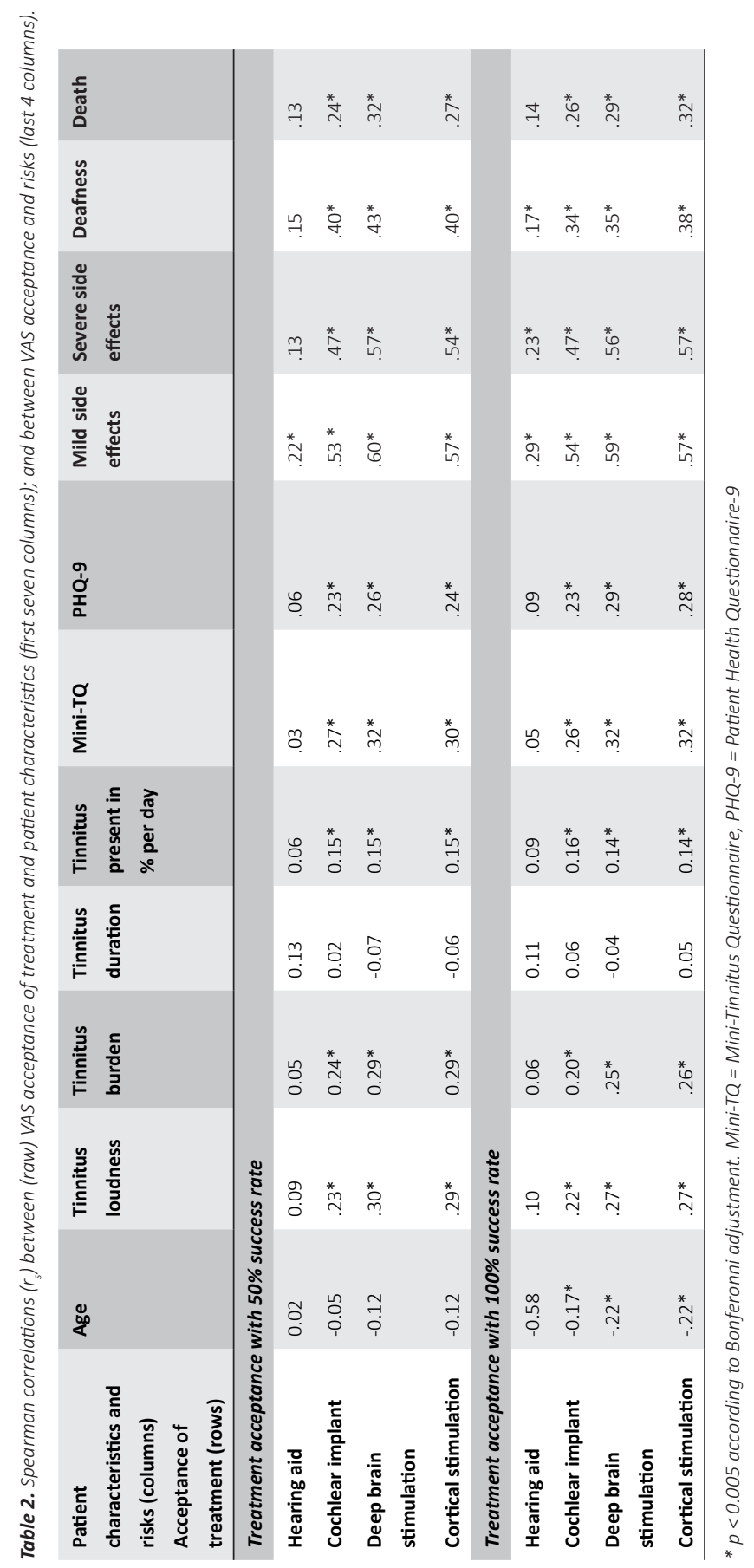


The acceptance rates for a 50\% and 100\% chance of eliminating tinnitus were compared for patients with and without a history of attempted tinnitus treatments (Table 3 ). For a $50 \%$ chance of cure, there was a significant difference for HA $(Z=-2.64, p=0.008)$, DBS $(Z=-3.59, p<0.005)$ and $\mathrm{CS}(Z=-3.37, p=$ $0.001)$. For a $100 \%$ chance of cure, there was a significant difference for $\operatorname{DBS}(Z=3.38, p=0.001)$ and CS $(Z=-3.28, p=0.001)$.

Table 3. Mean VAS acceptance rates in patients with and without a history of tinnitus treatments. Mean acceptance rates (VAS) are shown with the standard deviation. A Mann-Whitney $U$ test was performed. The Bonferonni adjusted alpha-value was 0.0125.

\begin{tabular}{|c|c|c|c|c|}
\hline Chance of cure & Treatment & $\begin{array}{l}\text { History without treatments } \\
\text { (acceptance rate, } \mathrm{n}=\mathbf{2 0 0} \text { ) }\end{array}$ & $\begin{array}{l}\text { History with } \geq 1 \text { tinnitus treatments } \\
\text { (acceptance rate, } n=215 \text { ) }\end{array}$ & $P$-value \\
\hline \multirow[t]{4}{*}{$50 \%$} & Hearing aid & $5.3 \pm 4.3$ & $6.5 \pm 4.0$ & .008 \\
\hline & Cochlear implant & $3.2 \pm 3.7$ & $3.7 \pm 3.7$ & .122 \\
\hline & Deep brain stimulation & $2.5 \pm 3.2$ & $3.8 \pm 3.7$ & $<.0005$ \\
\hline & Cortical stimulation & $2.4 \pm 3.2$ & $3.5 \pm 3.6$ & .001 \\
\hline \multirow[t]{4}{*}{$100 \%$} & Hearing aid & $5.4 \pm 4.4$ & $6.3 \pm 4.4$ & .057 \\
\hline & Cochlear implant & $3.8 \pm 3.9$ & $4.3 \pm 4.0$ & .167 \\
\hline & Deep brain stimulation & $3.0 \pm 3.6$ & $4.2 \pm 3.9$ & .001 \\
\hline & Cortical stimulation & $2.9 \pm 3.5$ & $4.1 \pm 3.8$ & .001 \\
\hline
\end{tabular}

VAS = Visual Analogue Scale

\section{Discussion}

This study assessed the willingness of patients to undergo invasive treatment for tinnitus. Around one-fifth were reasonably willing and around one-fifth were fully willing. When the chance of cure was $100 \%$ instead of $50 \%$, only a slight increase in willingness to undergo invasive treatment was found. In general, the acceptance rate was highest for $\mathrm{HA}$, followed by $\mathrm{Cl}, \mathrm{DBS}$ and $\mathrm{CS}$.

In a study by Tyler (Tyler, 2012), acceptance rates (VAS $\geq 9$ ) with a view to total relief were $38 \%, 19 \%$ and $21 \%$ for respectively $\mathrm{Cl}$, DBS and CS compared to $22 \%, 16 \%$ and $16 \%$ in our study. The lower rates in our study might be due to cultural characteristics or differences in severity. Furthermore, the population sample in the study by Tyler consisted of patients who agreed to attend a self-help group, which might have made them more willing to undergo treatment. Other dissimilarities between these two studies were the type of questionnaire (online versus paper version), the healthcare system and the number of patients ( $n=415$ versus $n=197$ ). Moreover, in Tyler's study, the questions concerned reducing tinnitus by half, whereas our questionnaire posed a $50 \%$ chance of fully eliminating it. A study on temporary implants, in particular on devices for vagal nerve stimulation, reported acceptance of $72 \%$ if the therapy would reduce annoyance by half and $91 \%$ if the tinnitus would be fully eliminated (Engineer et al., 2013). As far as we know, the willingness of patients to undergo these forms of invasive brain surgery has not been investigated for other diseases. However, it has been found that the willingness of specialists to 
refer patients for a reversible technique like DBS is almost twice as high as their propensity to refer for an irreversible lesioning technique, as demonstrated in Belgian psychiatrics for referrals among patients with Obsessive Compulsive Disorder (Gabriels et al., 2008).

The psychological distress that often accompanies tinnitus warrants attention (Grant et al., 2014). Unlike Tyler, we specified the psychological situation with the PHQ-9 and assessed the burden of tinnitus with the Mini-TQ. The average patient in our study was moderately distressed (Mini-TQ of 11) and had an indication for mild depression (PHQ-9 of 7), as found in other studies (Zoger et al., 2006, Folmer et al., 1999). Analyzing whether these and other patient characteristics were related to patient acceptance, we found weak but significant correlations with several tinnitus severity measurements and rates of treatment acceptance. The association is too weak to draw the conclusion that neuromodulatory therapies should only be offered to patients who suffer severely from tinnitus. It should be kept in mind that all participants in this study were members of a society for tinnitus patients, and therefore these results could not be extrapolated to the general tinnitus population because they might me more eager to find a treatment.

Tinnitus is a symptom of underlying pathology rather than a single disease and is most commonly associated with hearing loss. Despite limited evidence, the use of HA is usually considered for tinnitus patients who have co-existing hearing loss (Hoare et al., 2014). HA can be beneficial by compensating for the absence of auditory input (Langguth et al., 2013). Interestingly, only $52 \%$ of the patients would accept HA, even if the chance of eliminating their tinnitus is $100 \%$. Nonetheless, HA prescription is commonly the first step in tinnitus management, especially when tinnitus is accompanied by hearing loss (Hoare et al., 2012). The prevalence of hearing loss in this study was $92 \%$, suggesting a high rate of HA use (actual or attempted). HA can have a positive effect on tinnitus, in particular when the tinnitus pitch lies within the frequency of the device's amplification range (Langguth et al., 2013). Although some patients may benefit from HA, there is still insufficient evidence of its efficacy in tinnitus (Hobson et al., 2010). The most plausible reason for the low treatment acceptance of HA found in this study is prior use of HA with no positive effect on the tinnitus. Still, given a $50 \%$ chance of cure, patients with a history of tinnitus treatment would more easily accept HA (Table 3).

In general, patients who had already attempted one or more treatments were more eager to undergo an invasive therapy. This outcome measure was significant for HA, DBS and CS with a $50 \%$ chance of tinnitus elimination and for DBS and CS with a $100 \%$ chance. The attempted treatments included cognitive behavioral therapy, medication, HA and tinnitus maskers, among others. This finding supports the idea that invasive therapies should be considered as options for tinnitus patients in whom other therapies have failed.

With regard to the willingness to pay for a treatment with the prospect of complete tinnitus elimination, the option chosen most frequently (by 25\%) was paying over 20 times one's monthly income. In the Netherlands, 20 times a monthly income comes to a mean outlay of $€ 38,900$ (Statistics Netherlands, 
2014). This exceeded the amount of $\$ 5000$ found by Tyler (Tyler, 2012). At present, outlays for these invasive treatments are not reimbursed, in light of the current state of research on tinnitus.

The majority of patients would accept a risk of mild side effects and almost half of patients would accept a risk of severe side effects. Acceptance rates for deafness and death were lower; interestingly, a sizable proportion of patients would accept a $50 \%$ risk of deafness $(6 \%)$ and death (3\%). Patients who chose a higher acceptance of invasive treatments also chose higher acceptance of side effects and deafness. Risk of death was not correlated with acceptance rates. To our knowledge, no other studies have addressed willingness to accept these side effects and complications. Some caution should be taken when interpreting the acceptance rates reported here. Since these invasive procedures are often treatments of last resort, anxiety among the patients and their caregivers may lead them to underestimate the risks and rush through the informed consent protocol. In this way their consent might be based on inadequate understanding of the risks of the surgery (Grant et al., 2014). The difficulty of weighing risks and benefits could explain why the willingness to undergo invasive treatments increased only slightly when the chance of cure was $100 \%$ instead of $50 \%$. The most important ethical concerns about DBS are respecting the patient's autonomy and balancing risk vs benefit (Schermer, 2011). The present study underlines the need for comprehensive informed consent; only then would the patients and family be adequately informed about the risks, benefits and complications of the surgery (Grant et al., 2014). In this study, information on treatment consisted of a short introduction of the specific procedure followed by an overview of the potential side effects and complications (see Appendix). To prevent the potential effect of priming, the presentation was as neutral as possible. Admittedly, the risks of side effects and complications are unknown for most of the treatments, since these are not yet being performed for tinnitus. Therefore, we had to base our estimates of percentages of mild and severe side effects or complications on studies that investigated these treatments for other diseases (Kleiner-Fisman et al., 2006, Hawke et al., 1984, Venail et al., 2008, Fontaine et al., 2009). In clinical practice, patients could have more reservations about electing treatment when given a face-to-face explanation.

\section{Conclusion}

While acceptance tends to be higher for less invasive treatments, a considerable proportion of tinnitus patients would accept a variety of invasive treatments regardless of the associated risks or costs. Therefore, when preparing to conduct neuromodulatory studies for tinnitus, due attention should be given to informed consent, particularly to a thorough explanation of the potential risks.

\section{Acknowledgements}

This study was supported by the Heinsius Houbolt Foundation. We thank Marcus Janssen and Gusta van Zwieten for helpful comments on the manuscript. 


\section{References}

Arts, R. A., George, E. L., Griessner, A., Zierhofer, C. \& Stokroos, R. J. 2015. Tinnitus Suppression by Intracochlear Electrical Stimulation in Single-Sided Deafness: A Prospective Clinical Trial- Part I. Audiol Neurootol, 20, 294-313.

Baguley, D., McFerran, D. \& Hall, D. 2013. Tinnitus. Lancet, 382, 1600-7.

Chen, Y. C., Li, X., Liu, L., Wang, J., Lu, C. Q., Yang, M., Jiao, Y., Zang, F. C., Radziwon, K., Chen, G. D., Sun, W., Krishnan Muthaiah, V. P., Salvi, R. \& Teng, G. J. 2015. Tinnitus and hyperacusis involve hyperactivity and enhanced connectivity in auditory-limbic-arousal-cerebellar network. Elife, 4, e06576.

Cheung, S. W. \& Larson, P. S. 2010. Tinnitus modulation by deep brain stimulation in locus of caudate neurons (area LC). Neuroscience, $169,1768-78$

De Ridder, D., De Mulder, G., Verstraeten, E., Van der Kelen, K., Sunaert, S., Smits, M., Kovacs, S., Verlooy, J., Van de Heyning, P. \& Moller, A. R. 2006. Primary and secondary auditory cortex stimulation for intractable tinnitus. Journal for oto-rhino-laryngology and its related specialties, 68, 48-54; discussion 54-5.

Eggermont, J. J. \& Roberts, L. E. 2004. The neuroscience of tinnitus. Trends Neurosci, 27, 676-82.

Engineer, N. D., Rosellini, W. M. \& Tyler, R. S. 2013. Willingness to accept and pay for implantable tinnitus treatments: a survey. Neuromodulation, 16, 154-62.

Folmer, R. L., Griest, S. E., Meikle, M. B. \& Martin, W. H. 1999. Tinnitus severity, loudness, and depression. Otolaryngol Head Neck Surg, 121, 48-51.

Fontaine, D., Hamani, C. \& Lozano, A. 2009. Efficacy and safety of motor cortex stimulation for chronic neuropathic pain: critical review of the literature. Journal of Neurosurgy, 110, 251-6.

Gabriels, L., Nuttin, B. \& Cosyns, P. 2008. Applicants for stereotactic neurosurgery for psychiatric disorders: role of the Flemish advisory board. Acta Psychiatr Scand, 117, 381-9.

Grant, R. A., Halpern, C. H., Baltuch, G. H., O’Reardon, J. P. \& Caplan, A. 2014. Ethical considerations in deep brain stimulation for psychiatric illness. J Clin Neurosci, 21, 1-5.

Hawke, M., Wong, J. \& Krajden, S. 1984. Clinical and microbiological features of otitis externa. J Otolaryngol, 13, 289-95.

Henry, J. A., Roberts, L. E., Caspary, D. M., Theodoroff, S. M. \& Salvi, R. J. 2014. Underlying mechanisms of tinnitus: review and clinical implications. J Am Acad Audiol, 25, 5-22; quiz 126.

Hesser, H., Weise, C., Westin, V. Z. \& Andersson, G. 2011. A systematic review and meta-analysis of randomized controlled trials of cognitive-behavioral therapy for tinnitus distress. Clin Psychol Rev, 31, 545-53.

Hiller, W. \& Goebel, G. 2004. Rapid assessment of tinnitus-related psychological distress using the Mini-TQ. Int J Audiol, 43, 600-4.

Hoare, D. J., Edmondson-Jones, M., Sereda, M., Akeroyd, M. A. \& Hall, D. 2014. Amplification with hearing aids for patients with 
tinnitus and co-existing hearing loss. Cochrane Database of Systematic Reviews [Online].

Hoare, D. J., Gander, P. E., Collins, L., Smith, S. \& Hall, D. A. 2012. Management of tinnitus in English NHS audiology departments: an evaluation of current practice. J Eval Clin Pract, 18, 326-34.

Hobson, J., Chisholm, E. \& El Refaie, A. 2010. Sound therapy (masking) in the management of tinnitus in adults. Cochrane Database of Systematic Reviews, CD006371.

Jastreboff, P. J. \& Jastreboff, M. M. 2000. Tinnitus Retraining Therapy (TRT) as a method for treatment of tinnitus and hyperacusis patients. J Am Acad Audiol, 11, 162-77.

Kaltenbach, J. A. 2011. Tinnitus: Models and mechanisms. Hear Res, 276, 52-60.

Kleiner-Fisman, G., Herzog, J., Fisman, D. N., Tamma, F., Lyons, K. E., Pahwa, R., Lang, A. E. \& Deuschl, G. 2006. Subthalamic nucleus deep brain stimulation: summary and meta-analysis of outcomes. Movement Disorders, 21 Suppl 14, S290-304.

Langguth, B., Kreuzer, P. M., Kleinjung, T. \& De Ridder, D. 2013. Tinnitus: causes and clinical management. Lancet Neurology, 12, 920-30.

Luo, H., Zhang, X., Nation, J., Pace, E., Lepczyk, L. \& Zhang, J. 2012. Tinnitus suppression by electrical stimulation of the rat dorsal cochlear nucleus. Neurosci Lett.

Maes, I. H., Cima, R. F., Vlaeyen, J. W., Anteunis, L. J. \& Joore, M. A. 2013. Tinnitus: a cost study. Ear Hear, 34, 508-14.

Martinez-Devesa, P., Perera, R., Theodoulou, M. \& Waddell, A. 2010. Cognitive behavioural therapy for tinnitus. Cochrane Database Syst Rev, CD005233.

Mertens, G., De Bodt, M. \& Van de Heyning, P. 2016. Cochlear implantation as a long-term treatment for ipsilateral incapacitating tinnitus in subjects with unilateral hearing loss up to 10 years. Hear Res, 331, 1-6.

Norena, A. J. \& Farley, B. J. 2013. Tinnitus-related neural activity: theories of generation, propagation, and centralization. Hear Res, 295, 161-71.

Schermer, M. 2011. Ethical issues in deep brain stimulation. Front Integr Neurosci, 5, 17.

Shi, Y., Burchiel, K. J., Anderson, V. C. \& Martin, W. H. 2009. Deep brain stimulation effects in patients with tinnitus. Otolaryngol Head Neck Surg, 141, 285-7.

Smit, J. V., Janssen, M. L., Engelhard, M., De Bie, R. M., Schuurman, P. R., Contarino, M. F., Mosch, A., Temel, Y. \& Stokroos, R. J. $2016 a$. The impact of deep brain stimulation on tinnitus. Surgical Neurology International, 7, S848-54.

Smit, J. V., Janssen, M. L., Schulze, H., Jahanshahi, A., Van Overbeeke, J. J., Temel, Y. \& Stokroos, R. J. 2015. Deep brain stimulation in tinnitus: current and future perspectives. Brain Res, 1608, 51-65.

Smit, J. V., Janssen, M. L., van Zwieten, G., Jahanshahi, A., Temel, Y. \& Stokroos, R. J. 2016b. Deep brain stimulation of the inferior colliculus in the rodent suppresses tinnitus. Brain Res, 1650, 118-124. 
Statistics Netherlands 2014. Average income; private households related to various characteristics.

Tyler, R. S. 2012. Patient preferences and willingness to pay for tinnitus treatments. J Am Acad Audiol, 23, $115-25$.

Vanneste, S., Plazier, M., van der Loo, E., Ost, J., Meeus, O., Van de Heyning, P. \& De Ridder, D. 2011. Validation of the Mini-TQ in a Dutch-speaking population: a rapid assessment for tinnitus-related distress. B-ENT, 7, 31-6.

Venail, F., Sicard, M., Piron, J. P., Levi, A., Artieres, F., Uziel, A. \& Mondain, M. 2008. Reliability and complications of 500 consecutive cochlear implantations. Arch Otolaryngol Head Neck Surg, 134, 1276-81.

Zoger, S., Svedlund, J. \& Holgers, K. M. 2006. Relationship between tinnitus severity and psychiatric disorders. Psychosomatics, 47, 282-8.

Zuithoff, N. P., Vergouwe, Y., King, M., Nazareth, I., van Wezep, M. J., Moons, K. G. \& Geerlings, M. I. 2010. The Patient Health Questionnaire-9 for detection of major depressive disorder in primary care: consequences of current thresholds in a crosssectional study. BMC Family Practice, 11, 98. 


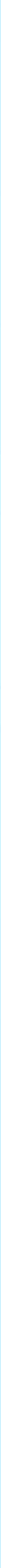




\section{Bhanter 5 \\ Deep brain stimulation of the inferior colliculus in the rodent suppresses timnitus}




\section{Abstract}

In tinnitus models pathological neuronal activity has been demonstrated. Deep brain stimulation disrupts pathological neuronal activity and might therefore be a potential treatment for patients who suffer severely from tinnitus. In this study, the effect of DBS in the inferior colliculus is investigated in an animal model for tinnitus.

The external cortex of the inferior colliculus was targeted because of the key position of the inferior colliculus within the auditory network and the relation of the external cortex with the limbic system. In this study we show the effect of DBS in the inferior colliculus on tinnitus using a within-subject experimental design.

After noise trauma, rats showed a significant increase in gap:no gap ratio of the gap-induced prepulse inhibition at 16 and $20 \mathrm{kHz}(p<0.05)$, indicating the presence of tinnitus in these frequency bands. During DBS the gap:no gap ratio returned back to baseline $(p<0.05)$. Hearing thresholds before and during DBS did not differ, indicating that hearing function is probably not impaired by electrical stimulation.

In summary, this study shows that DBS of the inferior colliculi is effective in reducing behavioral signs of tinnitus in an animal model. Impaired hearing function could not be objectified as a side effect of stimulation. 


\section{Introduction}

Tinnitus, also known as ringing of the ears, has a prevalence ranging from 10-15\% in the general population (Sanchez, 2004). While tinnitus is not bothersome in the majority of the patients, $2.4 \%$ (Axelsson and Ringdahl, 1989) of the whole population suffers daily from severely debilitating symptoms. Often tinnitus is accompanied by psychological symptoms such as anxiety and depressive symptoms (Andersson et al., 1999, Shargorodsky et al., 2010) (Milerova et al., 2013).

To date, there is consensus among the majority of researchers that noise-induced tinnitus is the result of maladaptive plasticity after damage to the peripheral auditory system (Eggermont, 2003). The decrease in auditory nerve activity leads to an increased activity in auditory as well as in non-auditory brain structures (Norena and Farley, 2013). Measurements in animal models of tinnitus show increased spontaneous neuronal firing rate, neural synchrony, bursting activity and tonotopic reorganization (Kaltenbach, 2011, Eggermont, 2003, Norena, 2011). At least some of these changes in neuronal activity have been found in the cochlear nucleus, inferior colliculus, medial geniculate body of thalamus and auditory cortex (Smit et al., 2015).

Increased bursting activity, neural synchrony and tonotopic reorganization are all demonstrated in the IC in preclinical tinnitus studies (Bauer et al., 2008, Chen and Jastreboff, 1995, Robertson et al., 2013, Wang et al., 2002). The IC integrates neuronal input from a variety of auditory nuclei. An interesting substructure within the IC is the external nucleus of the IC (ICX), which shows increased spontaneous activity and bursting activity in an animal model of tinnitus (Chen and Jastreboff, 1995) and changes in neuronal excitability following noise exposure due to neuronal plasticity (Szczepaniak and Moller, 1996). The ICX is considered part of the non-classical auditory pathway and projects via the dorsal and medial parts of the thalamic auditory nucleus to the secondary auditory cortex and auditory association cortices. Furthermore, the dorsal and medial connections from the thalamic nuclei make connections to limbic structures such as the amygdala. The limbic system reflects emotional reaction to tinnitus and might play an extended role in tinnitus (Rauschecker et al., 2010).

Currently, deep brain stimulation (DBS) is successfully applied in several central neurological conditions like Parkinson's disease and essential tremor (Larson, 2014). Via a pulse generator, electrical impulses are sent to electrodes which are surgically placed in a specific brain area with high precision. The exact mechanism of DBS is unknown but it is clear that DBS is able to modulate pathological neuronal activity patterns (McIntyre and Hahn, 2010), possibly by silencing neurons. This hypothesis is based on the fact that high-frequency stimulation results in a similar functional effect as performing a lesion of the area (Benabid et al., 2002, Hamani and Temel, 2012). The nucleus subthalamicus is a hyperactive target in Parkinson's disease and is the main target for DBS (Rodriguez-Oroz et al., 2001, Janssen et al., 2014). DBS of the ICx, which is found to be a hyperactive area in tinnitus, might reduce the tinnitus signal (Smit et al., 2015). 
The objective of this study was to assess the efficacy of DBS in the ICX in an animal model of tinnitus and to test whether DBS of the ICX induces sounds or reduces hearing.

\section{Materials and methods}

\section{Animals and experimental design}

Male Sprague Dawley rats ( $n=9,250-300$ g, Charles River, The Netherlands) were housed individually under conditions of constant temperature $\left(20-22{ }^{\circ} \mathrm{C}\right)$ and humidity $(60-70 \%)$ with a reversed light/dark cycle. Rats had ad libitum access to water and food. Experiments were ethically reviewed and approved by the Animal Experiments Committee of Maastricht University.

The study was performed using a within-subject experimental design. Tinnitus was assessed using gap induced prepulse inhibition (gPPI) of the acoustic startle reflex (Turner et al., 2006). For an overview of the measurements at different time points see Figure 1. The gPPI was first measured as a baseline measurement in naïve rats ('Baseline'). At week 6, electrodes were bilaterally implanted in the ICX. The gPPI was again measured during DBS to assess the effect of electrical stimulation on the startle response ('Baseline + DBS'). Rats were exposed to noise trauma at week 15. After noise trauma, the gPPI was randomly measured with DBS off ('Tinnitus') and DBS on ('Tinnitus + DBS'). To measure the effect of noise trauma on hearing, the auditory brainstem response (ABR) was measured before and after noise trauma to estimate hearing levels. To study the effect of potential hearing loss during stimulation, sound-induced PPI (sPPI) was measured without and with DBS at baseline. At the end of the experiment, animals were sacrificed and electrode localization was histologically confirmed.

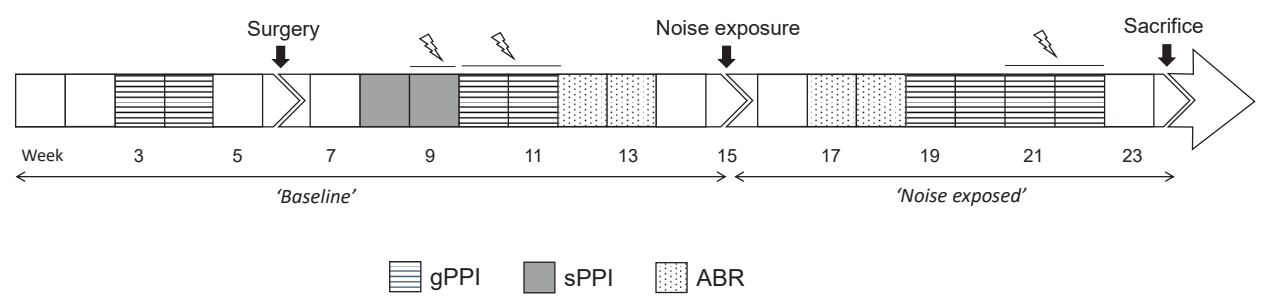

Figure 1. Schematic time line of the experiments. A lightning icon indicates that deep brain stimulation was (randomly) applied. gPPI = gap induced prepulse inhibition, SPPI = sound induced prepulse inhibition, $A B R=$ auditory brainstem response 


\section{Surgical procedure}

Induction and maintenance of the anesthesia was achieved by intraperitoneal administration of ketamine $(90 \mathrm{mg} / \mathrm{kg})$ and xylazine $(10 \mathrm{mg} / \mathrm{kg})$. The rats were fixed in a stereotactic apparatus (Stoelting Co, Wood Dale, Illinois, USA) with mouth and blunt ear bars.

Permanent Teflon-coated stainless steel wire electrodes with exposed tips were implanted for ABR measurements. The negative wire electrode was subcutaneously tunneled and placed against the mastoid bone and the positive electrode was connected to the screw on the vertex.

An extensive description of the surgical procedure for DBS has been published earlier (Tan et al. 2010). According to the stereotactic atlas (Paxinos and Watson, 2007), bilateral electrodes (Technomed, Beek, The Netherlands) were inserted in the ICX (bregma-7.9, depth 3.8, interspace 3.8). The recovery time after surgery was one week.

\section{Deep brain stimulation}

Electrical DBS stimulus pulses were created by an A310 accupulser and an A360 stimulus isolator (World Precision Instruments, Berlin, Germany). Because unilateral noise trauma can cause hyperactivity in both ICs (Ropp et al., 2014), bilateral DBS was applied. Bilateral high frequency stimulation was performed using a bipolar, concentric electrode using monophasic rectangular pulses, with a frequency of $100 \mathrm{~Hz}$, amplitude of $100 \mu \mathrm{A}$ and a pulse width of $60 \mu \mathrm{s}$. The gold plated electrodes consisted of an inner wire of platinum-iridium combination and an outer part of stainless steel, which served as the negative and positive contact, respectively. The maximum outer diameter of the electrode is approximately $250 \mu \mathrm{m}$ with a tip diameter of approximately $50 \mu \mathrm{m}$. The inner and outer electrodes are insulated except for a $75-\mu \mathrm{m}$ exposed tip. The distance between the cathode and anode is approximately $50 \mu \mathrm{m}$ (Tan et al., 2010).

Prepulse inhibition of the acoustic startle response

The presence of tinnitus was assessed with the gPPI (Turner et al., 2006). The setup was based on Tziridis et al (Tziridis et al., 2012), modified in such a way that rats were able to be connected to the stimulation cable and move freely during measurements. The setup was optimized to achieve gap:no-gap ratios of approximately 0.5 . The animals were placed in a vertical custom-made cylinder with a diameter of $17 \mathrm{~cm}$ and a height of $40 \mathrm{~cm}$. During DBS a cable was connected to the DBS sockets and a swivel was mounted on the top of the cylinder to allow rotation of the cable. The closed bottom of the cylinder was placed on a piezo sensor (FSG15N1A, Honeywell, Canada) and supported with foam plastic. The piezo sensor was connected to a 24-bit ADS1299 analogue-to-digital converter (Texas Instruments, Texas, USA) and raw data were imported in Matlab 2011a (Mathworks, Natick, Massachusetts, USA). Auditory stimuli were amplified with an Ultrasonic power amplifier and presented with an Ultrasonic Dynamic Speaker Vifa (Avisoft Bioacoustics, Berlin, Germany) that was placed above the cylinder. Sounds were 
calibrated with a Bruel \& Kjaer 2231 decibel meter with a 4191 microphone. Animals were able to freely move and were monitored with an infrared webcam.

The background sound is either a broadband noise or a narrow-banded noise of 10,12, 16 or $20 \mathrm{kHz}$ at $75 \mathrm{~dB}$. During gap trials a gap of $50 \mathrm{~ms}$ was inserted in the background sound $100 \mathrm{~ms}$ prior to the startle stimulus. The startle stimulus consisted of 20 ms click sound of $115 \mathrm{~dB}$ peak equivalent sound pressure level (peSPL). Both gap and no-gap trials were alternately executed with 20 repetitions and random variable stimulus intervals of $20 \pm 5 \mathrm{~s}$ during two separate sessions on two separate days. Rats were habituated by starting each session with 10 startle-only trails. After this period, rats lied still and were rarely moving. Trials which showed movement prior to or during the startle stimulus were discarded.

The maximum difference in deflection was defined as the startle response. The gap:no gap ratio was calculated as the startle responses from each gap-trial divided by the mean of startle-only trials. The results of two sessions were averaged.

To estimate hearing as a potential side-effect of DBS, sPPIs were measured. A similar protocol as the gPPI was used, however instead of a background noise with a gap, a prepulse sound was used (Walter et al., 2012). This sound was a broadband noise of $5 \mathrm{~ms}$ with $2 \mathrm{~ms}$ cosine-squared rise and fall ramps, ranging from 0 till $70 \mathrm{~dB}$ peSPL in steps of $10 \mathrm{~dB}$, which was $100 \mathrm{~ms}$ later followed by a 50 ms click sound of $105 \mathrm{~dB}$ peSPL that served as the startle stimulus. The mean of each trial was calculated and for each sound intensity level, the sound:no sound ratio was calculated and fitted to a Boltzmann function. Individual hearing thresholds were estimated using the 50\% function of the slope (Walter et al., 2012).

\section{Auditory brainstem response}

Ketamine $(90 \mathrm{mg} / \mathrm{kg})$ and xylazine $(10 \mathrm{mg} / \mathrm{kg})$ were intraperitoneally administered to induce anesthesia. Ketamine and xylazine have a minimal influence on the ABR (Ruebhausen et al. 2012, Smith and Mills 1989). Animals were placed into a sound-attenuating chamber which also served as a Faraday cage. ABRs were recorded at a sample frequency of $20 \mathrm{kHz}$ with PowerLab 8/35 data acquisition system connected to a Dual Bio Amp amplifier (ADInstruments, Castle Hill, Australia). During the procedure the cables from the recording device were connected to sockets on the head of the animal. The ground electrode was connected to the left hind paw.

Auditory stimuli (10, 16, 20, 24 and $32 \mathrm{kHz})$ were created with Matlab. The same calibration method and sound setup were used as for the gPPI. The stimuli were unilaterally given and consisted of $5 \mathrm{~ms}$ bursts with $\cos ^{2}$ rise and fall filter at a rate of 50 per second at decreasing intensities from 100 to $0 \mathrm{~dB}$ peSPL with steps of $10 \mathrm{~dB}$. Per frequency 1000 stimuli were given. The contralateral ear was protected from the noise with modeling clay.

Auditory stimuli were digitally triggered. The recordings were done in Labchart Pro 7 (ADInstruments, 
Castle Hill, Australia) and raw data were imported into Matlab. With a customized script, the signal was amplified 100000 times and band-pass filtered (300-3000 Hz). Evoked responses were averaged. The auditory threshold was defined as the lowest decibel level (peSPL) of the stimulus, which produced a distinctive ABR.

\section{Noise exposure}

Tinnitus was induced in all animals by a $16 \mathrm{kHz}$ octave-band noise exposure at $115 \mathrm{~dB}$ SPL for 90 minutes. The same sound system was used as for the PPI. Unilateral noise trauma was given; the contralateral ear was plugged with modeling clay. Rats were anesthetized during noise trauma with ketamine (90 $\mathrm{mg} / \mathrm{kg})$ and xylazine $(10 \mathrm{mg} / \mathrm{kg})$ for induction and ketamine $(60 \mathrm{mg} / \mathrm{kg} / \mathrm{h})$ for maintenance using an infusion pump.

\section{Histology}

At the end of the experiment animals were deeply anesthetized with pentobarbital $(75 \mathrm{mg} / \mathrm{kg}$ ) and perfused transcardially with Tyrode's buffer $(0.1 \mathrm{M})$ and fixative containing $4 \%$ paraformaldehyde, $15 \%$ picric acid and $0.05 \%$ glutaraldehyde in $0.1 \mathrm{M}$ phosphate buffer $(\mathrm{pH} 7.6)$ at $4{ }^{\circ} \mathrm{C}$. Brains were removed and post-fixed for 12 hours. Brains were serially sectioned with a vibratome.

Coronal sections containing the target area and the electrode trajectory were stained with hematoxylin-eosin (H\&E, Merck, Darmstadt, Germany) to assess the electrode localization. As a reference, the stereotactic atlas was used (Paxinos and Watson, 2007).

\section{Statistics}

Tinnitus was assessed as a statistical significant grand mean group difference between the situation after noise trauma compared to baseline. Statistical analyses for PPI and ABR data were performed using the Wilcoxon signed-rank test to compare within-subject group differences. $\mathrm{P}<0.05$ was considered significantly different. Data were analyzed by using SPSS (Version 20, IBM, Somers, New York, USA). 


\section{Results}

\section{Electrode localization}

The electrode trajectories were reviewed on slides after H\&E staining. In all animals, the electrode tips were correctly placed within the ICx (see Figure 2). We observed no additional histological damage due to DBS.

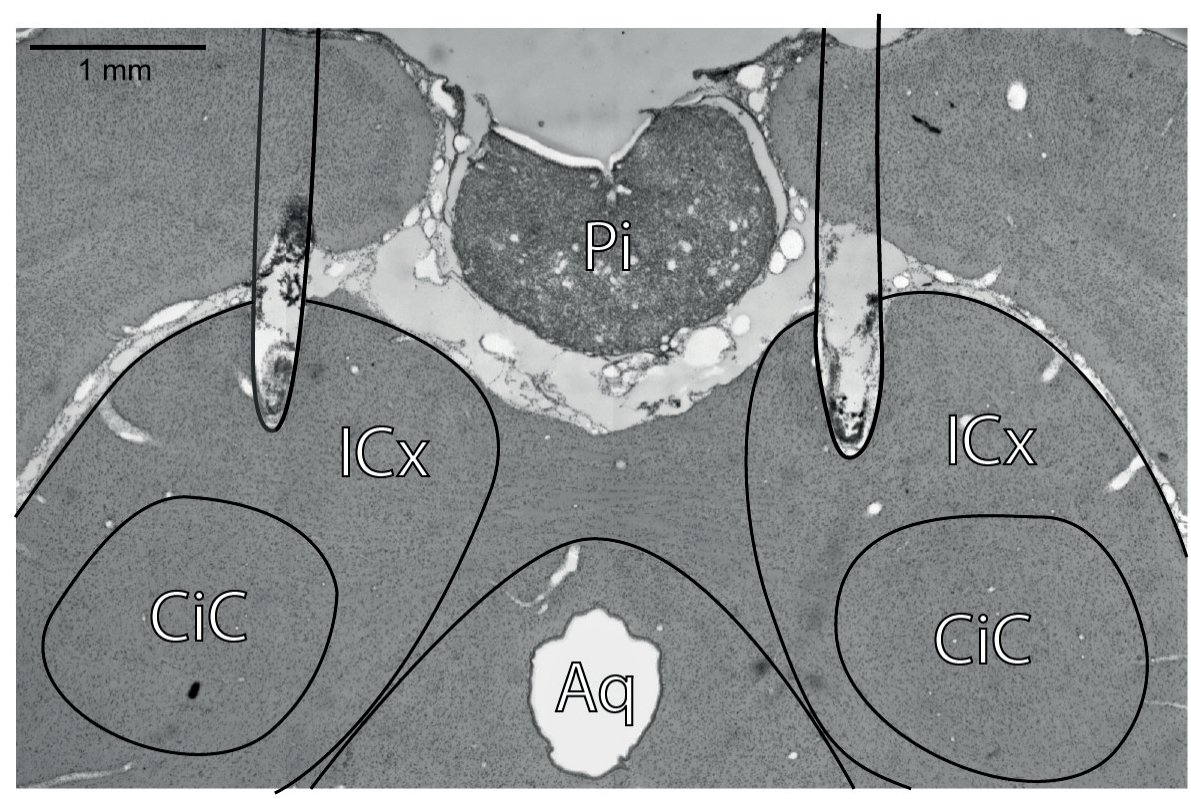

Figure 2. H\&E staining showing a representative example of the bilateral electrode trajectories. The electrode tip is situated within the ICX. ICX = external cortex of inferior colliculus, CiC=central nucleus of inferior colliculus, Aq=aquaduct, $P i=$ pineal gland

\section{Hearing thresholds after noise trauma}

In Figure 3 the hearing thresholds according to auditory brainstem responses are illustrated at baseline and after noise trauma. Hearing thresholds significantly increased measured the ipsilateral ear after noise trauma at all frequencies $(p<0.05)$, while no differences were seen in the contralateral ear. In one rat $A B R$ measurements were technically not possible after noise trauma due to an electrode defect and this rat was therefore excluded for assessing hearing thresholds. 


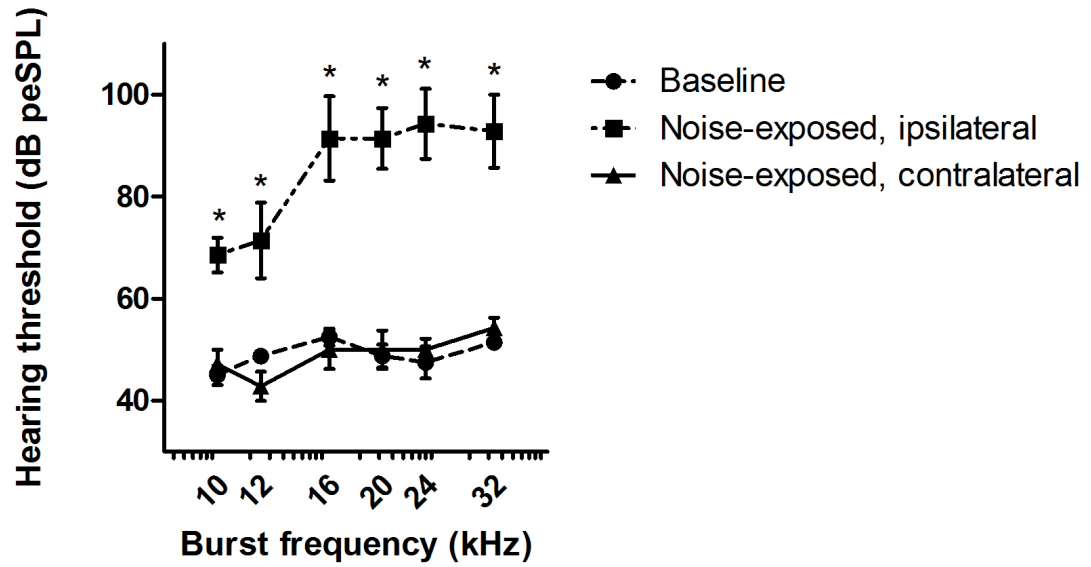

Figure 3. Auditory brainstem responses (ABRS) measured at baseline (round, solid), during tinnitus measured at the ipsilateral ear (squares, dotted) and during tinnitus measured at the contralateral ear (triangle, solid). The $x$-axis is the frequency of the sound on a logarithmic scale. An asterisk indicates a significant difference compared to baseline $(p<0.05)$.

\section{Tinnitus assessment}

Following noise trauma, gap:no-gap ratios increased significantly in the 16 and $20 \mathrm{kHz}$ banded background sound $(p<0.05)$. During DBS treatment, gap:no-gap ratios returned to baseline after noise trauma (see Figure 4). The gap:no-gap ratios did not change significantly during DBS at baseline compared to baseline without stimulation. 
A
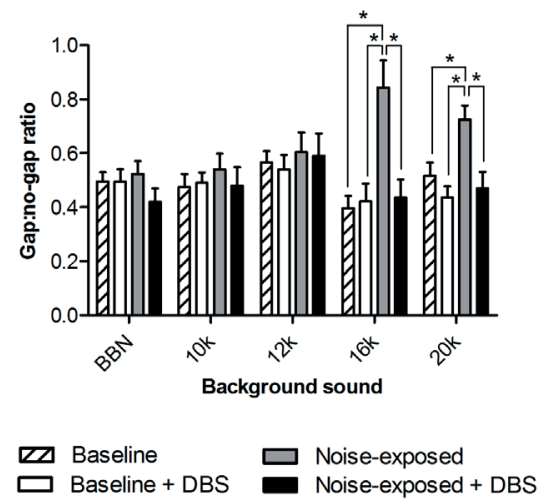

10k

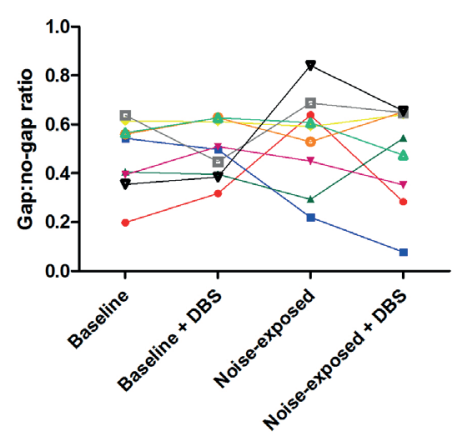

$16 k$

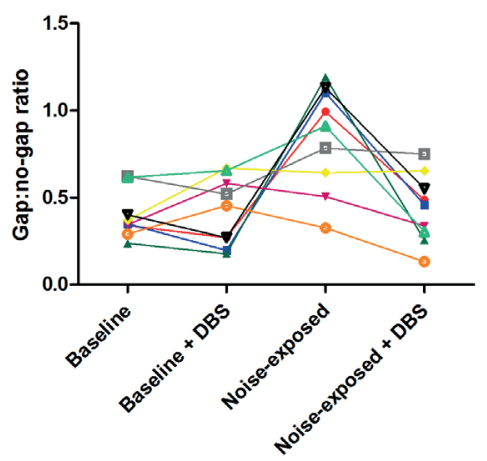

B

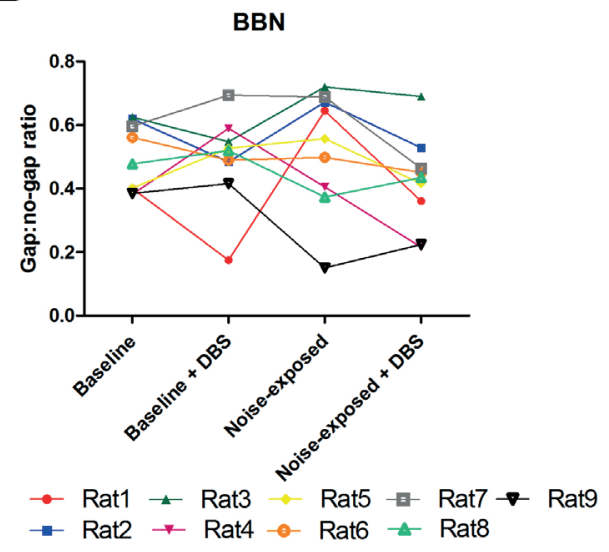

12k

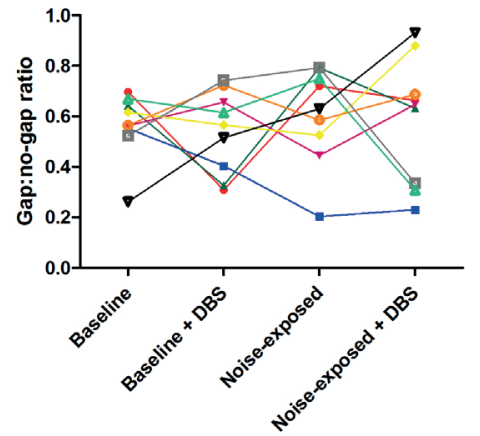

20k

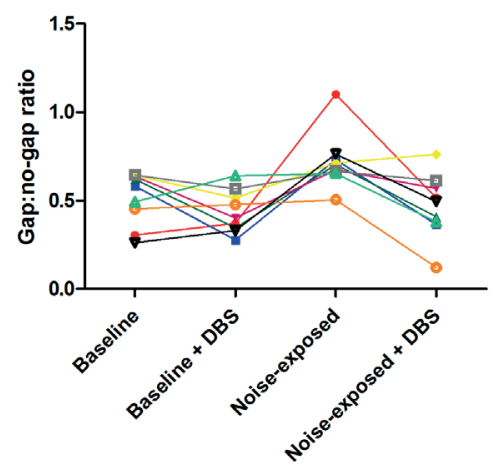

Figure 4. Grand mean (A) and individual data (B) of the gap induced prepulse inhibition ( $g$ PPI) of the acoustic startle reflex at baseline (naive animals), and during tinnitus, without and with DBS. An asterisk indicates a significant difference $(p<0.05)$. BBN $=b r o a d b a n d$ noise, the rest are background sounds expressed in $\mathrm{Hz}$. 


\section{Hearing during ICX DBS}

The sPPI was measured during the baseline situation without and with DBS. The results were individually fitted to a Boltzman function and the 50\% function of the slope was derived (for an example see Figure $5 a)$. There was no statistical significant difference between on and off stimulation ( $p>0.05)$. The $50 \%$ function of the slope of the Boltzman function of the grand mean for the DBS off state was 39.8 and for the DBS on state 37.3. For the fitting of the grand mean of the sPPI see Figure $5 b$.
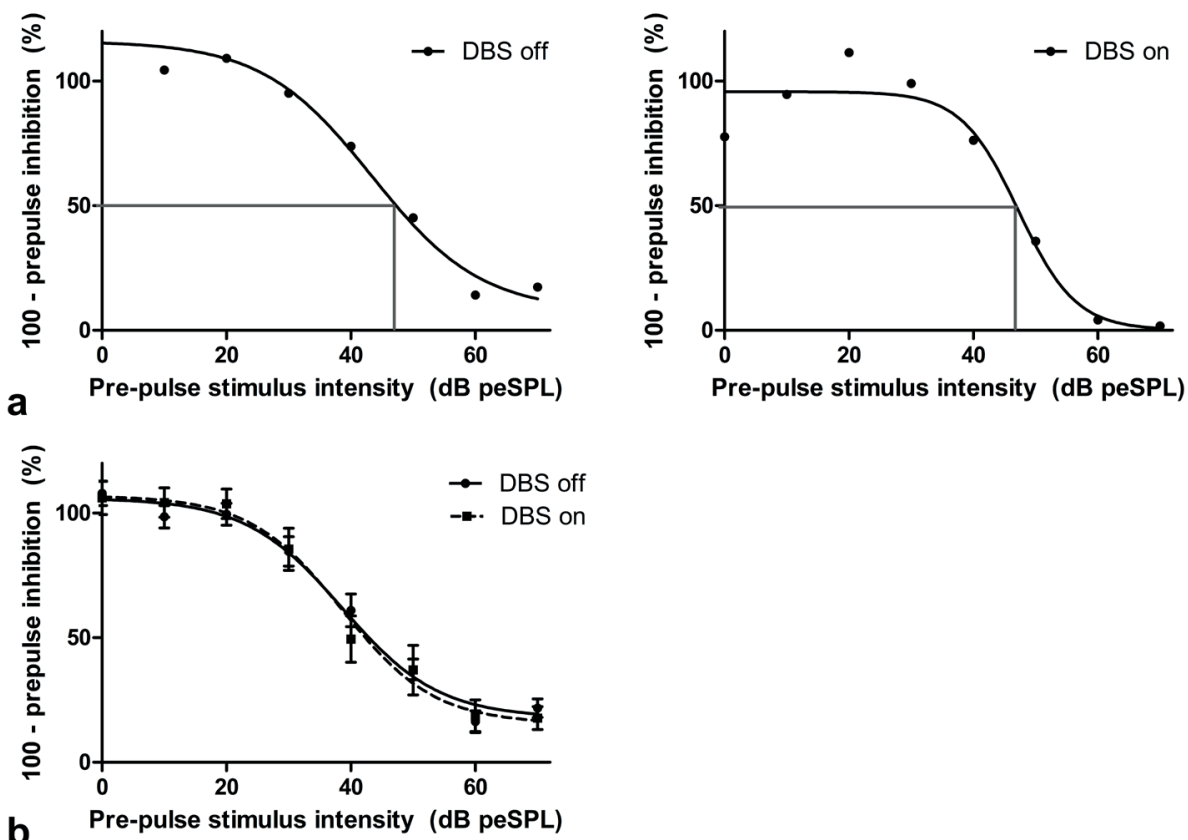

Figure 5. During baseline situation, sound induced prepulse inhibition (sPPI) was assessed with and without deep brain stimulation (DBS). a. Two representative examples of sPPIs with Boltzman functions. The gray lines indicate how the 50\% function of the slope is calculated, $b$. Grand mean of the sound:no sound ratio measured with the SPPI. 


\section{Discussion}

As far as we are aware of, this is the first study which provided evidence that ICX DBS reduces tinnitus in a validated animal model. This is proven by a decrease in the gap:no-gap ratio during DBS, which is increased after noise trauma. We provided indirect evidence by the SPPI that DBS does not impair hearing.

Measurements of the gPPI of the acoustic startle response is nowadays the most common approach to assess tinnitus in animal models (Galazyuk and Hebert, 2015). With this method tinnitus can be measured repeatedly, rapidly and conditioning paradigms are not required. Several methodological aspects have to be considered for reliable measurements (Turner et al., 2006, Lobarinas et al., 2012, Longenecker and Galazyuk, 2012). In order to make it possible to connect the animals to the stimulation cable, the startle chamber had to be adjusted in a way that the animals could freely move. Previous studies have also used the gPPI during electrical stimulation (Engineer et al., 2011, Luo et al., 2012). Stress is minimized by testing the animals while they could freely move which approaches the natural startle condition best. To correct for the increased variability that occurred as a consequence of the fact that the animals could freely move, grand means of gap:no-gap ratios of all rats were used to statistically analyze tinnitus-like behavior. To minimize confounders in the assessment of tinnitus, we applied unilateral noise trauma and verified with ABR measurements that the hearing thresholds did not alter in the contralateral ear. These results indicate that animals could still hear the background and stimulus sounds after noise trauma. Furthermore, by using the gap:no-gap ratios instead of absolute measurements of amplitudes of startle responses, an increase in ratio is less likely to be explained by hearing loss. Hearing thresholds were increased in all tested frequencies (Figure 3), but tinnitus was only seen in 16 and $20 \mathrm{kHz}$ frequency bands (Figure 4). One would expect that if the increased gap:no-gap ratios would only be explained by hearing loss that also other frequency bands would show the same increase as seen in the 16 and $20 \mathrm{kHz}$ bands. Hyperacusis is another potential confounder in the startle paradigm. It has been found that a $115 \mathrm{~dB}$ noise trauma of one hour can result in masking of tinnitus while a $110 \mathrm{~dB}$ noise trauma of two hours can result in measurements of pseudo-tinnitus (Salloum et al., 2016). It is possible that hyperacusis plays a confounding role in this study. Furthermore, based on previous studies, it is likely that some animals did not develop tinnitus after noise exposure (Engineer et al., 2011, Turner and Larsen, 2016). The found results can therefore be an underestimation of the real effect.

In animals, tinnitus can be induced by salicylates or noise trauma. While salicylates produce a reversible and temporary form of tinnitus, noise trauma results in chronic tinnitus and represents the cause of tinnitus in humans best (Brozoski and Bauer, 2015). For this reason, we chose noise trauma to induce tinnitus. Tinnitus was measured in 16 and $20 \mathrm{kHz}$ frequency bands. It is likely that the generated tinnitus is a tonal sound with a pitch around 16 and $20 \mathrm{kHz}$. It is common to experience tinnitus in the frequency region of hearing loss (Eggermont and Roberts, 2015). In a recent study that compared the effect of several noise trauma paradigms on tinnitus, a $16 \mathrm{kHz}$ octave band sound trauma was found to lead to 
tinnitus measured in the $16 \mathrm{kHz}$ frequency band, which is similar to our study, although we also found tinnitus in the $20 \mathrm{kHz}$ frequency band. Tinnitus was more often found after a $16 \mathrm{kHz} 110 \mathrm{~dB}$ noise trauma than a $16 \mathrm{kHz} 116 \mathrm{~dB}$ sound (Turner and Larsen, 2016). A direct comparison on sound intensity with our study cannot be made since we used a different method for sound calibration.

The most potential side-effect when electrically stimulating the IC is hearing loss. To estimate hearing loss without and with DBS we measured the sPPI. Estimation of hearing thresholds using the SPPI is based on the ability to hear sounds which inhibit the startle caused by a startle stimulus (Walter et al., 2012). Based on individual statistical analysis, no statistical significant difference was found. When comparing the Boltzman functions of the grand means, the function of the DBS state was slightly shifted to the left. A decrease in hearing would have resulted in a shift of the function to the right. The sPPI was measured with clicks, and thus is not frequency specific, and represents an overall estimation of hearing function.

DBS in naive rats did not show any effects on the gap:no-gap ratios. It has been shown that large lesions of the IC eliminate the inhibition of the acoustic startle by an auditory prestimulus (Leitner and Cohen, 1985, Li et al., 1998). In theory, if due to DBS the whole IC would be 'silenced', a high gap:no-gap ratio would be found, masking the effect of treatment. With the current experimental setup, no effect of stimulation of the ICX on the PPI was seen in naive rats. Therefore, it is likely that DBS in the ICX does not affect the neuronal network loop that is related to the mechanism of PPI. Furthermore, acoustic sounds can mask electrical stimuli in auditory centers such as the dorsal cochlear nucleus but not in nearby non-auditory neuronal structures (Frederickson and Gerken, 1978). The high-frequency DBS pulses are likely not or at least not negatively influenced by the auditory stimuli, since there was a clear effect of DBS on gap:no-gap ratios of the tinnitus-related frequencies (16 kHz and $20 \mathrm{kHz}$ background sound).

Several forms of neural stimulation strategies for tinnitus have been proposed and tested. Non-invasive stimulation has great potential because of the general applicability. However, most recent randomized clinical trials fail to show a beneficial effect (Langguth et al., 2014, Pal et al., 2015, Hoekstra et al., 2013), possibly due to minimal invasion depth in the brain. Invasive electrical stimulation showed moderate effects when stimulating the eight cranial nerve (Holm et al., 2005) or auditory cortex (De Ridder et al., 2011). Cochlear stimulation is a promising treatment for tinnitus, but is only feasible in patients with uni- or bilateral deafness due to the high risk of surgical iatrogenic cochlear damage (Arts et al., 2012, Ramakers et al., 2015). Cochlear stimulation seems to be able to influence tonotopic reorganization by activating auditory nerve fibers (Di Nardo et al., 2007). Therefore, DBS might be a promising treatment for severe, refractory tinnitus sufferers with residual hearing.

Several auditory as well as non-auditory brain structures are known to be involved in tinnitus (Smit et al., 2015). Subcortical auditory structures that showed altered neuronal activity are the cochlear nucleus, IC and medial geniculate body of the thalamus. Auditory structures are potential structures for selective tinnitus treatment since it is likely that these structures play a key role in the pathological 
neuronal network. To our knowledge no other studies are published which investigated the effect of ICX DBS on tinnitus. In guinea pigs, low frequency stimulation has been applied in the dorsal cochlear nucleus. During stimulation the gap:no-gap ratio significantly decreased. Interestingly, the effect persisted after turning off DBS (Luo et al., 2012). This could be a result of residual inhibition which is also seen in patients treated with thalamic DBS (Shi et al., 2009). The setup of the study of Luo and colleagues is not directly comparable to our study and therefore, no conclusions can be made regarding target preferences for future clinical DBS implementation.

The central nucleus of the IC already has been stimulated for hearing restoration in patients with neurofibromatosis type II (Offutt et al., 2014). In this study, electrodes are implanted via a craniotomy. A stereotactical approach is less invasive than a craniotomy and is currently widely applied in DBS in for example the subthalamic nucleus of the thalamus. If intervention anywhere in the pathological tinnitus pathway is sufficient for tinnitus reduction, alternative structures like the medical geniculate body of the thalamus which are better accessible with stereotaxy need to be studied. Animal studies are desirable to explore the feasibility of the various potential targets.

The stimulation parameters used in the current study are widely used in animal models for Parkinson's disease, and stimulation with these parameters reduced motor symptoms (Temel et al., 2005). Obviously, the most optimal parameters for stimulation for tinnitus have yet to be determined and, similar as clinical DBS, might need to be individually adjusted.

\section{Conclusions}

In conclusion, we have found that DBS of the ICX is effective in reducing behavioral evidence of tinnitus in the 16 and $20 \mathrm{kHz}$ frequency bands. Hearing seems not to be affected by DBS. Using translational animal models systematically, novel therapeutic strategies might be established for the treatment of tinnitus. 


\section{References}

Andersson, G., Lyttkens, L. \& Larsen, H. C. 1999. Distinguishing levels of tinnitus distress. Clin Otolaryngol Allied Sci, 24, 404-10.

Arts, R. A., George, E. L., Stokroos, R. J. \& Vermeire, K. 2012. Review: cochlear implants as a treatment of tinnitus in single-sided deafness. Curr Opin Otolaryngol Head Neck Surg, 20, 398-403.

Axelsson, A. \& Ringdahl, A. 1989. Tinnitus--a study of its prevalence and characteristics. Br J Audiol, $23,53-62$.

Bauer, C. A., Turner, J. G., Caspary, D. M., Myers, K. S. \& Brozoski, T. J. 2008. Tinnitus and inferior colliculus activity in chinchillas related to three distinct patterns of cochlear trauma. J Neurosci Res, 86, 2564-78.

Benabid, A. L., Benazzous, A. \& Pollak, P. 2002. Mechanisms of deep brain stimulation. Mov Disord, 17 Suppl 3, S73-4.

Brozoski, T. J. \& Bauer, C. A. 2015. Animal models of tinnitus. Hear Res.

Chen, G. D. \& Jastreboff, P. J. 1995. Salicylate-induced abnormal activity in the inferior colliculus of rats. Hear Res, 82, 158-78.

De Ridder, D., Vanneste, S., Kovacs, S., Sunaert, S., Menovsky, T., van de Heyning, P. \& Moller, A. 2011. Transcranial magnetic stimulation and extradural electrodes implanted on secondary auditory cortex for tinnitus suppression. J Neurosurg, $114,903-11$.

Di Nardo, W., Cantore, I., Cianfrone, F., Melillo, P., Scorpecci, A. \& Paludetti, G. 2007. Tinnitus modifications after cochlear implantation. Eur Arch Otorhinolaryngol, 264, 1145-9.

Eggermont, J. J. 2003. Central tinnitus. Auris Nasus Larynx, 30 Suppl, S7-12.

Eggermont, J. J. \& Roberts, L. E. 2015. Tinnitus: animal models and findings in humans. Cell Tissue Res, 361, $311-36$.

Engineer, N. D., Riley, J. R., Seale, J. D., Vrana, W. A., Shetake, J. A., Sudanagunta, S. P., Borland, M. S. \& Kilgard, M. P. 2011. Reversing pathological neural activity using targeted plasticity. Nature, 470, 101-4.

Frederickson, C. J. \& Gerken, G. M. 1978. Functional characteristics of cochlear nucleus in behaving cat examined by acoustic masking of electrical stimuli. J Neurophysiol, 41, 1535-45.

Galazyuk, A. \& Hebert, S. 2015. Gap-Prepulse Inhibition of the Acoustic Startle Reflex (GPIAS) for Tinnitus Assessment: Current Status and Future Directions. Front Neurol, 6, 88.

Hamani, C. \& Temel, Y. 2012. Deep brain stimulation for psychiatric disease: contributions and validity of animal models. Sci Trans/ Med, 4, 142 rv8.

Hoekstra, C. E., Versnel, H., Neggers, S. F., Niesten, M. E. \& van Zanten, G. A. 2013. Bilateral low-frequency repetitive transcranial magnetic stimulation of the auditory cortex in tinnitus patients is not effective: a randomised controlled trial. Audiol Neurootol, $18,362-73$.

Holm, A. F., Staal, M. J., Mooij, J. J. \& Albers, F. W. 2005. Neurostimulation as a new treatment for severe tinnitus: a pilot study. Otol Neurotol, 26, 425-8; discussion 428. 
Janssen, M. L., Duits, A. A., Turaihi, A. M., Ackermans, L., Leentjens, A. F., van Kranen-Mastenbroek, V., Oosterloo, M., Visser-Vandewalle, V. \& Temel, Y. 2014. Subthalamic nucleus high-frequency stimulation for advanced Parkinson's disease: motor and neuropsychological outcome after 10 years. Stereotact Funct Neurosurg, 92, 381-7.

Kaltenbach, J. A. 2011. Tinnitus: Models and mechanisms. Hear Res, 276, 52-60.

Langguth, B., Landgrebe, M., Frank, E., Schecklmann, M., Sand, P. G., Vielsmeier, V., Hajak, G. \& Kleinjung, T. 2014. Efficacy of different protocols of transcranial magnetic stimulation for the treatment of tinnitus: Pooled analysis of two randomized controlled studies. World J Biol Psychiatry, 15, 276-85.

Larson, P. S. 2014. Deep brain stimulation for movement disorders. Neurotherapeutics, 11, 465-74.

Leitner, D. S. \& Cohen, M. E. 1985. Role of the inferior colliculus in the inhibition of acoustic startle in the rat. Physiol Behav, 34, 65-70.

Li, L., Priebe, R. P. \& Yeomans, J. S. 1998. Prepulse inhibition of acoustic or trigeminal startle of rats by unilateral electrical stimulation of the inferior colliculus. Behav Neurosci, 112, 1187-98.

Lobarinas, E., Hayes, S. H. \& Allman, B. L. 2012. The gap-startle paradigm for tinnitus screening in animal models: Limitations and optimization. Hear Res, 296, 150-160.

Longenecker, R. J. \& Galazyuk, A. V. 2012. Methodological optimization of tinnitus assessment using prepulse inhibition of the acoustic startle reflex. Brain Res.

Luo, H., Zhang, X., Nation, J., Pace, E., Lepczyk, L. \& Zhang, J. 2012. Tinnitus suppression by electrical stimulation of the rat dorsal cochlear nucleus. Neurosci Lett, 522, 16-20.

McIntyre, C. C. \& Hahn, P. J. 2010. Network perspectives on the mechanisms of deep brain stimulation. Neurobiol Dis, 38, 329-37.

Milerova, J., Anders, M., Dvorak, T., Sand, P. G., Koniger, S. \& Langguth, B. 2013. The influence of psychological factors on tinnitus severity. Gen Hosp Psychiatry, 35, 412-6.

Norena, A. J. 2011. An integrative model of tinnitus based on a central gain controlling neural sensitivity. Neuroscience and biobehavioral reviews, 35, 1089-109.

Norena, A. J. \& Farley, B. J. 2013. Tinnitus-related neural activity: theories of generation, propagation, and centralization. Hear Res, $295,161-71$.

Offutt, S. J., Ryan, K. J., Konop, A. E. \& Lim, H. H. 2014. Suppression and facilitation of auditory neurons through coordinated acoustic and midbrain stimulation: investigating a deep brain stimulator for tinnitus. J Neural Eng, 11, 066001.

Pal, N., Maire, R., Stephan, M. A., Herrmann, F. R. \& Benninger, D. H. 2015. Transcranial Direct Current Stimulation for the Treatment of Chronic Tinnitus: A Randomized Controlled Study. Brain Stimul, 8, 1101-1107.

Paxinos, G. \& Watson, C. 2007. The Rat Brain in Stereotaxic Coordinates, London, Academic Press.

Ramakers, G. G., van Zon, A., Stegeman, I. \& Grolman, W. 2015. The effect of cochlear implantation on tinnitus in patients with bilateral hearing loss: A systematic review. Laryngoscope, 125, 2584-92. 
Rauschecker, J. P., Leaver, A. M. \& Muhlau, M. 2010. Tuning out the noise: limbic-auditory interactions in tinnitus. Neuron, 66, 819-26.

Robertson, D., Bester, C., Vogler, D. \& Mulders, W. H. 2013. Spontaneous hyperactivity in the auditory midbrain: relationship to afferent input. Hear Res, 295, 124-9.

Rodriguez-Oroz, M. C., Rodriguez, M., Guridi, J., Mewes, K., Chockkman, V., Vitek, J., DeLong, M. R. \& Obeso, J. A. 2001. The subthalamic nucleus in Parkinson's disease: somatotopic organization and physiological characteristics. Brain, 124, 1777-90.

Ropp, T. J., Tiedemann, K. L., Young, E. D. \& May, B. J. 2014. Effects of unilateral acoustic trauma on tinnitus-related spontaneous activity in the inferior colliculus. J Assoc Res Otolaryngol, 15, 1007-22.

Salloum, R. H., Sandridge, S., Patton, D. J., Stillitano, G., Dawson, G., Niforatos, J., Santiago, L. \& Kaltenbach, J. A. 2016. Untangling the effects of tinnitus and hypersensitivity to sound (hyperacusis) in the gap detection test. Hear Res, 331, 92-100.

Sanchez, L. 2004. The Epidemiology of Tinnitus. Audiological Medicine, 2, 8-17.

Shargorodsky, J., Curhan, G. C. \& Farwell, W. R. 2010. Prevalence and characteristics of tinnitus among US adults. Am J Med, 123, 711-8.

Shi, Y., Burchiel, K. J., Anderson, V. C. \& Martin, W. H. 2009. Deep brain stimulation effects in patients with tinnitus. Otolaryngol Head Neck Surg, 141, 285-7.

Smit, J. V., Janssen, M. L., Schulze, H., Jahanshahi, A., Van Overbeeke, J. J., Temel, Y. \& Stokroos, R. J. 2015. Deep brain stimulation in tinnitus: current and future perspectives. Brain Res, 1608, 51-65.

Szczepaniak, W. S. \& Moller, A. R. 1996. Evidence of neuronal plasticity within the inferior colliculus after noise exposure: a study of evoked potentials in the rat. Electroencephalogr Clin Neurophysiol, 100, 158-64.

Temel, Y., Visser-Vandewalle, V., Aendekerk, B., Rutten, B., Tan, S., Scholtissen, B., Schmitz, C., Blokland, A. \& Steinbusch, H. W. 2005. Acute and separate modulation of motor and cognitive performance in parkinsonian rats by bilateral stimulation of the subthalamic nucleus. Exp Neurol, 193, 43-52.

Turner, J. G., Brozoski, T. J., Bauer, C. A., Parrish, J. L., Myers, K., Hughes, L. F. \& Caspary, D. M. 2006. Gap detection deficits in rats with tinnitus: a potential novel screening tool. Behav Neurosci, 120, 188-95.

Turner, J. G. \& Larsen, D. 2016. Effects of noise exposure on development of tinnitus and hyperacusis: Prevalence rates 12 months after exposure in middle-aged rats. Hear Res, 334, 30-6.

Tziridis, K., Ahlf, S. \& Schulze, H. 2012. A low cost setup for behavioral audiometry in rodents. J Vis Exp.

Walter, M., Tziridis, K., Ahlf, S. \& Schulze, H. 2012. Context Dependent Auditory Thresholds Determined by Brainstem Audiometry and Prepulse Inhibition in Mongolian Gerbils. Open Journal of Acoustics, 2, 34-49.

Wang, J., Ding, D. \& Salvi, R. J. 2002. Functional reorganization in chinchilla inferior colliculus associated with chronic and acute cochlear damage. Hear Res, 168, 238-49. 


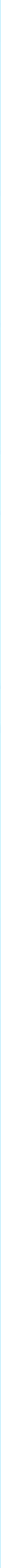




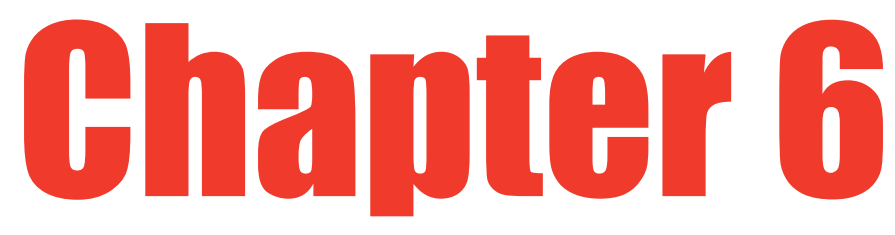

\section{Hearing assessment during deep brain stimulation of the central nucleus of the inferior colliculus and dentate cerebellar nucleus in rat}

JV Smit, A Jahanshahi, MLF Janssen, RJ Stokroos, Y Temel PeerJ (2017) 5:e3892 


\section{Abstract}

Recently it has been shown in animal studies that deep brain stimulation (DBS) of auditory structures was able to reduce tinnitus-like behavior. However, the question arises whether hearing might be impaired when interfering in auditory-related network loops with DBS.

The auditory brainstem response (ABR) was measured in rats during high frequency stimulation (HFS) and low frequency stimulation (LFS) in the central nucleus of the inferior colliculus ( $C I C, n=5)$ or dentate cerebellar nucleus ( $D C B N, n=5$ ). Besides hearing thresholds using $A B R$, relative measures of latency and amplitude can be extracted from the ABR. In this study ABR thresholds, interpeak latencies (I-III, III-V, $\mathrm{I}-\mathrm{V})$ and $\mathrm{V} / \mathrm{I}$ amplitude ratio were measured during off-stimulation state and during LFS and HFS.

In both the CIC and the CNBN groups, no significant differences were observed for all outcome measures.

DBS in both the CIC and the CNBN did not have adverse effects on hearing measurements. These findings suggest that DBS does not hamper physiological processing in the auditory circuitry. 


\section{Introduction}

Deep brain stimulation (DBS) in auditory structures has been performed in animal studies as a treatment for tinnitus (Luo et al., 2012, Smit et al., 2016). The rationale behind this treatment is to interfere with the pathological neuronal activity in the central nervous system and interrupt the network loop that is essential for the persistence of tinnitus (Smit et al., 2015).

The fundamental knowledge of the effect of deep DBS in auditory structures on hearing is essential before applying this treatment in a clinical setting (Smit et al., 2015). It has been shown in rats, using the sound-induced pre-pulse inhibition test with click stimuli, that during high frequency stimulation (HFS) of the external nucleus of the informed colliculus (IC) hearing thresholds did not change (Smit et al., 2016). As far as we know, a more detailed hearing assessment during DBS in auditory structures has not been assessed thus far.

To assess hearing thresholds in more detail, the auditory brainstem response (ABR) was measured in this study. The ABR assesses changes in neural integrity and is commonly used in laboratory animal studies to estimate hearing (Rosahl et al., 2000, Turner et al., 2006). In humans, ABRs are used in daily practice to assess possible hearing loss of a retrocochlear origin (Stockard and Rossiter, 1977).

Two structures were targeted in this study, the central nucleus of the IC $(\mathrm{CIC})$ and the dentate cerebellar nucleus (DCBN). The CIC is the principal auditory part of the IC and has a well-defined tonotopy (Aitkin and Moore, 1975, De Martino et al., 2013). In animal models of tinnitus, the IC shows tonotopic reorganization, increased spontaneous firing rate, increased bursting activity and increased neural synchrony (Bauer et al., 2008, Robertson et al., 2013, Wang et al., 2002, Chen and Jastreboff, 1995). A recent study showed that HFS of the external nucleus of the inferior colliculus (IC) in rats decreased tinnitus-like behavior (Smit et al., 2016). The cerebellum is a structure that is not involved in the auditory pathways but is associated with tinnitus (Brozoski et al., 2007, Sedley et al., 2012, Osaki et al., 2005, Shulman and Strashun, 1999). It was demonstrated that ablation of the paraflocculus completely diminished tinnitus in rats (Bauer et al., 2012). The majority of fibers in the cerebellum, including the paraflocculus, originate from the deep cerebellar nuclei, especially the DCBN, which is the largest (Gayer and Faull, 1988, Gould, 1979). Therefore, the CIC and the DCBN could be considered as respectively an auditory and a non-auditory potential DBS target for the treatment of tinnitus.

DBS can be performed with low frequency stimulation (LFS), which mainly has an excitatory effect, and as HFS, which generally is described as a global inhibitory effect similar as ablation (Benabid et al., 1998, Breit et al., 2004, Dostrovsky and Lozano, 2002). Following ablation of IC in animals models, decreased amplitude and latency of peak V have been found (Achor and Starr, 1980, Buchwald and Huang, 1975, Durrant et al., 1994, Kaga et al., 1997). Peak $V$ is the last of the five peaks of the ABR and represents neural activity of the IC. Because of a high variability in amplitude among subjects, the $\mathrm{V} / \mathrm{I}$ amplitude ratio is a more consistent measure than the absolute value (Musiek et al., 1984, Musiek et al., 1985). 
The relative measures of the latencies are the interpeak latencies (I-III, III-V, I-V) which represent the central transmission latency best (Eggermont and Don, 1986, Picton et al., 1977, Squires et al., 1978). There is little evidence that stimulation of cerebellar structures has influence on the ABR (Crispino and Bullock, 1984).

We hypothesize that for $\mathrm{CIC}$ stimulation, the $\mathrm{V} / \mathrm{I}$ amplitude ratio of the ABR would be lower and the I-V or III-V interpeak latencies would be prolonged during HFS and not during LFS of the CIC. Our hypothesis is that stimulating a non-auditory structure such as the DCBN does not have any influence on the ABR.

\section{Methods}

\section{Animals}

Male rats (Sprague Dawley, 250-300 g, Charles River, The Netherlands) were housed individually under conditions of constant room temperature and humidity with a reversed $12 \mathrm{u} / 12 \mathrm{u}$ light/dark cycle and had free access to water and food. The Animal Experiments Committee of the Maastricht University approved the experiments (approval reference number 2012-069).

\section{Surgical procedure}

Subcutaneous electrodes were implanted for ABR recordings and during the same surgery DBS electrodes were implanted in the brain (Figure 1). Animals were anesthetized by intraperitoneal administration of ketamine $(90 \mathrm{mg} / \mathrm{kg})$ and xylazine $(10 \mathrm{mg} / \mathrm{kg})$. The head of the rats was immobilized in a stereotactic apparatus (Stoelting Co, Wood Dale, Illinois) with mouth and blunt ear-bars. Permanent Teflon-coated stainless steel electrodes were subcutaneously implanted. One wire electrode was subcutaneously tunneled to the mastoid and a second wire electrode was attached to a screw on the vertex. Based on coordinates from a stereotactic atlas (Paxinos and Watson, 2007), bilateral electrodes (Technomed, Beek, The Netherlands) were inserted in the CIC (bregma-8.8, depth 4.5, interspace 3.8) or in the DCBN (bregma-11.5, depth 6.5, interspace 6.8). The postoperative recovery time was one week. 


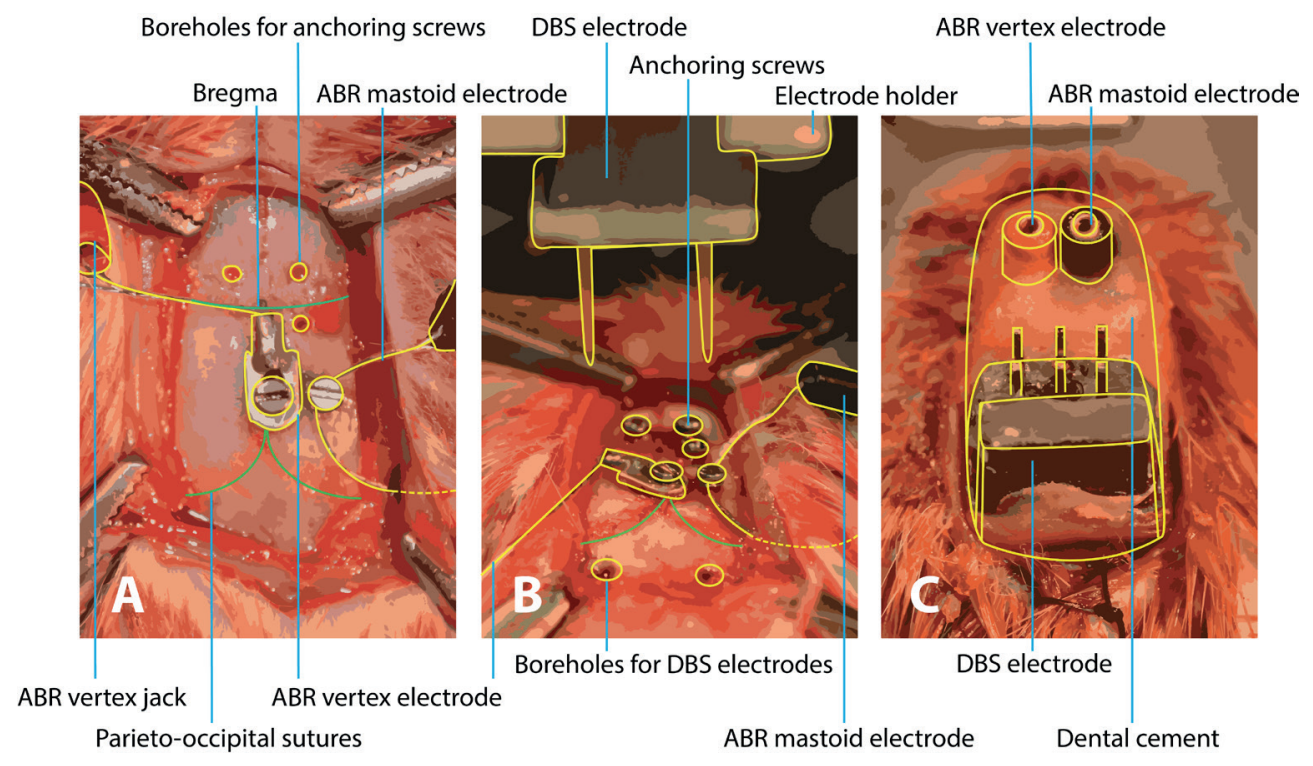

Figure 1. A. After exposing the skull, the vertex electrode is attached with a screw in the skull and the mastoid electrode is subcutaneously tunneled to the mastoid and also fixated with a screw. Three boreholes are made for anchoring screws to later fixate the structure with dental cement. B. Boreholes for the DBS electrodes are drilled at coordinates calculated from the bregma level. Calculation of the boreholes and placement of the DBS electrodes are performed within a stereotactic frame. C. All electrodes are in place and the construct is fixated with dental cement.

\section{Deep brain stimulation}

DBS was performed with bipolar, concentric electrodes using monophasic rectangular pulses. The electrical stimulus pulses were created by an A310 accupulser and an A360 stimulus isolator (World Precision Instruments, Berlin, Germany). During DBS, stimuli were given with a frequency of $100 \mathrm{~Hz}$ (HFS) and $10 \mathrm{~Hz}$ (LFS) with an amplitude of $100 \mathrm{~A}$ and a pulse width of $60 \mathrm{~s}$. Electrodes are gold-plated with platinum-iridium inner wire (negative contact) and stainless steel outer part (positive contact). The inner and outer electrodes are insulated except for a $75 \mu \mathrm{m}$ exposed tip (Tan et al., 2010).

Rats were divided in two groups, one group received implantation of electrodes in the $\mathrm{CIC}(\mathrm{n}=5)$ and the other group in the DCBN ( $n=5)$. In the off-stimulation state, designated as the control situation, no electrical stimulation was given. During stimulation-off state, LFS $(10 \mathrm{~Hz})$ and HFS $(100 \mathrm{~Hz})$, ABRs were recorded in separate sessions. 


\section{Auditory brainstem response}

ABR measurements were performed in a random manner of the three situations (off-stimulation, LFS, HFS) with a one week interval. Stimulation was turned on approximately 5 minutes before ABR recordings. HFS consisted of a concentric bipolar electrode using monophasic rectangular pulses, with a frequency of $100 \mathrm{~Hz}$, amplitude of $100 \mu \mathrm{A}$ per electrode and a pulse width of $60 \mu \mathrm{s}$ (A310 Acupulser, World Precision Instruments, Berlin, Germany). Similar settings were used in a study which showed tinnitus reduction during HFS in rats (Smit et al., 2016). LFS consisted of the same parameters with a frequency of $10 \mathrm{~Hz}$.

To achieve anesthesia during ABR recordings, intraperitoneal administration of ketamine $(90 \mathrm{mg} / \mathrm{kg})$ and xylazine $(10 \mathrm{mg} / \mathrm{kg})$ was used, which is preferred over isoflurane when assessing hearing thresholds in rats (Ruebhausen et al., 2012).

During the $A B R$ procedure, animals were placed into a sound-attenuating chamber. Cables were plugged into the socket of the head of the animal and connected to the recording device (Powerlab 8/35 connected to a Dual Bio Amp amplifier (ADInstruments, Castle Hill, Australia). An electrode connected to the left hind paw served as the ground.

Custrom-made auditory stimuli (10, 16, 24 and $32 \mathrm{kHz}$ ) were created with Matlab 2011a (Mathworks, MA, USA) and consisted of 5 ms bursts with a $\cos ^{2}$ rise and fall filter and were played at a rate of 20 per second at decreasing intensities from 90 to $0 \mathrm{~dB}$ peSPL with steps of $10 \mathrm{~dB}$. To prevent synchronous occurrence of stimulation artifacts with the ABRs, one in 10 stimuli had an interval of 55 ms instead of $45 \mathrm{~ms}$. To gain an approximately similar amount of data after filtering of stimulation artefacts, 500 auditory stimuli were given per intensity in the off-stimulation state, 700 during LFS and 1000 during HFS. Sounds were calibrated with a Bruel \& Kjaer 2231 decibel meter with a 4191 microphone (range $2-40 \mathrm{kHz}$ ), which was placed at the location of the rat's right ear. Sound intensities are reported as the peak equivalent sound pressure level (peSPL).

Auditory stimuli were processed with an external soundcard with a sample rate of $192 \mathrm{kHz}$ (Creative E-MU 0204), amplified with Ultrasonic power amplifier (Avisoft Bioacoustics, Berlin) and played with an Ultrasonic Dynamic Speaker Vifa (Avisoft Bioacoustics, Berlin, Germany) to the right ear. To standardize sound presentation between recording sessions it was monitored that in every session the same position of the rat and the same distance between the loudspeaker and the ear was used $(2 \mathrm{~cm})$. The contralateral ear was plugged with modeling clay.

Auditory stimuli were digitally triggered. The recordings were done in Labchart Pro 7 (ADInstruments, Castle Hill, Australia) at a sample frequency of $20 \mathrm{kHz}$ and raw data were imported into Matlab. With a customized script, the signal was amplified 100,000 times and band-pass filtered (300-3000 Hz). Evoked responses were averaged and data which contained DBS artifacts were automatically removed based on a peak-detection analysis. Using a customized Matlab script, peaks were automatically detected if the 
signal was above a manual depicted maximal baseline value. Before and after the maximal value of the peak of the artefact $2.5 \mathrm{~ms}$ of data were converted in Not-a-Number (NaN). The ABR and DBS stimuli were not phase-locked so per epoch a different part was converted in NaN. All epochs were averaged to calculate the mean ABR signal (B).
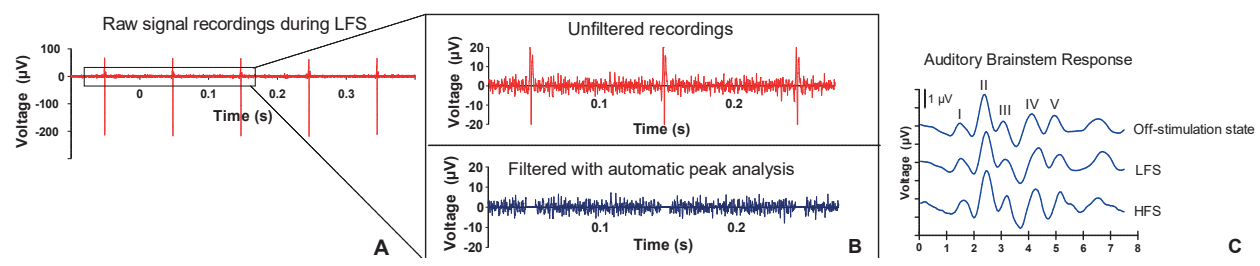

Figure 2. A. Example of a raw signal that was measured during low frequency stimulation (LFS). B. Stimulation artifacts are filtered with automatic peak detection analysis. C. Example of an auditory brainstem response (ABR) (burst frequency $10 \mathrm{kHz}$ ) during off-stimulation state, during LFS and during high frequency stimulation (HFS) in the central nucleus of the inferior colliculus (CIC). The five ABR peaks are numbered I-V. Morphology and latency of ABR peaks in the current study were consistent with other animal studies (Backoff and Caspary, 1994; Dehmel et al., 2012; Zheng et al., 2012). The first peak arises approximately 1.5 ms after stimulus onset. Although there is overlap, the first peak represents neural activity of the cochlear nerve. The second peak is considered to be mainly generated by cochlear nuclear cells, the third peak by the contralateral superior olivary complex cells and the fourth peak by the lateral lemniscus. The fifth peak, which appears approximately $5 \mathrm{~ms}$ after onset, originates from the IC (Biacabe et al., 2001; Chen and Chen, 1991; Simpson et al., 1985).

Two independent blinded observers visually identified ABR thresholds and peaks. In case of disagreement, a third observer was sought and the concordant data were accepted. The auditory threshold was defined as the lowest decibel level (peSPL) of the stimulus, which produced a distinctive ABR.

For latency analysis, the five positive peaks were determined at $90 \mathrm{~dB}$ peSPL and numbered I-V based on the recordings of vertex upward deflections (for an example see Figure $2 \mathrm{C}$ ). Latencies of peaks were measured from stimulus onset. Interpeak latency was defined as the time between respective peaks. The amplitude was expressed as the peak-to-peak amplitude ratio of peak $V$ subtracted by peak I.

\section{Electrode localization}

Animals were deeply anesthetized with pentobarbital $(75 \mathrm{mg} / \mathrm{kg}$ ) and perfused transcardially with Tyrode's buffer $(0.1 \mathrm{M})$ and fixative containing $4 \%$ paraformaldehyde, 15\% picric acid and $0.05 \%$ glutaraldehyde in $0.1 \mathrm{M}$ phosphate buffer $(\mathrm{pH} 7.6)$. After post-fixation for 12 hours, the brains were cut to coronal sections using a vibrotome. To assess the electrode localization, the sections containing the target area and the electrode trajectory were stained with hematoxylin-eosin (Merck, Darmstadt, Germany). Definition of anatomic structures was based on the stereotactical atlas (Paxinos and Watson, 2007). 


\section{Statistical analysis}

Dependent data were analyzed using the Wilcoxon signed-rank Test for two groups and a Friedman test for multiple groups. Since multiple comparisons were made when comparing the stimulation-off state with LFS and HFS, modified p-values (alpha $=0.05$ ) are given as corrected by means of the Holm-Bonferonni sequential correction (Holm, 1979). Data are presented as mean \pm standard error of the mean (SEM). All data were analyzed with SPSS (Version 20, IBM, Somers, NY, USA).

\section{Results}

\section{Electrode localization}

Histological evaluation showed that all electrodes were implanted correctly in the target structures (Figures $3 \mathrm{~A}$ and $3 \mathrm{~B}$, respectively).

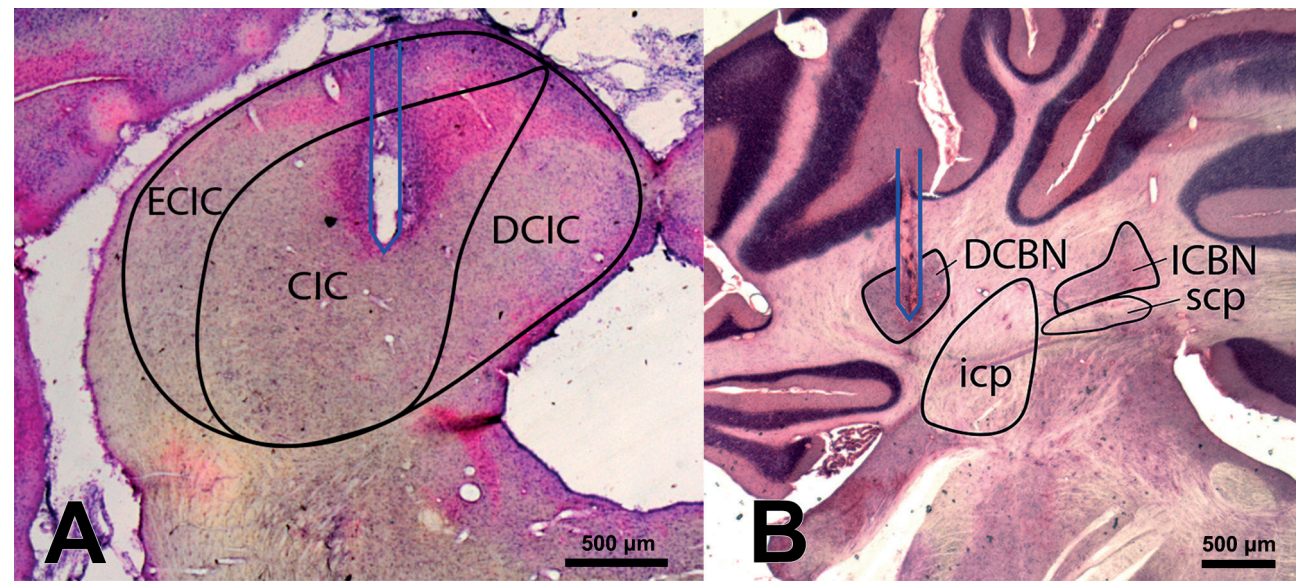

Figure 3: Representative examples of electrode positions (white lines) in the CIC (A) and DCBN (B). All electrodes were implanted bilaterally. $E C I C=$ external nucleus of the inferior colliculus, $C I C=$ central nucleus of inferior colliculus, $D C I C=$ dorsal cortex of inferior colliculus, $D C B N=$ dentate cerebellar nucleus, icp = inferior cerebellar peduncle, ICBN = interposed cerebellar nucleus, $s C p=s u p e r i o r$ cerebellar peduncle. Scale bar: $500 \mu \mathrm{m}$.

\section{Hearing thresholds}

Hearing response thresholds were determined as the minimal intensity stimulus at which an ABR was evident. Thresholds of different stimulus frequencies $(10,16,24$ and $32 \mathrm{kHz})$ are depicted in Figure 4A for the $\mathrm{CIC}$ group and in Figure 4B for the DCBN group. In one rat two thresholds ( $10 \mathrm{~Hz}$ LFS and $32 \mathrm{~Hz}$ LFS) were not possible to determine. In both groups, no statistically significant differences were found during HFS and LFS compared to off-stimulation. 
CNIC

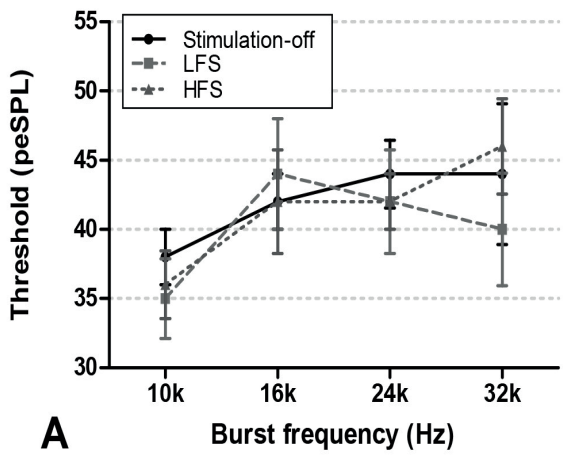

DCBN

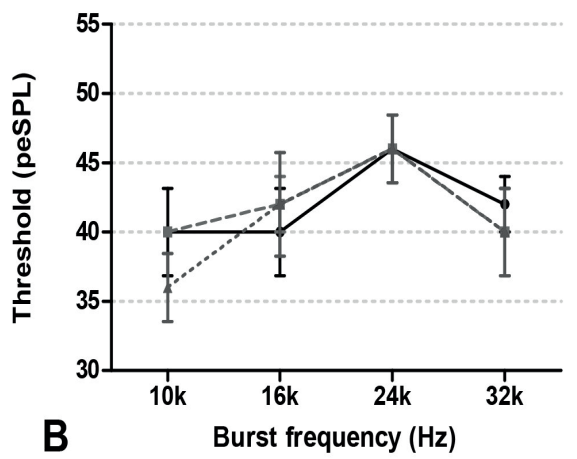

Figure 4. $A B R$ thresholds of the CIC (A) and DCBN group (B) measured during the DBS-off state (blue, circles, solid line), LFS (green, squares, striped line) and HFS (red, triangles, dotted line). There was no statistically significant difference. The vertical lines indicate the standard error of the mean.

\section{Latencies and amplitudes}

From all ABRs, 5 distinctive peaks could be determined at $90 \mathrm{~dB}$ peSPL (Figure 1). In Table 1 the mean interpeak latencies (I-III, III-V and I-V) are shown for different burst frequencies (10, 16, 24 and $32 \mathrm{kHz}$ ). In both the $\mathrm{CIC}$ and the DCBN group, no statistically significant differences were found for high and low frequency DBS compared to no stimulation (Table 1).

The V/I amplitude ratio was calculated at all burst frequencies. In both groups, there was no statistical significant difference when comparing no stimulation with HFS and LFS. Appendix 1 shows the absolute latencies and interpeak latencies.

When looking at the latency and amplitude data, a relation between ABR latencies and amplitudes, with frequencies of burst tones was noticed. For further analysis, we grouped the off-stimulation data of the $\mathrm{CIC}$ and DCBN group since only baseline measurements were analyzed. The latency, e.g. of peak I, differed between burst frequencies $\left(X^{2}(3)=20.12, p<0.01\right.$ ). The raw data (see Appendix 1 ) show a shorter latency with increasing frequencies of burst tones. The $\mathrm{V} / \mathrm{I}$ amplitude ratio does not differ amongst frequencies $\left(X^{2}(3)=4.92, p=.178\right)$. Amplitudes of peak I did not differ between frequencies $\left(X^{2}(3)=3.240, p=.355\right)$, but the amplitude of peak $\vee$ was different between frequencies $\left(X^{2}(3)=17.160\right.$, $p<0.01)$. Also peak $V$ amplitude decreases with increasing burst frequency. 


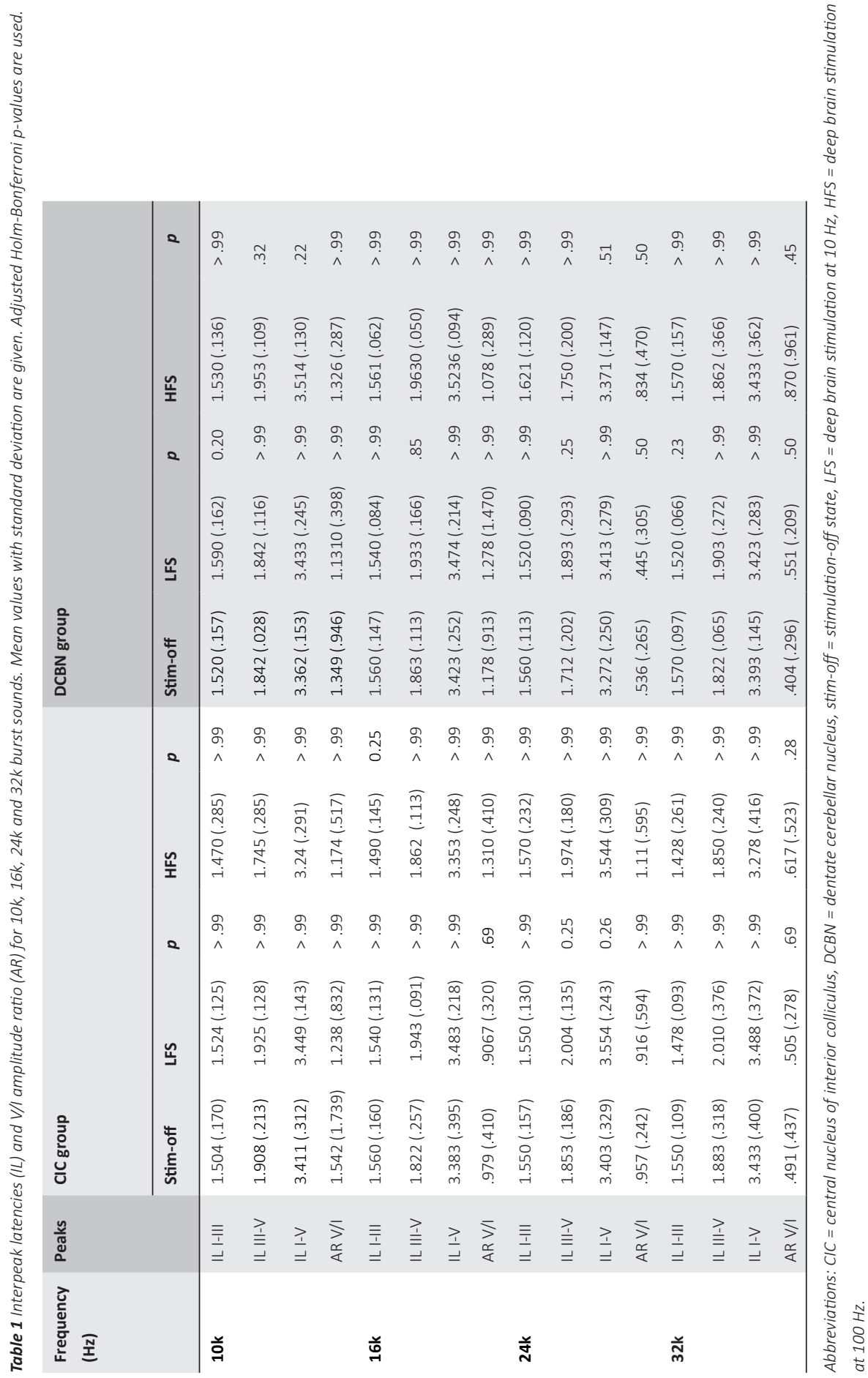




\section{Discussion}

We successfully measured ABRs during stimulation-off state, LFS and HFS. Our results showed that LFS as well as HFS in the CIC and DCBN do not influence ABR thresholds, interpeak latencies and amplitude ratios in rats.

\section{ABR thresholds}

The finding that ABR thresholds were not influenced by LFS and HFS suggests that hearing in these frequencies is not impaired by DBS. Nonetheless, several caveats must be taken into account when interpreting ABR thresholds. Although common frequencies were tested $(10,16,24$ and $32 \mathrm{kHz})$ in these studies, hearing loss can occur in other specific frequency bands. In rats, hearing thresholds based on ABRs tend to be at least 10-20 dB higher than those determined behaviorally (Borg, 1982, Heffner et al., 1994). The thresholds in the current study (ranging from 36 to $46 \mathrm{~dB}$ peSPL) are thus an overestimation of the actual hearing level. To get the most reproducible ABR data in various measurements, we implanted ABR electrodes. In contrast to the commonly used subcutaneous electrodes, these implanted electrodes always measure from exactly the same anatomical position (Buchwald et al., 1981, Hall, 1990, McGee et al., 1983). To our knowledge, no other studies determined ABR thresholds during HFS and LFS of the CIC or DCBN. Likewise, determination of thresholds in ablation studies, whose results are thought to be similar to HFS, have not been performed.

\section{ABR latency}

In addition to thresholds, the latency and amplitude can be extracted from the five ABR peaks. Interpeak latencies are generally accepted as measures of conduction time of the central auditory pathway (Eggermont and Don, 1986, Picton et al., 1977, Squires et al., 1978). The interpeak latency of waves $\mathrm{I}-\mathrm{III}, \mathrm{III}-\mathrm{V}$ and I-V reflect the time to traverse in the caudal, rostral and the whole brainstem, respectively. A prolonged interpeak latency reflects a lesion in central auditory processing (Burkhard et al., 2007, Hood, 1998). Occasionally, a decreased latency of peak $V$ was noted in ablation studies of the IC. This decrease of peak $V$ latency was only an acute effect (Achor and Starr, 1980).

In this study, no statistically significant differences were found between the interpeak latencies at baseline compared to low and high frequency DBS. This can be interpreted as no functional relevant lesion at the IC is induced by DBS. However, many studies found no differences in latencies when ablating the IC, but found a difference in amplitude (Achor and Starr, 1980, Buchwald and Huang, 1975, Caird and Klinke, 1987). Therefore, we also performed analysis of the ABR amplitude. 


\section{ABR Amplitude}

Synchronously activated neurons contribute to the amplitude of the waveform (Burkhard et al., 2007). The IC has a central role in the auditory pathway (Aitkin and Moore, 1975, De Martino et al., 2013). Previous studies have shown that lesioning of the IC resulted in a decrease of the amplitude of peak V (Achor and Starr, 1980, Buchwald and Huang, 1975, Caird and Klinke, 1987). In most studies a large part or the whole IC was ablated. One study only found an abolished peak $\mathrm{V}$ when ablation of the lateroventral part of the IC, in contrast to ablating the central nucleus (Funai and Funasaka, 1983). In humans, absence of the IC also resulted in abolished peak V peaks (Durrant et al., 1994). It is assumed that electrode implantation does not influence the amplitude of the evoked potentials, since only minimal tissue damage is seen along the electrode trajectory (Tan et al., 2010).

Although the precise role of the cerebellum and its associated nuclei in hearing is not known, it might have a modulatory effect on hearing. The cerebellum receives direct connections from the cochlear nucleus (Huang et al., 1982) and indirect connections from the IC (Aitkin and Boyd, 1978, Huffman and Henson, 1990). Furthermore, auditory stimuli as well as stimulation of the auditory cortex elicited responses from auditory cells in the paraflocculus (Azizi et al., 1985).

One study assessed the ABR during cerebellar stimulation. High frequency stimulation $(400 \mathrm{~Hz})$ of the cerebellar surface resulted in a difference of the IV/I amplitude ratio, where peak IV represented in this particular study the IC. The IV/I amplitude ratio increased in case of a short electrical-sound stimulus interval (<10 ms), and decreased with larger intervals (> $10 \mathrm{~ms})$. In this particular study, peak IV represented the IC (Crispino and Bullock, 1984). In our study, the electrical and sound stimuli were played in an asynchronous manner and therefore various interval times are achieved. This could explain why we did not found any difference in the amplitude ratio. As far as we know, no ABRs were recorded in a cerebellar ablation study.

\section{General ABR findings}

It is a well-known phenomenon that high frequency tones show shorter latency peaks than lower frequency sounds, because high frequency sounds stimulate the more basal portions of the basilar membrane (Alvarado et al., 2012). This is also seen in our data. We also found that the peak $V$ amplitude ratio decreased with increasing frequency of the tone given. As far as we know this is a new finding, which has not been reported earlier. 
Mechanism of DBS in the auditory system

Our results show that latencies were not prolonged and amplitudes were not decreased during DBS, indicating that DBS in the $\mathrm{CIC}$ and DCBN probably does not have an overall inhibitory effect on physiological central auditory processing up to the inferior colliculus (peak $\mathrm{V}$ ). This finding is supported by one of the main working mechanisms of DBS. Namely that DBS with frequencies above $100 \mathrm{~Hz}$ disrupts abnormal information flow in a network (Chiken and Nambu, 2014), without influencing the normal neurophysiological activity.

HFS is also often referred to as having an inhibitory effect and thus mimicking the effect of a lesion (Benabid et al., 1998, Dostrovsky and Lozano, 2002). The pathological neural network loop related to tinnitus is interrupted by performing HFS within this loop (Smit et al., 2016). This hypothesis is supported by the disruption theory; DBS can dissociate the input and output in a stimulation nucleus and thereby disrupting abnormal information flow such as increased burst activity. Physiological information can still be normally processed through different nuclei (Chiken and Nambu, 2014). It can be hypothesized that this is the same when DBS is applied in the auditory pathway and physiological auditory information processing remains intact.

\section{Future studies}

In the current study animal did not receive noise trauma for induction of tinnitus. We hypothesize that if DBS does not result in hearing loss in the normal hearing, this will also not be the case when there is hearing loss in association with tinnitus. The current stimulation parameters can be used for tinnitus treatment; in a recent study that showed a decrease of tinnitus during IC stimulation (Smit et al., 2016), the same stimulation parameters were used as in the current study. In our study no pre-operative assessment of the ABR was performed.

\section{Conclusions}

In conclusion, HFS and LFS in the CIC and DCBN did not result in increased ABR thresholds and changes in interpeak latencies. Based on these observations no evidence for changes in information processing in the auditory circuit were found during low and high frequency DBS in the CIC and DCBN. These findings suggest that DBS in the auditory pathways can be performed without hampering physiological processing of auditory information. 


\section{References}

Achor, L. J. \& Starr, A. 1980. Auditory brain stem responses in the cat. II. Effects of lesions. Electroencephalogr Clin Neurophysiol, 48, 174-90.

Aitkin, L. M. \& Boyd, J. 1978. Acoustic input to the lateral pontine nuclei. Hear Res, 1, 67-77.

Aitkin, L. M. \& Moore, D. R. 1975. Inferior colliculus. II. Development of tuning characteristics and tonotopic organization in central nucleus of the neonatal cat. J Neurophysiol, 38, 1208-16.

Alvarado, J. C., Fuentes-Santamaria, V., Jareno-Flores, T., Blanco, J. L. \& Juiz, J. M. 2012. Normal variations in the morphology of auditory brainstem response (ABR) waveforms: a study in Wistar rats. Neurosci Res, 73, 302-11.

Azizi, S. A., Burne, R. A. \& Woodward, D. J. 1985. The auditory corticopontocerebellar projection in the rat: inputs to the paraflocculus and midvermis. An anatomical and physiological study. Exp Brain Res, 59, 36-49.

Bauer, C. A., Kurt, W., Sybert, L. T. \& Brozoski, T. J. 2012. The cerebellum as a novel tinnitus generator. Hearing Research.

Bauer, C. A., Turner, J. G., Caspary, D. M., Myers, K. S. \& Brozoski, T. J. 2008. Tinnitus and inferior colliculus activity in chinchillas related to three distinct patterns of cochlear trauma. J Neurosci Res, 86, 2564-78.

Benabid, A. L., Benazzouz, A., Hoffmann, D., Limousin, P., Krack, P. \& Pollak, P. 1998. Long-term electrical inhibition of deep brain targets in movement disorders. Mov Disord, 13 Suppl 3, 119-25.

Borg, E. 1982. Auditory thresholds in rats of different age and strain. A behavioral and electrophysiological study. Hear Res, 8, 101-15.

Breit, S., Schulz, J. B. \& Benabid, A. L. 2004. Deep brain stimulation. Cell Tissue Res, 318, 275-88.

Brozoski, T. J., Ciobanu, L. \& Bauer, C. A. 2007. Central neural activity in rats with tinnitus evaluated with manganese-enhanced magnetic resonance imaging (MEMRI). Hear Res, 228, 168-79.

Buchwald, J. S., Hinman, C., Norman, R. J., Huang, C. M. \& Brown, K. A. 1981. Middle- and long-latency auditory evoked responses recorded from the vertex of normal and chronically lesioned cats. Brain Res, 205, 91-109.

Buchwald, J. S. \& Huang, C. 1975. Far-field acoustic response: origins in the cat. Science, 189, 382-4.

Burkhard, R. F., Eggermont, J. J. \& Don, M. 2007. Auditory evoked potentials: basic principles and clinical application, Philadelphia/ Baltimore, Lippincott/Williams \& Wilkins.

Caird, D. M. \& Klinke, R. 1987. The effect of inferior colliculus lesions on auditory evoked potentials. Electroencephalogr Clin Neurophysiol, 68, 237-40.

Chen, G. D. \& Jastreboff, P. J. 1995. Salicylate-induced abnormal activity in the inferior colliculus of rats. Hear Res, 82, 158-78.

Chiken, S. \& Nambu, A. 2014. Disrupting neuronal transmission: mechanism of DBS? Front Syst Neurosci, 8, 33. 
Crispino, L. \& Bullock, T. H. 1984. Cerebellum mediates modality-specific modulation of sensory responses of midbrain and forebrain in rat. Proceedings of the National Academy of Sciences of the United States of America, 81, 2917-20.

De Martino, F., Moerel, M., van de Moortele, P. F., Ugurbil, K., Goebel, R., Yacoub, E. \& Formisano, E. 2013. Spatial organization of frequency preference and selectivity in the human inferior colliculus. Nat Commun, 4, 1386

Dostrovsky, J. O. \& Lozano, A. M. 2002. Mechanisms of deep brain stimulation. Mov Disord, 17 Suppl 3, S63-8.

Durrant, J. D., Martin, W. H., Hirsch, B. \& Schwegler, J. 1994. 3CLT ABR analyses in a human subject with unilateral extirpation of the inferior colliculus. Hear Res, 72, 99-107.

Eggermont, J. J. \& Don, M. 1986. Mechanisms of central conduction time prolongation in brain-stem auditory evoked potentials. Arch Neurol, 43, 116-20.

Funai, H. \& Funasaka, S. 1983. Experimental study on the effect of inferior colliculus lesions upon auditory brain stem response. Audiology, 22, 9-19.

Gayer, N. S. \& Faull, R. L. 1988. Connections of the paraflocculus of the cerebellum with the superior colliculus in the rat brain. Brain Res, 449, 253-70.

Gould, B. B. 1979. The organization of afferents to the cerebellar cortex in the cat: projections from the deep cerebellar nuclei. J Comp Neurol, 184, 27-42.

Hall, R. D. 1990. Estimation of surviving spiral ganglion cells in the deaf rat using the electrically evoked auditory brainstem response. Hear Res, 49, 155-68.

Heffner, H. E., Heffner, R. S., Contos, C. \& Ott, T. 1994. Audiogram of the hooded Norway rat. Hear Res, 73, $244-7$.

Holm, S. 1979. A simple sequential rejective method procedure. Scandinavian Journal of Statistics, 6, 65-70.

Hood, L. J. 1998. Clinical applications of the Auditory Brainstem Response, San Diego, Singular Publishing Group.

Huang, C. M., Liu, G. \& Huang, R. 1982. Projections from the cochlear nucleus to the cerebellum. Brain Res, $244,1-8$.

Huffman, R. F. \& Henson, O. W., Jr. 1990. The descending auditory pathway and acousticomotor systems: connections with the inferior colliculus. Brain Res Brain Res Rev, 15, 295-323.

Kaga, K., Shinoda, Y. \& Suzuki, J. I. 1997. Origin of auditory brainstem responses in cats: whole brainstem mapping, and a lesion and HRP study of the inferior colliculus. Acta Otolaryngol, 117, 197-201.

Luo, H., Zhang, X., Nation, J., Pace, E., Lepczyk, L. \& Zhang, J. 2012. Tinnitus suppression by electrical stimulation of the rat dorsal cochlear nucleus. Neurosci Lett.

McGee, T. J., Ozdamar, O. \& Kraus, N. 1983. Auditory middle latency responses in the guinea pig. Am J Otolaryngol, 4, $116-22$.

Musiek, F. E., Kibbe, K., Rackliffe, L. \& Weider, D. J. 1984. The auditory brain stem response I-V amplitude ratio in normal, cochlear, and retrocochlear ears. Ear Hear, 5, 52-5. 
Musiek, F. E., Reeves, A. G. \& Baran, J. A. 1985. Release from central auditory competition in the split-brain patient. Neurology, 35, 983-7.

Osaki, Y., Nishimura, H., Takasawa, M., Imaizumi, M., Kawashima, T., Iwaki, T., Oku, N., Hashikawa, K., Doi, K., Nishimura, T., Hatazawa, J. \& Kubo, T. 2005. Neural mechanism of residual inhibition of tinnitus in cochlear implant users. Neuroreport, 16, 1625-8.

Paxinos, G. \& Watson, C. 2007. The Rat Brain in Stereotaxic Coordinates, London, Academic Press.

Picton, T. W., Woods, D. L., Barobeau-Brain, J. B. A. \& Healeu, T. M. G. 1977. Evoked Potential Audiometry. Journal of Otolaryngology, 6, 90-119.

Robertson, D., Bester, C., Vogler, D. \& Mulders, W. H. 2013. Spontaneous hyperactivity in the auditory midbrain: relationship to afferent input. Hear Res, 295, 124-9.

Rosahl, S. K., Tatagiba, M., Gharabaghi, A., Matthies, C. \& Samii, M. 2000. Acoustic evoked response following transection of the eighth nerve in the rat. Acta Neurochir (Wien), 142, 1037-45.

Ruebhausen, M. R., Brozoski, T. J. \& Bauer, C. A. 2012. A comparison of the effects of isoflurane and ketamine anesthesia on auditory brainstem response (ABR) thresholds in rats. Hear Res, 287, 25-9.

Sedley, W., Teki, S., Kumar, S., Barnes, G. R., Bamiou, D. E. \& Griffiths, T. D. 2012. Single-subject oscillatory gamma responses in tinnitus. Brain.

Shulman, A. \& Strashun, A. 1999. Descending auditory system/cerebellum/tinnitus. Int Tinnitus J, 5, 92-106.

Smit, J. V., Janssen, M. L., Schulze, H., Jahanshahi, A., Van Overbeeke, J. J., Temel, Y. \& Stokroos, R. J. 2015. Deep brain stimulation in tinnitus: current and future perspectives. Brain Res, 1608, 51-65.

Smit, J. V., Janssen, M. L., van Zwieten, G., Jahanshahi, A., Temel, Y. \& Stokroos, R. J. 2016. Deep brain stimulation of the inferior colliculus in the rodent suppresses tinnitus. Brain Res.

Squires, K. C., Chu, N. S. \& Starr, A. 1978. Auditory brain stem potentials with alcohol. Electroencephalogr Clin Neurophysiol, 45, 577-84.

Stockard, J. J. \& Rossiter, V. S. 1977. Clinical and pathologic correlates of brain stem auditory response abnormalities. Neurology, 27, 316-25.

Tan, S., Vlamings, R., Lim, L., Sesia, T., Janssen, M. L., Steinbusch, H. W., Visser-Vandewalle, V. \& Temel, Y. 2010. Experimental deep brain stimulation in animal models. Neurosurgery, 67, 1073-9; discussion1080.

Turner, J. G., Brozoski, T. J., Bauer, C. A., Parrish, J. L., Myers, K., Hughes, L. F. \& Caspary, D. M. 2006. Gap detection deficits in rats with tinnitus: a potential novel screening tool. Behav Neurosci, 120, 188-95.

Wang, J., Ding, D. \& Salvi, R. J. 2002. Functional reorganization in chinchilla inferior colliculus associated with chronic and acute cochlear damage. Hear Res, 168, 238-49. 


\section{Appendix: Absolute values of latencies and amplitudes}

Table 1: Absolute values of latencies and amplitudes from the five peaks of the auditory brainstem response of $10 \mathrm{kHz}$ auditory stimuli. Mean values with standard deviation are given.

\begin{tabular}{llllllll}
\hline Peak & Wave & \multicolumn{7}{l}{ CIC group } & \multicolumn{1}{l}{ DCBN group } \\
\cline { 3 - 8 } & & Stim-off & LFS & HFS & Stim-off & LFS & HFS \\
\hline Latencies & I & $1.639(.073)$ & $1.599(.110)$ & $1.618(.080)$ & $1.540(.165)$ & $1.490(.076)$ & $1.570(.090)$ \\
& II & $2.460(.092)$ & $2.479(.121)$ & $2.392(.079)$ & $2.386(.162)$ & $2.325(.066)$ & $2.356(.083)$ \\
& III & $3.143(.181)$ & $3.122(.208)$ & $3.088(.144)$ & $3.060(.280)$ & $3.080(.109)$ & $3.101(.084)$ \\
& IV & $4.288(.281)$ & $4.167(.191)$ & $4.122(.238)$ & $4.077(.260)$ & $4.118(.165)$ & $4.148(.098)$ \\
& V & $5.050(.275)$ & $5.047(.180)$ & $4.765(.291)$ & $4.903(.270)$ & $4.923(.206)$ & $4.983(.015)$ \\
& I & $.007(.018)$ & $.028(.030)$ & $.037(.042)$ & $.030(.016)$ & $.030(.011)$ & $.041(.047)$ \\
& II & $.221(.018)$ & $.218(.042)$ & $.252(.054)$ & $.159(.012)$ & $.181(.009)$ & $.185(.048)$ \\
& III & $.042(.018)$ & $.074(.037)$ & $.076(.038)$ & $.078(.031)$ & $.097(.066)$ & $.117(.069)$ \\
& IV & $.070(.017)$ & $.086(.042)$ & $.117(.035)$ & $.061(.055)$ & $.087(.041)$ & $.103(.053)$ \\
& V & $.030(.059)$ & $.054(.049)$ & $.063(.043)$ & $.077(.028)$ & $.069(.023)$ & $.093(.039)$ \\
\hline
\end{tabular}

Abbreviations: $C I C=$ central nucleus of interior colliculus, $D C B N=$ dentate cerebellar nucleus, stim-off = stimulation-off state, $L F S=$ deep brain stimulation at $10 \mathrm{~Hz}$, HFS = deep brain stimulation at $100 \mathrm{~Hz}$.

Table 2: Absolute values of latencies and amplitudes from the five peaks of the auditory brainstem response of $16 \mathrm{kHz}$ auditory stimuli. Mean values with standard deviation are given.

\begin{tabular}{|c|c|c|c|c|c|c|c|}
\hline \multirow[t]{2}{*}{ Peak } & \multirow[t]{2}{*}{ Wave } & \multicolumn{3}{|c|}{$\mathrm{CIC}$ group } & \multicolumn{3}{|c|}{ DCBN group } \\
\hline & & Stim-off & LFS & HFS & Stim-off & LFS & HFS \\
\hline \multirow[t]{5}{*}{ Latencies } & 1 & $1.400(.153)$ & $1.419(.042)$ & $1.530(.076)$ & $1.399(.042)$ & $1.389(.027)$ & $1.470(.066)$ \\
\hline & $\|$ & $2.245(.153)$ & $2.275(.055)$ & $2.325(.075)$ & $2.215(.128)$ & $2.285(.084)$ & $2.275(.065)$ \\
\hline & III & $2.960(.292)$ & $2.960(.125)$ & $3.020(.155)$ & $2.96(.140)$ & $2.929(.075)$ & $3.030(.090)$ \\
\hline & IV & $3.956(.367)$ & $4.067(.190)$ & $4.057(.230)$ & $3.977(.118)$ & $4.027(.155)$ & $4.098(.145)$ \\
\hline & V & $4.782(.534)$ & $4.903(.206)$ & $4.883(.249)$ & $4.822(.240)$ & $4.863(.213)$ & $4.993(.120)$ \\
\hline \multirow[t]{5}{*}{ Amplitudes } & 1 & $.042(.040)$ & $.039(.031)$ & $.037(.027)$ & $.036(.018)$ & $.025(.016)$ & $.058(.046)$ \\
\hline & $\|$ & $.182(.028)$ & $.162(.030)$ & $.189(.043)$ & $.139(.052)$ & $.119(.018)$ & $.178(.023)$ \\
\hline & III & $.079(.048)$ & $.080(.048)$ & .085 (.029) & $.088(.047)$ & $.079(.040)$ & $.118(.052)$ \\
\hline & IV & $.050(.019)$ & $.057(.031)$ & $.089(.029)$ & $.047(.015)$ & $.072(.030)$ & $.113(.035)$ \\
\hline & v & $.067(.047)$ & $.050(.028)$ & $.077(.029)$ & $.080(.089)$ & $.034(.023)$ & $.086(.040)$ \\
\hline
\end{tabular}

Abbreviations: $C I C=$ central nucleus of interior colliculus, $D C B N=$ dentate cerebellar nucleus, stim-off = stimulation-off state, LFS = deep brain stimulation at $10 \mathrm{~Hz}$, HFS = deep brain stimulation at $100 \mathrm{~Hz}$. 
Table 3: Absolute values of latencies and amplitudes from the five peaks of the auditory brainstem response of $24 \mathrm{kHz}$ auditory stimuli. Mean values with standard deviation are given.

\begin{tabular}{|c|c|c|c|c|c|c|c|}
\hline \multirow[t]{2}{*}{ Peak } & \multirow[t]{2}{*}{ Wave } & \multicolumn{3}{|c|}{$\mathrm{CIC}$ group } & \multicolumn{3}{|c|}{ DCBN group } \\
\hline & & Stim-off & LFS & HFS & Stim-off & LFS & HFS \\
\hline \multirow[t]{5}{*}{ Latencies } & 1 & $1.379(.126)$ & $1.379(.027)$ & $1.480(.131)$ & $1.299(.083)$ & $1.399(.075)$ & $1.419(.066)$ \\
\hline & II & $2.235(.200)$ & $2.215(.071)$ & $2.293(.111)$ & $2.134(.116)$ & $2.225(.083)$ & $2.305(.066)$ \\
\hline & III & $2.929(.234)$ & $2.929(.114)$ & $3.050(.131)$ & $2.859(.140)$ & $2.919(.094)$ & $3.040(.121)$ \\
\hline & IV & $3.896(.326)$ & $3.987(.186)$ & $4.097(.272)$ & $3.906(.170)$ & $4.007(.116)$ & $4.108(.166)$ \\
\hline & V & $4.782(.391)$ & $4.933(.228)$ & $5.023(.262)$ & $4.570(.215)$ & $4.812(.275)$ & $4.790(.129)$ \\
\hline \multirow[t]{5}{*}{ Amplitudes } & 1 & $.026(.025)$ & $.023(.038)$ & $.033(.027)$ & $.032(.011)$ & $.028(.014)$ & $.046(.045)$ \\
\hline & II & $.116(.020)$ & $.094(.020)$ & $.110(.028)$ & $.090(.016)$ & $.088(.015)$ & $.117(.029)$ \\
\hline & III & $.080(.062)$ & $.072(.062)$ & $.074(.031)$ & $.070(.033)$ & $.055(.034)$ & $.085(.072)$ \\
\hline & IV & $.037(.033)$ & $.192(.301)$ & $.083(.054)$ & $.053(.024)$ & $.054(.009)$ & $.077(.041)$ \\
\hline & V & $.034(.036)$ & $.033(.045)$ & $.043(.028)$ & $.022(.014)$ & $.013(.013)$ & $.050(.039)$ \\
\hline
\end{tabular}

Abbreviations: $C I C=$ central nucleus of interior colliculus, $D C B N=$ dentate cerebellar nucleus, stim-off = stimulation-off state, $L F S=$ deep brain stimulation at $10 \mathrm{~Hz}$, HFS = deep brain stimulation at $100 \mathrm{~Hz}$.

Table 4: Absolute values of latencies and amplitudes from the five peaks of the auditory brainstem response of $32 \mathrm{kHz}$ auditory stimuli. Mean values with standard deviation are given.

\begin{tabular}{|c|c|c|c|c|c|c|c|}
\hline \multirow[t]{2}{*}{ Peak } & \multirow[t]{2}{*}{ Wave } & \multicolumn{3}{|c|}{$\mathrm{CIC}$ group } & \multicolumn{3}{|c|}{ DCBN group } \\
\hline & & Stim-off & LFS & HFS & Stim-off & LFS & HFS \\
\hline \multirow[t]{5}{*}{ Latencies } & । & $1.369(.826)$ & $1.408(.051)$ & $1.497(.188)$ & $1.289(.104)$ & $1.268(.042)$ & $1.409(.155)$ \\
\hline & ॥ & $2.235(.145)$ & $2.222(.106)$ & $2.272(.122)$ & $2.104(.180)$ & $1.980(.444)$ & $2.325(.153)$ \\
\hline & III & 2.919 (.155) & $2.885(.124)$ & 2.926 (.139) & $2.859(1.80)$ & $2.789(.098)$ & 2.980 (.199) \\
\hline & IV & $3.946(.210)$ & $3.912(.130)$ & $3.982(.207)$ & $3.866(.232)$ & $4.097(.614)$ & $4.027(.216)$ \\
\hline & V & $4.802(.413)$ & $4.896(.371)$ & $4.776(.304)$ & $4.681(.222)$ & $4.691(.271)$ & $4.842(.277)$ \\
\hline \multirow[t]{5}{*}{ Amplitudes } & 1 & $.017(.011)$ & $.024(.032)$ & $.052(.124)$ & $.035(.017)$ & $.023(.013)$ & $.043(.041)$ \\
\hline & ॥ & $.097(.019)$ & $.156(.150)$ & $.167(.225)$ & $.078(.021)$ & $.091(.030)$ & $.092(.030)$ \\
\hline & III & $.062(.015)$ & $.093(.091)$ & $.134(.145)$ & $.058(.031)$ & $.056(.063)$ & $.058(.040)$ \\
\hline & IV & $.057(.048)$ & $.129(.141)$ & $.190(.268)$ & $.034(.026)$ & $.048(.028)$ & $.066(.042)$ \\
\hline & V & $.021(.022)$ & $.032(.054)$ & $.039(.090)$ & $.015(.146)$ & $.013(.025)$ & $0.042(.043)$ \\
\hline
\end{tabular}

Abbreviations: $C I C=$ central nucleus of interior colliculus, $D C B N=$ dentate cerebellar nucleus, stim-off = stimulation-off state, $L F S=$ deep brain stimulation at $10 \mathrm{~Hz}, \mathrm{HFS}=$ deep brain stimulation at $100 \mathrm{~Hz}$. 


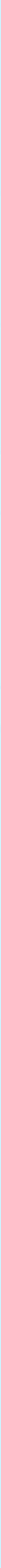




\section{Bhanter 7}

General discussion 


\section{Current concepts of tinnitus}

Despite the high prevalence of tinnitus, the burden on patients and the impact on society, successful treatment remains difficult. The main challenge in finding a treatment is the complex and not fully understood pathophysiology of tinnitus. Exploring the pathophysiology is challenged by the broad spectrum of tinnitus types and the current insufficient knowledge of auditory system physiology (de Ridder, 2011). Tinnitus research is repressed by the subjective nature of tinnitus, which makes it relatively unrecognized as an intractable and distressing condition. The limited funding that is allocated to tinnitus research is reflected in the small number of studies that are published in the field of tinnitus (Cederroth et al., 2013).

The development of new treatments requires thorough preclinical research studies, especially in case of invasive treatments. Animal models have contributed to our understanding of disorders as well as development of new therapeutic modalities. For instance, nearly all Nobel prizes in Medicine depended on animal research (The Foundation for Biomedical Research, 2017). Development of tinnitus animal models turned out to be important in gaining better understanding of the pathophysiology. Before animal models, the tinnitus mechanism was speculative for a long time and was restricted to the ear (Stephens, 1984). A crucial moment in exploring the mechanism was development of tinnitus animal models in the 1980's (Jastreboff et al., 1988), which led to the current concept of a central hypothesis (Kaltenbach, 2011). It is hypothesized that damage to the auditory periphery is usually the initial step in the origination of tinnitus. Due to homeostatic plasticity, neuronal hyperactivity is formed within the central nervous system (Norena, 2011, Schaette and Kempter, 2006). The first animal models were based on conditioned motor responses, and animals were trained to show different behavior in silence and in sound ('interrogative models'). After tinnitus induction, the sound-behavior was more prominent (Brozoski and Bauer, 2016). A new method is the gap-detection test using the acoustic startle reflex (Turner et al., 2006). This 'reflexive method' rapidly assesses tinnitus in a naive brain, making it easier to evaluate the effectiveness of new treatments. Various neuromodulatory treatments have been assessed with this method, such as vagus nerve stimulation (Engineer et al., 2011), cochlear nucleus stimulation (Luo et al., 2012) and cortical stimulation (Zhang et al., 2011) and drug studies have been performed using furosemide (Mulders et al., 2014), Ginkgo biloba (Tziridis et al., 2014) and cyclobenzaprine (Lobarinas et al., 2015).

The animal model for tinnitus is not entirely equivalent to human tinnitus, but allows experimentation such as finding the right stimulation target. Direct control over etiology of the disorder can be taken, such as choosing between salicylate-induced or noise-induced tinnitus. Furthermore, a single form of the disorder is studied whereas a human heterogeneous population only has some features in common (Brozoski and Bauer, 2016).

An example of successful preclinical work prior to clinical implantation is Parkinson's disease. Animal models formed the basis for treatment with deep brain stimulation (DBS) (Benazzouz et al., 1993) 
resulting in more than 150.000 Parkinson's disease patients being treated with DBS who were experiencing insufficient effects of pharmacological therapy (Wichmann and DeLong, 2016). There are several similarities between the pathophysiology of Parkinson's disease and tinnitus. In both diseases increased burst activity (Janssen et al., 2012, Kaltenbach et al., 2004) and impaired GABAergic neurotransmission has been demonstrated (Mallet et al., 2006, Dong et al., 2009). Interestingly, DBS reduces the amount of bursty subthalamic nucleus neurons in Parkinson's disease during stimulation (Hahn et al., 2008) and DBS could enhance GABA activity in output structures of the basal ganglia (Benazzouz and Hallett, 2000).

The exact mechanism behind the effects of DBS is unknown. A number of studies have led to the theory that DBS modulates local pathological activity and replaces it by a regular pattern of discharge with intervals of burst activity (rate and pattern hypothesis) (Birdno and Grill, 2008). A common finding is that a large amount of neurotransmitters is released by high-frequency electrical stimulation, such as GABA and glutamate. A supposed mechanism is that, as a result of the increase in inhibitory neurotransmitters, the input and output of the stimulated nucleus are dissociated and thereby disrupting the abnormal information flow (Ashkan et al., 2017, Lozano et al., 2002, Chiken and Nambu, 2016). However, it remains unclear how DBS exactly exerts a lesion-like effect. It can be hypothesized that the pathological neuronal activity that is seen in tinnitus is disrupted by DBS in the target area. As the auditory pathway consists of a number of interconnected structures, it is plausible that normal information flow could continue through collateral projections, preserving hearing. A similar process is seen when applying DBS in the basal ganglia/thalamocortical loop; electrical stimulation in healthy animals does not result in pathological motor activity (Hameleers et al., 2007, Tan et al., 2012, Badstuebner et al., 2017) but DBS in humans and animals with Parkinson's disease diminishes pathological movements (Temel et al., 2005, Benabid et al., 1987, Benazzouz et al., 1993).

\section{The effect of DBS on tinnitus}

In this thesis both human and animal studies were performed to assess the effect of DBS on tinnitus. The human study explored this effect in a retrospective setup; patients with implanted DBS electrodes for e.g. Parkinson's disease and essential tremor were questioned for presence of tinnitus before and after implantation. The rationale is that DBS may induce stimulated-related side-effects (Chan et al., 2009) and if tinnitus-related brain structures are (in)directly stimulated, there could be an influence on tinnitus. Taking into consideration the limitations, the questionnaire study was a relatively quick method to screen for the effect of DBS on tinnitus in various stimulated brain structures. This multicenter questionnaire study demonstrated a positive effect of DBS on tinnitus in Parkinson's disease patients who have electrodes implanted in the subthalamic nucleus. Despite that this brain region has no direct connection with tinnitus-related structures, it is connected to the nucleus accumbens, which has been shown to be involved in tinnitus (Rauschecker et al., 2010) and could therefore interfere 
with the pathological activity. An alternative hypothesis is that a perioperative focal lesion is induced in the caudate nucleus due to the traversing lead, which earlier has been shown to reduce tinnitus (Larson and Cheung, 2013). This finding led to a prospective study in which tinnitus was reduced during perioperative stimulation of the caudate nucleus (Cheung and Larson, 2010).

Even though beneficial effects of DBS in non-auditory structures have been found, subcortical auditory structures seem to be more closely involved in the core pathology of tinnitus (Kaltenbach, 2006, Norena, 2011). If DBS manages to interrupt the pathological activity in structures that are not directly linked to tinnitus, a stronger effect of DBS is predicted within the auditory pathway. The target of the experimental study in this thesis was a subcortical auditory structure; the external nucleus of the inferior colliculus. This target is involved in the auditory pathway, but also has indirect projections to and direct projections from the limbic system. The limbic system has been proposed to play an import role in the emotional reflection on tinnitus or by modulating tinnitus by acting as a selective gain-control mechanism (Jastreboff, 1990, Møller, 2011, Rauschecker et al., 2010). In this thesis, the external nucleus of the inferior colliculus structure was stimulated in an animal model for tinnitus. During high-frequency stimulation, tinnitus-like behavior was significantly reduced. As far as we know the effect of DBS in the inferior colliculus on tinnitus has not been studied before. The cochlear nucleus, however, has earlier been stimulated in an animal study and showed a positive effect on tinnitus (Luo et al., 2012).

In our animal tinnitus model, we used the prepulse inhibition of the acoustic startle response to assess presence of tinnitus. This relatively new model does not require pre-training, such as in interrogative animal models (Brozoski and Bauer, 2016). Therefore, neuroplastic changes that are directly related to tinnitus can be tested without confounding effects of previous conditioned paradigms. On the other hand, reflexive animal models mostly assess brainstem circuits, whereas interrogative models determine how an animal evaluates its tinnitus. Thus far, there is no preferred animal model, though for our research the prepulse inhibition method appeared to be more appropriate. In a within-subject experimental design, conditioned learning and motivational aspects can be influenced by surgery and tinnitus induction, which makes interrogative models not suitable. The chamber in which the rats were placed for assessing the startle reflex was customized to allow DBS during measuring the presence of tinnitus. Due to these adaptations the rats were able to freely move, which has the advantage of inducing less stress compared to fixated rats. The parameters of DBS were based on previous animal studies Parkinson's disease animal models (Temel et al., 2005). We hypothesized that the lesion-like effect of high-frequency stimulation was needed to interfere with the tinnitus-associated neural hyperactivity. Although, an earlier study has shown tinnitus reduction during low frequency stimulation $(10 \mathrm{~Hz})$ with similar current intensity (Luo et al., 2012). Though the results are promising, the results have to be interpreted with caution since no sham is used and the possible effect of hearing loss was not assessed. In contrast to our parameters, biphasic pulses were used with longer pulses (1000 $\mu \mathrm{s}$ versus $60 \mu \mathrm{s})$. In Parkinson's disease low frequency stimulation is ineffective and probably even exacerbates the symptoms (Rizzone et al., 2001). It has been hypothesized that during low-frequency stimulation neurons have time to return to normal activity in-between pulses. In contrast, during 
high-frequency stimulation the immediate effect of DBS summates with the late effect of the previous pulse producing a resonance effect (Montgomery and Gale, 2008). These findings and hypotheses are based on Parkinson's disease and therefore could not directly be applied to tinnitus. The supposed mechanism of low-frequency stimulation on tinnitus might be masking tinnitus signals. However, it is presumable that it also evokes side effects such as generation of sounds, because it increases activity within the auditory network. Based on these considerations, high-frequency stimulation seems to be more promising than low-frequency stimulation, but certainly the most optimal DBS parameters for tinnitus have yet to be determined.

Electrical stimulation within the pathological tinnitus network loop apparently results in interference with the pathological activity and thereby diminishes tinnitus. The optimal brain structure to be modulated with DBS needs to reduce tinnitus severity without inducing stimulation-related side-effects. For clinical applicability, a target structure has to be stereotactically accessible for DBS without damaging important brain structures. The inferior colliculus lies in the mesencephalon and has been reached with a craniotomy but has not yet been targeted for DBS in a clinical setting. We believe that DBS of auditory structures has more potential than stimulation of non-auditory structures. A relevant and easier-to-reach structure would therefore be medial geniculate body of the thalamus. The thalamus is frequently used for DBS, such as in essential tremor and experimentally in e.g. pain and epilepsy (Benabid et al., 1991, Pereira et al., 2012, Bittar et al., 2005). However the effectiveness of thalamic DBS for tinnitus has still to be determined.

\section{Potential side-effect of DBS for tinnitus}

High-frequency stimulation seems to have a similar effect as lesioning the target, as seen in Parkinson's disease (Benabid et al., 2002). One could argue that during high-frequency stimulation of auditory structures hearing loss can be induced. To investigate this potential side effect, hearing thresholds were assessed in this thesis with two different methods; sound-induced pre-pulse inhibition and auditory brainstem response. In this thesis, both techniques were applied in subsites of the inferior colliculus during DBS in animals that were not exposed to noise trauma. Both methods showed that DBS did not result in deterioration of hearing thresholds. Sound-induced pre-pulse inhibition is a method that indirectly assesses hearing by means of the acoustic startle reflex. The auditory role of the inferior colliculus within this test is exemplified in an animal study, which showed that lesioning the inferior colliculus resulted in abolishment of amplitude reduction to auditory prestimuli but not to visual prestimuli (Leitner and Cohen, 1985). The auditory brainstem response is more translatable from animal studies to patients, since the auditory brainstem response is also used in humans to assess hearing loss of retro cochlear origin (Stockard and Rossiter, 1977). The auditory brainstem response does not measure the whole auditory pathway since the latest peak reflects synchronous electrical activity of the inferior colliculus. 
Our findings suggest that high-frequency stimulation does not impair physiological activity within the auditory pathway. Despite that a lesion-like effect is mimicked with high-frequency stimulation, our findings fit in the disruption theory of DBS; only abnormal information is disrupted and thereby not interferes with physiological information (Chiken and Nambu, 2016). To our knowledge, no previous study assessed the effect of DBS on hearing. More potential side-effects of DBS in tinnitus-related structures, such as generation of sounds, could be investigated in future studies.

\section{The position of DBS in tinnitus treatment}

Apart from psychological intervention strategies, there are currently few therapies available for tinnitus. Hearing aids only have beneficial effect on tinnitus in the minority of patients (Kochkin and Tyler, 2008). Another form of hearing revalidation is cochlear implantation, which has shown to have a favorable effect on tinnitus in patients with bilateral or unilateral severe hearing loss (Arts et al., 2012, Ramakers et al., 2015). Cochlear implantation should however be preserved for patients with uni- or bilateral severe hearing loss because of surgical iatrogenic cochlear damage. An experimental invasive treatment for tinnitus is cortical stimulation by performing a craniotomy and placing a permanent electrode on the secondary auditory cortex. In a clinical study, 53\% reduction in tinnitus intensity was found (De Ridder et al., 2011). The study however, has several limitations; it was not placebo-controlled, inclusion and exclusion criteria were not shown, the effect on tinnitus burden and handicap were not assessed and the absolute values of the tinnitus intensity before and during stimulation were not shown. Complications involved epileptic seizures, intracranial bleeding and intracranial abscess. The precise role of cortical stimulation as a treatment for tinnitus remains to be evaluated. Non-invasive neuromodulation therapies, such as repetitive transcranial magnetic stimulation, have also been investigated for tinnitus, but there is insufficient evidence on long-term effectiveness (Hoekstra et al., 2013, Meng et al., 2011).

DBS has the advantage of being a minimal invasive surgery and can be reversed by not stimulating or by removing the electrode. Furthermore, DBS can target close to the source of the subcortical pathological tinnitus network loop. The position of DBS as a future treatment for tinnitus should be entitled to patients with sufficient residual hearing because patients with severe hearing loss are eligible for cochlear implantation. Candidates for DBS should be resistant to available treatments such as psychological treatments and hearing aids and should be motivated to have DBS treatment. The data presented in this thesis' survey study showed that around two fifth of the patients are reasonably to fully willing to undergo invasive therapies such as DBS. Patients with a history of attempted tinnitus treatment were more willing to undergo invasive treatments. Caution has to be taken not to rush through the informed consent since these patients tend to be desperate for these forms of invasive treatment which are often treatments of last resort (Grant et al., 2014). Balancing risk versus benefit is an important ethical consideration in DBS surgery (Schermer, 2011). This might be reflected in the finding that only slight increase in willingness was found when the chance of cure is $100 \%$ instead of 
$50 \%$. Therefore a complete informed consent has to be obtained with careful consideration of the treatment after weighing benefit and risks.

\section{Future perspective}

The results of this thesis form a scientific foundation for neuromodulative treatment for tinnitus using DBS. Further preclinical exploration should include assessment of DBS effects on the cochlear nucleus and the medial geniculate body of the thalamus. Both auditory structures are involved in the pathological network loop. The medial geniculate body of the thalamus has the advantage of having a better surgical accessibility than the more downstream auditory structures.

A prospective clinical study should be performed once sufficient preclinical data is obtained. Inclusion criteria for such a trial will include severe pure tone tinnitus which is chronic, stable and medically refractory. Patients should have hearing levels ranging from normal hearing to moderate hearing loss. The primary goal of such a study will be the effect of DBS on tinnitus, assessed by questionnaires. Secondary goals will be the effect of DBS on hearing, which can easily be assessed by audiometry, and the effect on neuropsychological outcomes such as quality of life. This practice can gain additional insight in the pathophysiology of auditory processing and tinnitus by means of neurophysiological measurements.

\section{Conclusion}

Taken together, neuromodulation within this pathological neuronal network is capable of reducing pathological activity and thereby diminishing tinnitus. Evidence was found in patients that were treated with DBS for another indication than tinnitus. This beneficial effect was found in patients that were stimulated in non-auditory regions, yet it is supposed that auditory regions are more superior targets for tinnitus because they are more closely involved in the tinnitus-related neural pathology. Based on a tinnitus animal model study, we found that DBS in the inferior colliculus was able to reduce tinnitus-like behavior. In animals no deterioration of hearing was seen during DBS.

Despite the risks of surgery, there is an acceptance of this treatment amongst a considerable number of tinnitus patients. Provided that an adequate ethical framework is used, DBS is a promising treatment for tinnitus patients with residual hearing who are refractory to current treatment. 


\section{References}

Arts, R. A., George, E. L., Stokroos, R. J. \& Vermeire, K. 2012. Review: cochlear implants as a treatment of tinnitus in single-sided deafness. Curr Opin Otolaryngol Head Neck Surg, 20, 398-403.

Ashkan, K., Rogers, P., Bergman, H. \& Ughratdar, I. 2017. Insights into the mechanisms of deep brain stimulation. Nat Rev Neurol, 13, 548-554.

Badstuebner, K., Gimsa, U., Weber, I., Tuchscherer, A. \& Gimsa, J. 2017. Deep Brain Stimulation of Hemiparkinsonian Rats with Unipolar and Bipolar Electrodes for up to 6 Weeks: Behavioral Testing of Freely Moving Animals. Parkinsons Dis, $2017,5693589$.

Benabid, A. L., Benazzous, A. \& Pollak, P. 2002. Mechanisms of deep brain stimulation. Mov Disord, 17 Suppl 3, S73-4.

Benabid, A. L., Pollak, P., Gervason, C., Hoffmann, D., Gao, D. M., Hommel, M., Perret, J. E. \& de Rougemont, J. 1991. Long-term suppression of tremor by chronic stimulation of the ventral intermediate thalamic nucleus. Lancet, 337, 403-6.

Benabid, A. L., Pollak, P., Louveau, A., Henry, S. \& de Rougemont, J. 1987. Combined (thalamotomy and stimulation) stereotactic surgery of the VIM thalamic nucleus for bilateral Parkinson disease. App/ Neurophysiol, 50, 344-6.

Benazzouz, A., Gross, C., Feger, J., Boraud, T. \& Bioulac, B. 1993. Reversal of rigidity and improvement in motor performance by subthalamic high-frequency stimulation in MPTP-treated monkeys. Eur J Neurosci, 5, 382-9.

Benazzouz, A. \& Hallett, M. 2000. Mechanism of action of deep brain stimulation. Neurology, 55, S13-6.

Birdno, M. J. \& Grill, W. M. 2008. Mechanisms of deep brain stimulation in movement disorders as revealed by changes in stimulus frequency. Neurotherapeutics, 5, 14-25.

Bittar, R. G., Kar-Purkayastha, I., Owen, S. L., Bear, R. E., Green, A., Wang, S. \& Aziz, T. Z. 2005. Deep brain stimulation for pain relief: a meta-analysis. J Clin Neurosci, 12, 515-9.

Brozoski, T. J. \& Bauer, C. A. 2016. Animal models of tinnitus. Hear Res, 338, 88-97.

Cederroth, C. R., Canlon, B. \& Langguth, B. 2013. Hearing loss and tinnitus--are funders and industry listening? Nat Biotechnol, 31, 972-4.

Chan, D. T., Zhu, X. L., Yeung, J. H., Mok, V. C., Wong, E., Lau, C., Wong, R., Lau, C. \& Poon, W. S. 2009. Complications of deep brain stimulation: a collective review. Asian J Surg, 32, 258-63.

Cheung, S. W. \& Larson, P. S. 2010. Tinnitus modulation by deep brain stimulation in locus of caudate neurons (area LC). Neuroscience, $169,1768-78$

Chiken, S. \& Nambu, A. 2016. Mechanism of Deep Brain Stimulation: Inhibition, Excitation, or Disruption? Neuroscientist, $22,313-22$. de Ridder, D. 2011. A Heuristic Pathophysiological Model of Tinnitus. In: MOLLER, A. (ed.) Textbook of Tinnitus.

De Ridder, D., Vanneste, S., Kovacs, S., Sunaert, S., Menovsky, T., van de Heyning, P. \& Moller, A. 2011. Transcranial magnetic 
stimulation and extradural electrodes implanted on secondary auditory cortex for tinnitus suppression. J Neurosurg, 114, 903-11.

Dong, S., Mulders, W. H., Rodger, J. \& Robertson, D. 2009. Changes in neuronal activity and gene expression in guinea-pig auditory brainstem after unilateral partial hearing loss. Neuroscience, 159, 1164-74.

Engineer, N. D., Riley, J. R., Seale, J. D., Vrana, W. A., Shetake, J. A., Sudanagunta, S. P., Borland, M. S. \& Kilgard, M. P. 2011. Reversing pathological neural activity using targeted plasticity. Nature, 470, 101-4.

Grant, R. A., Halpern, C. H., Baltuch, G. H., O’Reardon, J. P. \& Caplan, A. 2014. Ethical considerations in deep brain stimulation for psychiatric illness. J Clin Neurosci, 21, 1-5.

Hahn, P. J., Russo, G. S., Hashimoto, T., Miocinovic, S., Xu, W., McIntyre, C. C. \& Vitek, J. L. 2008. Pallidal burst activity during therapeutic deep brain stimulation. Exp Neurol, 211, 243-51.

Hameleers, R., Blokland, A., Steinbusch, H. W., Visser-Vandewalle, V. \& Temel, Y. 2007. Hypomobility after DOI administration can be reversed by subthalamic nucleus deep brain stimulation. Behav Brain Res, 185, 65-7.

Hoekstra, C. E., Versnel, H., Neggers, S. F., Niesten, M. E. \& van Zanten, G. A. 2013. Bilateral Low-Frequency Repetitive Transcranial Magnetic Stimulation of the Auditory Cortex in Tinnitus Patients Is Not Effective: A Randomised Controlled Trial. Audiol Neurootol, $18,362-373$.

Janssen, M. L., Zwartjes, D. G., Tan, S. K., Vlamings, R., Jahanshahi, A., Heida, T., Hoogland, G., Steinbusch, H. W., Visser-Vandewalle, V. \& Temel, Y. 2012. Mild dopaminergic lesions are accompanied by robust changes in subthalamic nucleus activity. Neurosci Lett, $508,101-5$.

Jastreboff, P. J. 1990. Phantom auditory perception (tinnitus): mechanisms of generation and perception. Neurosci Res, 8, 221-54.

Jastreboff, P. J., Brennan, J. F. \& Sasaki, C. T. 1988. An animal model for tinnitus. Laryngoscope, 98, 280-6.

Kaltenbach, J. A. 2006. Summary of evidence pointing to a role of the dorsal cochlear nucleus in the etiology of tinnitus. Acta oto-laryngologica. Supplementum, 20-6.

Kaltenbach, J. A. 2011. Tinnitus: Models and mechanisms. Hear Res, 276, 52-60.

Kaltenbach, J. A., Zacharek, M. A., Zhang, J. \& Frederick, S. 2004. Activity in the dorsal cochlear nucleus of hamsters previously tested for tinnitus following intense tone exposure. Neuroscience letters, 355, 121-5.

Kochkin, S. \& Tyler, R. S. 2008. Tinnitus treatment and the effectiveness of hearing aids: hearing care professional perceptions. Hearing Review, 15, 14-18.

Larson, P. S. \& Cheung, S. W. 2013. A stroke of silence: tinnitus suppression following placement of a deep brain stimulation electrode with infarction in area LC. J Neurosurg, 118, 192-4.

Leitner, D. S. \& Cohen, M. E. 1985. Role of the inferior colliculus in the inhibition of acoustic startle in the rat. Physiol Behav, 34, 65-70.

Lobarinas, E., Blair, C., Spankovich, C. \& Le Prell, C. 2015. Partial to complete suppression of unilateral noise-induced tinnitus in rats after cyclobenzaprine treatment. J Assoc Res Otolaryngol, 16, 263-72. 
Lozano, A. M., Dostrovsky, J., Chen, R. \& Ashby, P. 2002. Deep brain stimulation for Parkinson's disease: disrupting the disruption. Lancet Neurol, 1, 225-31.

Luo, H., Zhang, X., Nation, J., Pace, E., Lepczyk, L. \& Zhang, J. 2012. Tinnitus suppression by electrical stimulation of the rat dorsal cochlear nucleus. Neurosci Lett, 522, 16-20.

Mallet, N., Ballion, B., Le Moine, C. \& Gonon, F. 2006. Cortical inputs and GABA interneurons imbalance projection neurons in the striatum of parkinsonian rats. J Neurosci, 26, 3875-84.

Meng, Z., Liu, S., Zheng, Y. \& Phillips, J. S. 2011. Repetitive transcranial magnetic stimulation for tinnitus. Cochrane Database Syst Rev, CD007946.

Møller, A. R. 2011. Anatomy and Physiology of the Auditory System. Textbook of Tinnitus.

Montgomery, E. B., Jr. \& Gale, J. T. 2008. Mechanisms of action of deep brain stimulation(DBS). Neurosci Biobehav Rev, 32, $388-407$.

Mulders, W. H., Barry, K. M. \& Robertson, D. 2014. Effects of furosemide on cochlear neural activity, central hyperactivity and behavioural tinnitus after cochlear trauma in guinea pig. PLoS One, 9, e97948.

Norena, A. J. 2011. An integrative model of tinnitus based on a central gain controlling neural sensitivity. Neurosci Biobehav Rev, 35, 1089-109.

Pereira, E. A., Green, A. L., Stacey, R. J. \& Aziz, T. Z. 2012. Refractory epilepsy and deep brain stimulation. J Clin Neurosci, 19, 27-33.

Ramakers, G. G., van Zon, A., Stegeman, I. \& Grolman, W. 2015. The effect of cochlear implantation on tinnitus in patients with bilateral hearing loss: A systematic review. Laryngoscope, 125, 2584-92.

Rauschecker, J. P., Leaver, A. M. \& Muhlau, M. 2010. Tuning out the noise: limbic-auditory interactions in tinnitus. Neuron, 66, 819-26.

Rizzone, M., Lanotte, M., Bergamasco, B., Tavella, A., Torre, E., Faccani, G., Melcarne, A. \& Lopiano, L. 2001. Deep brain stimulation of the subthalamic nucleus in Parkinson's disease: effects of variation in stimulation parameters. J Neurol Neurosurg Psychiatry, 71, 215-9.

Schaette, R. \& Kempter, R. 2006. Development of tinnitus-related neuronal hyperactivity through homeostatic plasticity after hearing loss: a computational model. Eur J Neurosci, 23, 3124-38.

Schermer, M. 2011. Ethical issues in deep brain stimulation. Front Integr Neurosci, 5, 17.

Stephens, S. D. 1984. The treatment of tinnitus--a historical perspective. J Laryngol Otol, 98, 963-72.

Stockard, J. J. \& Rossiter, V. S. 1977. Clinical and pathologic correlates of brain stem auditory response abnormalities. Neurology, 27, 316-25.

Tan, S. K., Hartung, H., Visser-Vandewalle, V., Steinbusch, H. W., Temel, Y. \& Sharp, T. 2012. A combined in vivo neurochemical and electrophysiological analysis of the effect of high-frequency stimulation of the subthalamic nucleus on 5-HT transmission. Exp Neurol, 233, 145-53. 
Temel, Y., Visser-Vandewalle, V., Aendekerk, B., Rutten, B., Tan, S., Scholtissen, B., Schmitz, C., Blokland, A. \& Steinbusch, H. W. 2005. Acute and separate modulation of motor and cognitive performance in parkinsonian rats by bilateral stimulation of the subthalamic nucleus. Exp Neurol, 193, 43-52.

The Foundation for Biomedical Research, F. 2017. Nobel Prizes in Medicine [Online].

Turner, J. G., Brozoski, T. J., Bauer, C. A., Parrish, J. L., Myers, K., Hughes, L. F. \& Caspary, D. M. 2006. Gap detection deficits in rats with tinnitus: a potential novel screening tool. Behav Neurosci, 120, 188-95.

Tziridis, K., Korn, S., Ahlf, S. \& Schulze, H. 2014. Protective effects of Ginkgo biloba extract EGb 761 against noise trauma-induced hearing loss and tinnitus development. Neural Plast, 2014, 427298.

Wichmann, T. \& DeLong, M. R. 2016. Deep Brain Stimulation for Movement Disorders of Basal Ganglia Origin: Restoring Function or Functionality? Neurotherapeutics, 13, 264-83.

Zhang, J., Zhang, Y. \& Zhang, X. 2011. Auditory cortex electrical stimulation suppresses tinnitus in rats. J Assoc Res Otolaryngol, 12, 185-201. 


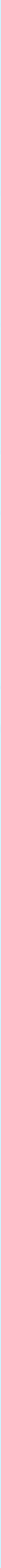




\section{Summary}

\section{Samenvatting}

Valorisation

Dankwoord

Biography 


\section{Summary}

In this thesis, the first step was to test our main hypothesis - tinnitus can be treated by deep brain stimulation (DBS)- by conducting a review and two questionnaire studies. The basis for this hypothesis is the current concept of the pathophysiology of tinnitus, which is discussed in the first section of the review (Chapter 2). According to this concept, cochlear damage leads to deafferentation of the eighth cranial nerve, which in turn exerts increased neuronal activity in the central nervous system. Several subcortical auditory and non-auditory regions show increased spontaneous activity, neuronal bursting and/or synchrony. In the review, potential auditory regions for DBS were chosen after analysis of literature; the cochlear nucleus, inferior colliculus and medial geniculate body of the thalamus. Potential non-auditory regions are the caudate nucleus, the ventral intermediate nucleus of the thalamus and the nucleus accumbens. One particular interesting region is the inferior colliculus; an important region in both the classical and non-classical auditory pathway, and directly and indirectly projected to the limbic system. The rationale behind the effect of DBS on tinnitus is mainly based upon the finding that DBS mimics a lesioning effect, thus decreasing hyperactivity in the pathological brain regions. Since it is unlikely that DBS only inhibits neuronal activity, the theory that DBS disrupts pathological activity seems to be more plausible.

DBS is already a clinical accepted treatment for Parkinson's disease and essential tremor. To find out whether DBS has any effect on tinnitus in the current targeted brain structures we performed a questionnaire study (Chapter 3). The most frequently targeted brain targets were the subthalamic nucleus, ventral intermediate nucleus of the thalamus and globus pallidus. In this study we compared outcomes of tinnitus questionnaires before and during DBS and we demonstrated that DBS has a favorable effect on tinnitus. The subthalamic nucleus was the only target that reached statistical significant difference. Despite these beneficial effects on tinnitus by stimulating non-auditory structures, stimulation of auditory structures which are more directly related to the core pathology of tinnitus seems to have more potential.

Despite that DBS is a minimally invasive surgery; there are associated risks, complications and costs. To assess whether patients are willing to undergo (minimally) invasive procedures such as DBS, we assessed a survey study among tinnitus patients (Chapter 4). The willingness of acceptance was assessed for hearing aids, cochlear implantation, cortical stimulation and DBS. We found that around one-fifth of the patients would be reasonably willing to accept invasive treatments and one-fifth would be fully willing to undergo invasive treatment. A weak correlation was found between acceptance of invasive treatments and tinnitus severity, and moderate correlations were found between acceptance of risks and tinnitus severity. Severe tinnitus sufferers seem to have higher acceptance towards invasive treatments; however, the associations are too weak to draw the conclusion that invasive treatments should only be offered to severe tinnitus sufferers. In addition we found that patients who already attempted one or more treatments were more eager to undergo an invasive therapy. In this light, it is important to realize that these forms of treatments are often a last-resort treatment, and with a 
comprehensive informed consent, risks and benefits have to be carefully weighted.

The second step in this thesis was to test our hypothesis with a tinnitus animal model (Chapter 5). The used animal model relies on the gap detection reflex procedures. Tinnitus-like behavior was demonstrated in 16 and $20 \mathrm{kHz}$ frequency bands, which is likely related to the induced $16 \mathrm{kHz}$ octave-banded hearing loss. We demonstrated that DBS in the external cortex of the inferior colliculus reduced behavioral signs of tinnitus. The most plausible side-effect of DBS in auditory structures is hearing loss. In the study, hearing thresholds of broadband noise were assessed with reflex procedures. We did not see any deterioration of hearing during DBS.

In Chapter 6 a more clinical relevant method of hearing assessment was measured with use of the auditory brainstem response during DBS in the central nucleus of the inferior colliculus. This nucleus is more primarily involved in auditory processing than the external nucleus of the inferior colliculus. It was demonstrated that the latency and amplitude of the auditory brainstem response did not change during DBS. Similar results were seen during DBS of the dentate cerebellar nucleus, a non-auditory structure related to tinnitus. These findings suggest that DBS in the auditory and non-auditory structures can be performed without hampering physiological processing of auditory information. 


\section{Samenvatting}

De eerste stap in dit proefschrift was om onze hypothese- tinnitus kan worden behandeld door diepe hersenstimulatie (deep brain stimulation; DBS) - te toetsen aan de hand van een literatuurstudie en twee vragenlijststudies. Deze hypothese is gebaseerd op de huidige opvatting over de pathofysiologie van tinnitus en wordt beschreven in een literatuurstudie (Hoofdstuk 2). Volgens deze hypothese leidt schade aan de cochlea tot deafferentiatie van de achtste hersenzenuw waardoor er een verhoging van de neuronale activiteit in het centraal zenuwstelsel optreedt. Verschillende auditieve en niet-auditieve hersengebieden laten verhoogde spontane activiteit, neuronale 'bursting' en/of synchroniteit zien. In de literatuurstudie worden de volgende potentiële auditieve hersengebieden beschreven; de nucleus cochlearis, colliculus inferior en corpus geniculatum mediale van de thalamus. Potentiële niet-auditieve gebieden zijn de nucleus caudatus, nucleus ventralis intermedius van de thalamus en de nucleus accumbens. Een bijzonder interessant gebied is de colliculus inferior; dit is een belangrijke structuur in zowel de klassieke als de niet-klassieke auditieve route en projecteert via directe en indirecte routes naar het limbische systeem. De achterliggende gedachte van het effect van DBS op tinnitus is gebaseerd op de bevinding dat DBS hetzelfde effect heeft als het aanbrengen van een laesie, namelijk het verminderen van hyperactiviteit in pathologische hersengebieden. Omdat het onwaarschijnlijk is dat DBS neurale activiteit alleen inhibiteert, lijkt de theorie dat DBS pathogische activiteit 'ontwricht' meer plausibel.

DBS wordt op dit moment in de kliniek toegepast voor patiënten met de ziekte van Parkinson en voor essentiële tremor. Er werd een vragenlijststudie verricht (Hoofdstuk 3) waarbij onderzocht werd of DBS in de huidige geïmplanteeerde hersenstructuren effect heeft op tinnitus. De meest gestimuleerde hersenkernen waren de nucleus subthalamicus, nucleus ventralis intermedius van de thalamus en globus pallidus. We vergeleken de uitkomsten van tinnitus vragenlijsten voor en tijdens DBS en we toonden aan dat DBS een positief effect heeft op tinnitus. De nucleus subthalamicus was het enige gebied waarbij de verbetering statistisch significant was. Ondanks dit positieve effect op tinnitus tijdens stimulatie van niet-auditieve gebieden, lijken auditieve gebieden- die directer gerelateerd zijn aan de bron van tinnitus- meer potentie te hebben.

Ondanks dat DBS minimaal invasive chirurgie is, zijn er geassocieerde risico's, complicaties en kosten. Om uit te zoeken of patiënten bereid zijn om (minimaal) invasieve therapieën zoals DBS te ondergaan, hebben wij een enquête gehouden onder tinnitus patiënten (Hoofdstuk 4). De bereidheid voor acceptatie werd onderzocht voor hoortoestellen, cochleaire implantatie, corticale stimulatie en DBS. Uit de enquête kwam naar voren dat ongeveer één vijfde van de patienten deels bereid was om invasieve behandelingen te ondergaan en één vijfde volledig bereid was om invasieve behandelingen te ondergaan. Een zwakke correlatie werd gevonden tussen acceptatie van invasieve behandelingen en de ernst van tinnitus, en een matige correlatie werden gevonden tussen acceptatie van risico's en de ernst van tinnitus. Patiënten met ernstig tinnitus lijken een hogere acceptatie te hebben voor invasieve behandelingen; deze associaties zijn echter te zwak om de conclusie te trekken dat invasieve 
behandelingen alleen aan patiënten met ernstig tinnitus mogen worden aangeboden. Daarnaast vonden we dat patiënten die al één of meerdere behandelingen hadden geprobeerd, meer belangstelling hadden om een invasieve therapie te ondergaan. Om deze reden is het belangrijk te beseffen dat deze vormen van behandelingen vaak een laatste behandelingsoptie zijn, en met een uitgebreide informed consent moeten de risico's en voordelen nauwkeurig worden afgewogen.

De tweede stap in dit proefschrift was het toetsen van onze hypothese aan de hand van een diermodel voor tinnitus (Hoofdstuk 5). Het gebruikte diermodel is gebaseerd op een gap-detectie reflex test. Gedrag passende bij tinnitus werd gezien in 16 en $20 \mathrm{kHz}$ frequentiebanden, wat waarschijnlijk gerelateerd is aan het geïnduceerde gehoorverlies in de $16 \mathrm{kHz}$ octaafband. We toonden aan dat tijdens DBS in de nucleus externus van de colliculus inferior minder gedragsmatige kenmerken passende bij tinnitus werden gezien. De meest plausibele bijwerking van DBS in auditieve gebieden is gehoorverlies. In deze studie werden gehoordrempels van breedbandruis gemeten met een reflex test. We zagen hierbij geen verslechtering van gehoor tijdens DBS.

In Hoofdstuk 6 werd een meer klinisch relevante methode van gehoorbepaling gemeten met de auditory brainstem response tijdens DBS in nucleus centralis van de colliculus inferior. Deze kern is primair meer betrokken bij de auditieve verwerking dan de nucleus externus van de colliculus inferior. Er werd aangetoond dat de latentietijd en amplitude van de auditory brainstem reponse niet veranderden tijdens DBS. Vergelijkbare resultaten werden gezien tijdens DBS van de nucleus dentatus, een niet-auditieve structuur gerelateerd aan tinnitus. Deze bevindingen suggereren dat DBS in de auditieve en niet-auditieve structuren kan worden uitgevoerd zonder dat de fysiologische verwerking van auditieve informatie wordt belemmerd. 


\section{Valorisation}

Tinnitus can pose a great burden on patients, their families and society. The impact on patients varies from a not-bothersome form (in $24 \%$ of the cases) to a form in which tinnitus plagues a patient all day long (17\%) (Axelsson and Ringdahl, 1989). This can lead to a series of psychological symptoms ranging from tension, frustration, sleep disturbance and loss of concentration, to depression and in severe cases even suicide (Andersson et al., 2005; Pridmore et al., 2012; Sullivan et al., 1988). It is not surprising that there is an impact on friends and family; $41 \%$ of the patients report negative effects on personal relationships (Holmes and Padgham, 2009).

Tinnitus-related societal costs in The Netherlands are estimated to add up to 6.8 billion euro per year. From these costs, health care costs of tinnitus were 1.9 billion euro, the rest is mainly related to productivity losses. The tinnitus-related health care costs account for $2.3 \%$ of the total Dutch health care expenditure (Maes et al., 2013).

At this moment, patients are not satisfied with the current tinnitus care; in The Netherlands a mean grade of 5.6 is given by patients. This grade is highest for patients with moderate symptoms (6.1) and is worse for patients with more severe symptoms (4.6). After the first outpatient visit, almost $75 \%$ of the patients consult a second doctor or other caregiver. Only 30\% of the patients reported to be benefited from this second visit (Schenk-Sandbergen, 2012). Outpatient visits mainly focuses on explanation, reassurance and education. Absence of a thorough treatment of tinnitus is likely to contribute to the struggle in tinnitus care.

The high impact of tinnitus on patients, society and economics on the one hand and the absence of an effective treatment on the other hand stresses the importance of developing new treatments for this condition.

Here, we investigated the effect of deep brain stimulation (DBS) on tinnitus to find a treatment for severe tinnitus sufferers. As DBS is an invasive procedure, finding patients willing to accept this treatment could be a first challenge. Therefore, we assessed the willingness of acceptance by means of a questionnaire study. Approximately $40 \%$ of the patients expressed that they were willing to undergo invasive neuromodulatory treatments such as DBS. Given the high prevalence of tinnitus, this figure can be considered substantial. Patients wish to undergo invasive neuromodulatory treatment despite a risk of sometimes severe side-effects, such as deafness or complications leading to death. For example, the risk of deafness and death were accepted as side effects by respectively $33 \%$ and $19 \%$ of the patients, when only a $50 \%$ tinnitus remission is expected. Based on these results and taken the high prevalence of tinnitus into account, a large group of patients could be treated by DBS. Regarding to ethical considerations in DBS, the risks and benefits have to be balanced thoroughly without hampering the patient's autonomy (Schermer, 2011). 
A quarter of the patients is willing to pay more than 20 times their monthly income for treatment if complete tinnitus elimination is given in prospect. In The Netherlands, this means a mean outlay of $€ 38,900$. At the moment, there is no reimbursement of outlay for these forms of invasive treatments for tinnitus since these treatments are still in state of development.

The outcomes of preclinical experiments in this thesis are in line with initial reports which have shown a positive effect of DBS on tinnitus. With preclinical studies valuable information regarding the optimal target for DBS in tinnitus can be extracted, which could lead to maximum therapeutic effects and cause minimal side effects. One of the targets to further be studied is the medial geniculate body of the thalamus. This target is also involved in the auditory pathway and is stereotactically easily reached in human DBS surgery.

Until now DBS has not been performed in auditory structures. A clinical study will provide a unique opportunity to record neuronal activity in central auditory structures. With the implanted DBS electrodes, local field potentials can be measured and can be combined with electroencephalography and auditory brainstem responses. These measurements lead to more insight in the auditory function such as central auditory crossing, tonotopic organization and allocation of attention to sound. This information is crucial to get a better understanding of altered activity in the auditory network. Finally, these recordings may establish a neurophysiological marker which could serve as an input parameter for a (closed-loop) neuromodulation device for individualized treatment.

In case of a future clinical study, multidisciplinary collaboration is essential for a successful project. Disciplines that need to be involved are departments of ear, nose and throat, neurosurgery, neurology, clinical neurophysiology, clinical psychology, neuropsychology and psychiatry. Because of the overlapping facets of tinnitus in these areas, future collaboration of disciplines is crucial. Therefore, this project could be a start in a more interdisciplinary approach in clinical tinnitus care. This may result in higher quality tinnitus care, with more satisfied patients.

Overall, this thesis is a step forward in finding an evidence-based treatment for tinnitus, which substantially diminishes the tinnitus without hampering physiological processes such as hearing. This therapy might not replace existing therapies but could serve as a treatment for therapy-resistant patients who highly suffer from tinnitus. The translational aspect of this thesis and the multidisciplinary environment paves the way for a clinical trial to assess tinnitus outcome during DBS. Secondary important, new information of central auditory processing will be gained. 


\section{References}

Andersson, G., et al., 2005. Tinnitus: a Multidisciplinary Approach, Vol., Whurr Publishers, London.

Axelsson, A., Ringdahl, A., 1989. Tinnitus- a study of its prevalence and characteristics. Br J Audiol. 23, 53-62.

Holmes, S., Padgham, N.D., 2009. Review paper: more than ringing in the ears: a review of tinnitus and its psychosocial impact. J Clin Nurs. 18, 2927-37.

Maes, I.H., et al., 2013. Tinnitus: a cost study. Ear Hear. 34, 508-14.

Pridmore, S., Walter, G., Friedland, P., 2012. Tinnitus and suicide: recent cases on the public record give cause for reconsideration. Otolaryngol Head Neck Surg. 147, 193-5.

Schenk-Sandbergen, L., 2012. Nieuwe geluiden over tinnitus en hyperacusis. Rapport tinnitus- en hyperacusis enquête Schermer, M., 2011. Ethical issues in deep brain stimulation. Front Integr Neurosci. 5, 17.

Sullivan, M.D., et al., 1988. Disabling tinnitus. Association with affective disorder. Gen Hosp Psychiatry. 10, $285-91$. 


\section{Dankwoord}

Het moment is daar, het boekje is af! Ik ben dankbaar voor iedereen die direct en indirect steun heeft gegeven. In het bijzonder wil ik de volgende personen bedanken:

Professor R.J. Stokroos, promotor, beste Robert, door jouw idee is dit project begonnen. Jouw daadkracht en visie in het wetenschappelijk onderzoek zorgden ervoor dat dit project opgezet kon worden. De samenwerking die je met de neurochirurgie hebt opgebouwd was hierbij essentieel. Heel erg bedankt!

Professor Y. Temel, promotor, beste Yasin, bedankt voor het opzetten van het project en voor je actieve betrokkenheid tijdens de gehele onderzoeksperiode. Ondanks je drukke klinische baan weet je zowel binnen als buiten werkuren altijd tijd vrij te maken voor overleg over onderzoek en hield je zicht op deadlines. Dank!

Dr. A. Jahanshahi, copromotor, "doctor Ali", jouw passie en toewijding voor onderzoek waren een grote toevoeging voor dit project. Je hebt me begeleid in het lab waar je alle ins en outs kent. Je vaardigheden gaan gepaard met grote precisie; ik ben dan ook blij dat ik van jou DBS operaties op ratten heb mogen leren. Naast het werk heb ik genoten van onze gezamelijke wielrentochten. Ik vlieg binnenkort graag eens met je mee in een Cessna vliegtuig!

Mark Janssen, je onuitputtelijke energie fascineert mij; zowel in het onderzoek als op de racefiets. Jouw tijdsinvesteringen in het project hebben mij steeds dichter bij mijn einddoel gebracht. Bedankt voor je actieve rol en oplossingsgerichte aanpak. Ik ben blij dat onze samenwerking blijft voortbestaan in het vervolgproject en ik kijk uit naar onze fietstochten.

Paranimfen, Marjolein Smit en Remo Arts. Zus, ik vind het erg speciaal dat je tijdens mijn promotie in Nederland kan zijn en ik ben vereerd dat je mijn paranimf wil zijn! Remo, ook naast onze onderzoekstijd hebben we een hele mooie tijd gehad, met als hoogtepunt het congres en aansluitend de reis door Amerika en Canada samen met onze vriendinnen. Bedankt allebei dat jullie mijn paranimfen willen zijn.

Gusta van Zwieten, ik ben blij dat jij het onderzoek hebt willen en kunnen doorzetten. De vele uren weggestopt in het lab waren even doorbijten (herkenbaar) maar hebben zeker zijn vruchten afgeworpen. Zonder jouw inzet was het project niet af en hadden we onvoldoende link naar een eerste prospectieve klinische studie. Heel veel succes met de laatste loodjes en je opleiding.

Mijn roomies en mede-promovendi, João ("Sjonnie") en Birgit, vanaf het begin zaten we letterlijk en figuurlijk in hetzelfde schuitje. Bedankt voor de gezellige tijd in de onderzoekskamer. Ook dank voor de andere promovendi van de neuroscience afdeling: Sarah, Lisa, Céline, Mélanie, Céline, Fred, Sandra, Özlem, Remco, Majed en de technici Denise en Helen. De mede-promovendi van de KNO en audiologie Marc, Elke en Anouk, ook bedankt voor de gezellige tijd. 
Professor Holger Schulze and Konstantin Tziridis, thank you for your hospitality in Erlangen. I really appreciated the time you invested in advising me how to set up the startle box. In addition thank you professor Schulze for your critic view on the review paper.

Alle overige co-auteurs wil ik graag bedanken voor hun bijdrage: Fiorella de Contarino, Arne Mosch, Rob de Bie, Richard Schuurman, Bart Pielkenrood, Koo van Overbeeke en Malou Engelhard. Miranda Janssen, bedankt voor je statistische hulp.

Alle stafleden en AIOS KNO. Bedankt voor de support, de mooie tijd en de mogelijkheden die gecreëerd zijn om naast de opleiding onderzoek te verrichten. Janny, bedankt dat op belangrijke momenten voor het onderzoek er de mogelijkheid was om de kostbare tijd die beschikbaar was in te plannen voor onderzoek.

Vrienden Pim, Thijs en Tijmen, dank voor jullie mooie momenten. De nu al legendarische weekendjes naar de meest schimmige bestemmingen op aarde hebben voor het nodige vertier gezorgd. Deze afleiding was en blijft onmisbaar. Tino, honnie, meer afleiding dan het slotconcert van Rowwen Hèze kan ik me niet voorstellen. Oud-leden van O.H.D. Bonobo, bedankt voor de mooie momenten zoals de lustrumreis. Op naar de volgende!

Uiteraard wil ik mijn familie graag bedanken, allereerst mijn vader en moeder. Heel erg bedankt voor de mogelijkheid die jullie mij hebben geboden om te doen wat ik doe. Ook wil ik mijn broer en zussen bedanken voor jullie gezelligheid en interesse.

Lieve Jeanny, ontzettend bedankt voor de steun die je mij hebt gegeven. Deze week maak je mij precies 10 jaar al heel gelukkig! Ik ben blij dat we mooie reizen samen hebben kunnen maken en nog blijer met de geboorte van onze kleine. Ik kijk uit om met jou de volgende uitdagingen aan te gaan! 


\section{Biography}

Jasper Vincent Smit was born on December 6th 1985 in Epe, Gelderland. After graduation from high school, he moved to Enschede to study the bachelor Biomedical Engineering at the University of Twente. This study combines science and biomedicine.

To concentrate towards the medical aspect of this field, he started the master Medical Engineering at the Eindhoven University of Technology. As study progressed, he became more and more interested in the medical field and found the perfect continuation of his study career; the four-year master study Medicine and Clinical Research at the Maastricht University.

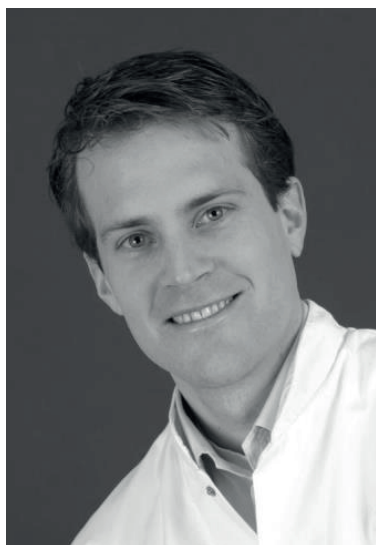

During the medical rotation at the Ear, Nose, and Throat department, his interest was raised in this specialty. Consequential, he decided to conduct research in tinnitus, which was initiated by professor R.J. Stokroos, otologist-neurotologist, and professor Y. Temel, neurosurgeon. This work formed the start of a two-year full time research period as a PhD-student in the Neuroscience Lab at the School for Mental Health and Neuroscience at the Maastricht University.

He initiated collaboration with professor $\mathrm{H}$. Schulze at the Experimental Otolaryngology Lab at the University of Erlangen-Nuremberg in Germany. He learned from him to build a startle box to assess tinnitus in rodents, which was fundamental to his research. Jasper presented his work at tinnitus congresses in Valencia (2013, Spain), Berlin (2014, Germany) and Ann Arbor (2015, United States).

At present Jasper works as a resident at the Ear, Nose and Throat, Head \& Neck Surgery Department at Maastricht UMC+. He recently started with his last-year specialization in otology and aims to finish his residency in 2019.

Jasper lives together with his girlfriend Jeanny Zuijlen. They are jubilant with the very recent birth of their son Sebastiaan. 
